\title{
Structural Design Considerations for Stretched- Membrane Heliostat Reflector Modules with Stability and Initial Imperfection Considerations
}

L. M. Murphy (SERI)

D. Simms (SERI)

D. V. Sallis (Dan-Ka Products)

October 1986

Prepared under Task No. 5111.31

FTP No. 510

Solar Energy Research Institute 


\section{NOTICE}

This report was prepared as an account of work sponsored by the United States Government. Neither the United States nor the United States Department of Energy, nor any of their employees, nor any of their contractors, subcontractors, or their employees, makes any warranty, expressed or implied, or assumes any legal liability or responsibility for the accuracy, completeness or usefulness of any information, apparatus. product or process disclosed. Or represents that its use would not infringe privately owned rights.

\section{Printed in the United States of America \\ Available from: \\ National Technical Information Service \\ U.S. Department of Commerce 5285 Port Royal Road \\ Springfield, VA 22161}

\section{Price: Microfiche A01 \\ Printed Copy A04}

Codes are used for pricing all publications. The code is determined by the number of pages in the publication. Information pertaining to the pricing codes can be found in the current issue of the following publications. which are generally available in most libraries: Energy Research Abstracts. (ERA): Government Reports Announcements and Index (GRA and I): Sclentific and Technical Abstract Reports (STAR); and publication, NTIS-PR-360 available from NTIS at the above address. 


\section{PREFACE}

The research and development described in this document was conducted within the U.S. Department of Energy's Solar Thermal Technology Program. The goal of this program is to advance the engineering and scientific understanding of solar thermal technology and to establish the technology base from which private industry can develop solar thermal power production options for introduction into the competitive energy market.

Solar thermal technology concentrates the solar flux using tracking mirrors or lenses onto a receiver, where the solar energy is absorbed as heat and converted into electricity or incorporated into products as process heat. The two primary solar thermal technologies, central receivers and distributed receivers, employ various point- and line-focus optics to concentrate sunlight. Current central receiver systems use fields of heliostats (two-axis tracking mirrors) to focus the sun's radiant energy onto a single, towermounted receiver. Point-focus concentrators up to 17 meters in diameter track the sun in two axes and use parabolic dish mirrors or Fresnel lenses to focus radiant energy onto a receiver. Troughs and bowls are line-focus tracking reflectors that concentrate sunlight onto receiver tubes along their focal lines. Concentrating collector modules can be used alone or in a multimodule system. The concentrated radiant energy absorbed by the solar thermal receiver is transported to the conversion process by a circulating working fluid. Receiver temperatures range from $100^{\circ} \mathrm{C}$ in low-temperature troughs to over $1500^{\circ} \mathrm{C}$ in dish and central receiver systems.

The Solar Thermal Technology Program is directing efforts to advance and improve each system concept through solar thermal materials, components, and subsystems research and development and by testing and evaluation. These efforts are carried out with the technical direction of DOE and its network of field laboratories that work with private industry. Together they have established a comprehensive, goal-directed program to improve performance and provide technically proven options for eventual incorporation into the nation's energy supply.

To successfully contribute to an adequate energy supply at reasonable cost, solar thermal energy must be economically competitive with a variety of other energy sources. The Solar Thermal Program has developed components and system-level performance targets as quantitative program goals. These targets are used in planning research and development activities, measuring progress, assessing alternative technology options, and developing optimal components. These targets will be pursued vigorously to ensure a successful program.

This report organizes and documents the numerous analysis findings relating to the predicted structural response and optical performance of stretched-membrane heliostat modules. The findings described herein evolved from the experimental and analytical activities carried out over the last two and one-half years and are built on work described in two prior SERI reports on the stretched-membrane heliostat concept. These activities also led to a current set of experiments on 3-m-diameter stretched-membrane modules to verify the analysis methods and predictions.

The author would like to acknowledge the contributions of Richard Wood, who provided numerous helpful discussions and insights through his work on the experimental program. 
This work was supported by the Division of Solar Thermal Technology of the U.S. Department of Energy. In particular, Martin Sheve and Frank Wilkins were very supportive in this effort, which is part of a larger overall initiative by DOE to identify and evolve innovative ways to achieve lower concentrator costs, while improving performance over today's technology of glass/metal-based designs.

The author would aiso like to acknowledge the pioneering work of others on the stretched-membrane heliostat concept. In particular, the DOE-funded work at the Boeing Co. with Roger Gillette et al. provided the early stretched-polymer-membrane heliostat concept for use with domed polymer enclosures in the late 1970s. The enclosed heliostat concept was later dropped from further consideration in the early 1980s, primarily when the analysis at SERI showed that considerable research progress needed to be made in various materials to achieve performance targets. Simultaneously, analysis also indicated that the concept of a stretched-metal membrane without an enclosure may be attractive. Thus interest in the unenclosed stretched-metal-membrane reflector was revived through the efforts of SERI and Sandia National Laboratories, Livermore (SNLL), in late 1980. Two individuals who played a significant role in reviving this interest were Dr. Barry Butler (while at SERI) and Tom Brumleve (while at SNLL).

In addition, we gratefully acknowledge the review comments provided by Dr. Barry Butler of Science Application International Corp. (SAIC) and Mr. Dave White of Solar Kinetics Inc.

Approved for

SOLAR ENERGY RESEARCH INSTITUTE

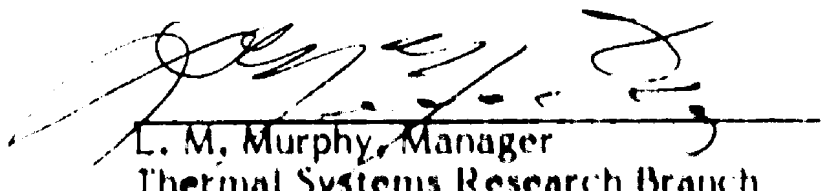

Therimal Systems Research Bramk

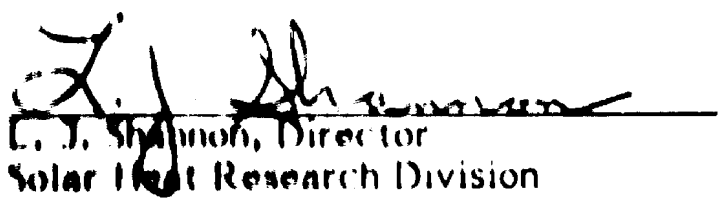




\section{SUMMARY}

\section{Objective}

The objective of this report is to present the results of numerous structural investigations that were carried out over the past two years on stretched-membrane heliostats. Most importantly this report provides insights on the trends and important design considerations that emerged from those investigations and that are important to the ultimate development of cost-effective stretched-membrane modules.

\section{$\underline{\text { Discussion }}$}

This report follows and extends the work of several previous reports that present the background leading to the development of stretched-membrane modules and analysis methods to study the structural response of the stretched-membrane module. Specifically this report presents and discusses the design implications based on our analysis of single- or double-membrane concepts, and the amplification of initial imperfections and deflections caused by loading, which results from stability considerations.

An understanding of the structural response of stretched-membrane modules is needed to design cost-effective and ultimately optimal stretched-membrane reflectors. Though simple in appearance, the structural response of the stretched-membrane module can be quite complex in its load/deformation response; this structure is not well studied in literature. The concept of the stretched-membrane heliostat reflector has been a DOE research focus for some time and is currently being guided through the development process by Sandia National Laboratories, Livermore,* with the help of industry partners. DOE supported the concept mainly because of the potential significant improvement in cost and performance, as discussed in previous works.

In this document we present analysis results for both single- and double-membrane concepts corresponding to a range of design and loading conditions. For idealized configurations and loadings, we discuss the relative merits of various design features for both of these designs. In addition, we studied the structural stability (i.e., the tendency of structural deformation to grow with little increase in applied load) of the tensionedmembrane, compressed-frame combination. Stability considerations are important in determining the amplification of both initial displacement imperfection and the deformations caused by wind and weight loading on the structure.

\section{Conclusions and Recommendations}

The analysis predictions carried out and described in this report have led to the following conclusions:

- Stretched-membrane/frame combinations respond quite differently to external loads than can be inferred by studying the decoupled frame and membrane independently. Thus the coupled membrane/frame problem should be considered to ensure an accurate description of its response.

\footnotetext{
* Responsibility for this development process has now been shifted to Sandia National Laboratories, Albuquerque.
} 
- Out-of-plane frame flexural rigidity is important for both single- and doublemembrane concepts. Frame torsional rigidity is not as important an issue with double-membrane concepts, but it becomes increasingly important when singlemembrane concepts are used and when low-stiffness membranes and/or lowstiffness attachments that connect the membrane to the frame are used.

- The method of attaching the membrane to the frame can affect the performance of the module significantly, especially with double-membrane designs, because the double membrane can enhance both the bending stiffness and the roll resistance of the frame.

- Stability considerations are important when considering the amplification of both initial imperfections and the amplification of the deformations that result from external loading by wind and weight environments. Compared to the frame alone, the membrane/frame combination, whether of the single- or double-membrane design, will have a greatly enhanced stability margin under radially directed loads. This is true for both in-plane and out-of-plane frame deformations. Moreover, double-membrane concepts increase the stability margin significantly above that for a single-membrane design. Further, the knowledge of the critical buckling tension level, which can be derived from the simple analyses presented herein, can result in some simple design tools.

- The double-membrane systems provide a very efficient structural system. From a weight perspective, the second membrane can, in fact, offer a more efficient structural stiffening effect than a corresponding increase in flexural rigidity with a single-membrane concept. This occurs since the double-membrane design couples the membrane stiffness into the problem in a more effective way than is possible with single-membrane designs. In addition, the second membrane adds significant stability margin to the reflector module and thus can inhibit the amplifications of initial imperfections as well as out-of-plane deformations caused by wind and weight loading.

The current analyses point to a number of design issues that may be critical to the design of optimal stretched-membrane modules, and we recommend that these issues be studied more thoroughly. More specifically, the following issues need the most immediate attention: the flutter-induced fatigue of the membrane; the potential to unload the membranes in double-membrane designs as the frame twists and moves radially inward; the effects of nonuniform wind-induced pressure loading; the effects of in-plane weight loading; and finally the effects caused by supports that have finite stiffness. 


\section{TABLE OF CONTENTS}

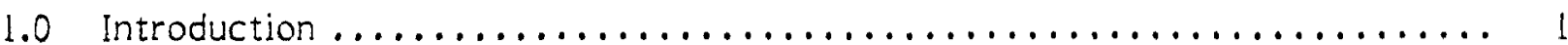

2.0 Response Trends for Single- and Double-Membrane Modules ............ 4

2.1 Some General Load Deformation Response Characteristics ........... 4

2.2 Frame Bending Stiffness Effects ...................... 8

2.3 Response Trends Caused by Material Selection ................ 12

2.4 Frame Torsional Stiffness Effects ....................... 15

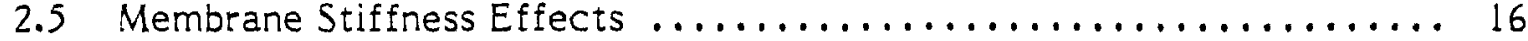

2.6 Effects Caused by Changes in Frame Radius and Number of

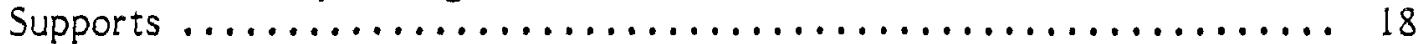

2.7 Some Thoughts on In-Plane Deformations ................. 20

2.8 A Potential Problem with Double-Membrane Modules ............. 21

3.0 Stability Considerations $\ldots \ldots \ldots \ldots \ldots \ldots \ldots \ldots \ldots \ldots \ldots \ldots \ldots \ldots \ldots \ldots \ldots$

3.1 General Considerations ............................ 22

3.2 Stability Considerations for Single-Membrane Concepts ........... 24

3.3 Stability Considerations for Double-Membrane Concepts.............. 30

3.4 Stability and the Impact on Initial Imperfections ............... 31

4.0 Design Implications and Recommended Areas for Further

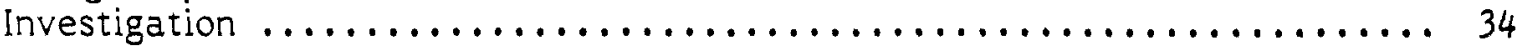

4.1 Implications for the Design of Stretched-Membrane Modules . . . . . . . . 34

4.2 Structural Response Issues Requiring Further Investigation ......... 36

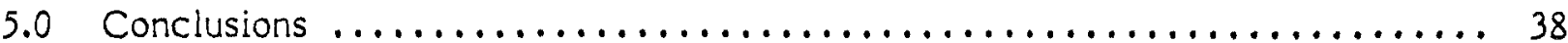

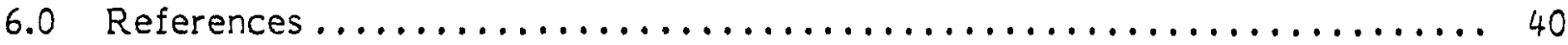

Appendix A An Eigenvalue Approach to Determining the Critical Bifurcation Tension Load for a Single-Membrane Module Design............. 42

Appendix B A Nonlinear Axisymmetric Membrane/Support Frame Load

Deformation Relationship ........................ 46

Appendix C In-Plane Buckling of Ring/Membrane Combination ........... 50

Appendix D Amplification Factors for Initial Imperfections and

Lateral Loading Corresponding to Single-Membrane Designs........ 53 


\section{LIST OF FIGURES}

$\underline{\text { Page }}$

1-1 Typical Stretched-Membrane Module Geometry Including

Single-/Double-Membrane Frame Cross Sections Considered

in Numerous Examples Discussed in this Report

2-1 Axisymmetric and Asymmetric Deformation Modes for a

Typical Membrane .................................

2-2 Maximum Frame Displacement Midway between the Supports as a

Function of the Total Effective Load per Unit Reflector Area .......... 5

2-3 Membrane Attachment Details Showing Radial and Hard Attachments ..... 6

2-4 Effective Lateral Stiffness Ratio versus Total Membrane Tension on the Frame for Three Module Designs .................... 7

2-5 Flexural and Torsional Rigidity of Frame as a Function of

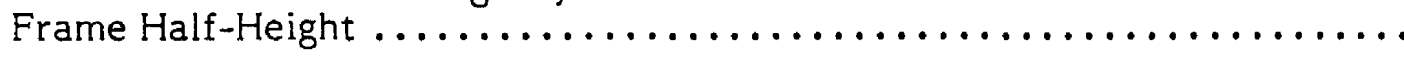

2-6 Gravity and Wind-Induced Loading Assumed for the Modules

Considered in Figures 2-7 through 2-10

2-7 Peak Lateral Frame Deformation (Midway between the Supports) as a Function of Frame Half-Height for Single-Membrane, DoubleMembrane (with Radial Attachment), and Double-Membrane (with Hard Attachments) Module Designs ......................... 10

2-8 Peak Membrane Slope Error Corresponding to the Asymmetric Deformations as a Function of Frame Half-Height for Three Module Designs Considered in Figure $2-7 \ldots \ldots \ldots \ldots \ldots \ldots \ldots \ldots \ldots \ldots$

2-9 Membrane rms Surface Error (Asymmetric Portion) as a Function of Frame Half-Height for the Three Module Designs Considered in Figures $2-7$ and $2-8 \ldots \ldots \ldots \ldots \ldots \ldots \ldots \ldots \ldots \ldots \ldots \ldots \ldots \ldots \ldots$

2-10 Membrane rms Surface Error (Asymmetric Portion) as a Function of Weight per Unit Reflector Area for the Three Module Designs Considered in Figures $2-7$ through $2-9 \ldots \ldots \ldots \ldots \ldots \ldots \ldots \ldots \ldots \ldots$

2-11 Peak Lateral Frame Deformation (Midway between the Supports) as a Function of Frame Half-Height for SiC/Al, Aluminum, and Steel Double-Membrane Designs ........................

2-12 Membrane rms Surface Error (Asymmetric Portion) as a Function of Frame Half-Height for SiC/A1, Aluminum, and Steel Double-Membrane

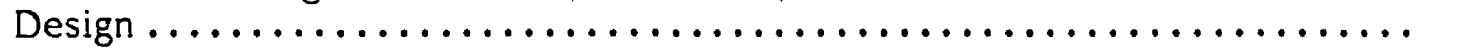




\section{LIST OF FIGURES (continued)}

2-13 Membrane rms Surface Error (Asymmetric Portion) as a Function of Total Weight per Unit Reflector Area for SiC/A 1 , Aluminum, and Steel Double-Membrane Design ......................... 14

2-14 Maximum Vertical Displacement of Frame (Midway between Supports) as a Function of Out-of-Plane Frame Bending Rigidity (EI) for Single- and Double-Membrane (with Radial Attachments) Designs at a Constant Value of Torsional Rigidity ........................

2-15 Maximum Vertical Displacement of Frame (Midway between Supports) as a Function of Frame Torsional Rigidity (GK), for Single- and Double-Membrane (with Radial Attachments) Designs at a Constant Value of Frame Bending Rigidity

2-16 Stretched-Membrane Performance as a Function of Frame HalfHeight for a Single-Membrane and Two Double-Membrane Designs with Lowered Membrane Moduli ........................

2-17 Effective Lateral Module Stiffness as a Function of Membrane Modulus for Single- and Double-Membrane (with Hard Attachments) Designs .......................................

2-18 Maximum Frame Deflection and Rotation versus the Number of Supports .......................................

2-19 Areal Weight versus the Number of Supports for Several Prescribed rms Surface Error Levels ..........................

2-20 Maximum Frame Deflection as a Function of Frame Radius for Designs Using Either Three, Four, or Five Evenly Spaced Simple Rigid Supports . . . . . . . . . . . . . . . . . . . . . . . . .

3-1 Linear Southwell Plot for a Simple Beam Structure . . . . . . . . . . . . . . . .

3-2 Geometry and Loading on the Ring Frame with and without the Membrane Attached .................................

3-3 Peak Out-of-Plane Frame Deflection versus Radial Tension Load for a Number of Frame/Single-Membrane Combinations ............

3-4 Single-Membrane Deformation versus Radius at Several Circumferential Sections Corresponding to the $n=2$ Frame Deformation Mode ..................................

3-5 Peak Out-of-Plane Deflection as a Function of Tension for Several Single- and Double-Membrane Designs Corresponding to the $\mathrm{n}=2$ Frame Deformation Mode 


\section{LIST OF FIGURES (concluded)}

3-6 Out-of-Plane Deflection Comparison versus Tension for a Manufacturing Type (Fixed), $\mathrm{n}=2$ Mode, Initial Frame

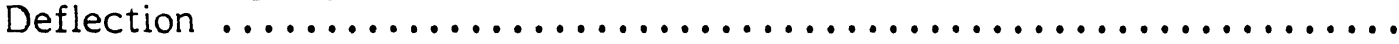

A-1 Frame and Cross-Section Detail Showing Displacements and the Corresponding Directions and Applied Loading ............... 42

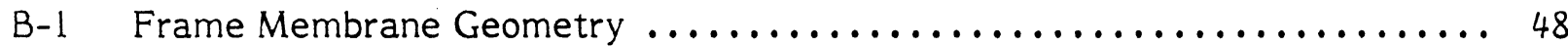

B-2 Membrane Center Deformation Ratio and Fractional Tension Increase as a Function of Initial Membrane Tension for Several

Pressure Loadings and Support Ring Stiffnesses ................ 4

D-1 Ring Lateral Deformation Amplification Factor versus Operating/

Critical Tension Ratio $(n=2)$

D-2 Predicted Maximum Frame Displacement Midway between the Supports as a Function of Tension Ratio for Two Lateral Pressure Loading Conditions

\section{LIST OF TABLES}

2-1 Material Properties Assumed in Figures $2-11$ through $2-13 \ldots \ldots \ldots \ldots \ldots$

A-1 Critical Membrane Tension Loads for Several Geometric Configurations ................................... 45

C-1 Critical In-Plane Buckling Parameters for the Ring/Membrane

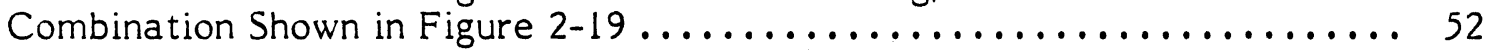

D-1 Amplification of Mode Shapes .......................... 55 


\subsection{INTRODUCTION}

The emphasis of this report is on extending our knowledge of the optical and structural response of the stretched-membrane concept to help accelerate the development and design of cost-effective heliostat reflector modules. The stretched-membrane heliostat concept, its importance, and the context in which it would appear to have the most impact, are discussed in Murphy (1983). Ultimately we hope that this work will assist in developing design guidelines and requirements for cost-effective reflector modules when coupled with the development work that DOE is now carrying out under Sandia National Laboratories, Livermore (SNLL).

In a previous report, we developed and used a methodology to aid in the understanding of various response mechanisms (Murphy and Sallis 1984) that affect the optical and structural performance and, ultimately, the design and cost of single-membrane modules. In another report (Murphy 1985), we describe a more comprehensive analytical method to consider a wider range of design issues. In Murphy (1985), the capability to study double-membrane modules and the attendant stiffening benefit that can accrue is provided along with the ability to analyze the effect of various attachment approaches on performance. In this report, we study these issues in relation to designing for optimal performance (not cost) in stretched-membrane modules. Further, in this report we introduce stability considerations and investigate the resulting implication to both the design and performance of the reflector module. We use the method in Murphy (1985) as well as the NASTRAN finite element code (Schaeffer 1979) for the performance predictions presented here.

The study of the double-membrane concept, which can be described as a drumlike structure formed by a support frame on which two parallel membranes are stretched (see Figure 1-1), is important from a number of perspectives. Such modules are currently being developed because of their good adaptability to focusing; focusing can be accomplished by partially evacuating the plenum chamber between the membranes, thus causing the reflective membrane to curve and hence focus. Moreover, the doublemembrane concept offers several significant structural performance benefits that may outweigh the benefits of single-membrane designs in cases where the vacuum focusing is not needed.

Attachments are important considerations not only from a practical production perspective but also because the method of attachment can influence the level at which the membrane stiffness can be coupled into the frame. This is especially true with the double-membrane designs. The coupling effect can enhance either the bending or rollresistance capability of the frame or both in retarding out-of-plane deformations and can improve the structural performance of the module significantly. Further, this coupling effect can influence the degree of stability enhancement that the membranes can offer to the system.

Stability considerations are also of concern even though typical early designs will be conservative; membrane tensions will be well below the critical levels, and collapse of the structure will not be of concern. These stability considerations are important because the nearness to the critical tension level determines the level at which the amplifications of initial imperfections and of lateral deformations caused by wind and weight loading will occur. Hence researchers will ultimately need this information to fully evaluate the optical quality of the reflective surface and to develop optimal structures. Further, the knowledge of the critical tension levels allows for a more 


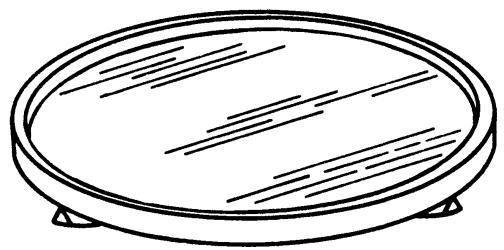

(a) Perspective view - stretched-membrane reflective module; pin supported at three equidistant clrcumferential points

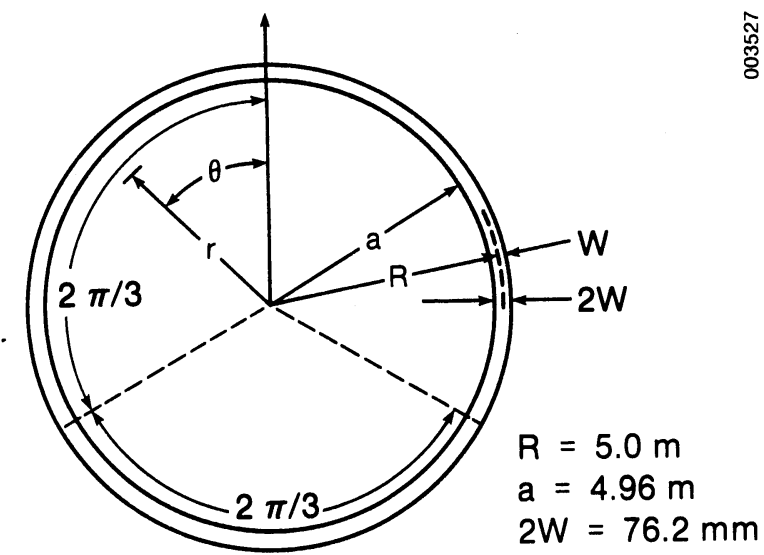

(b) Top view - stretched-membrane reflectlve module

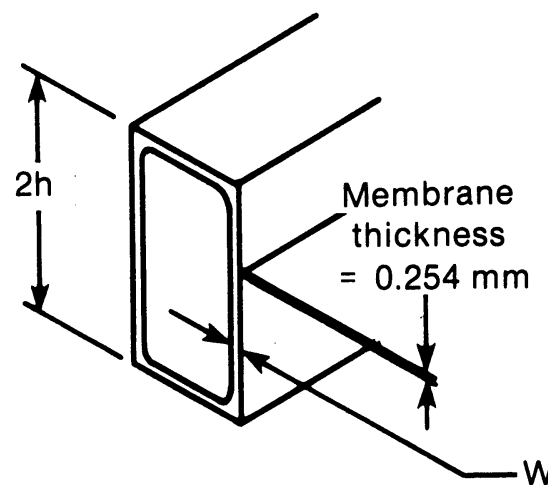

Wall thickness

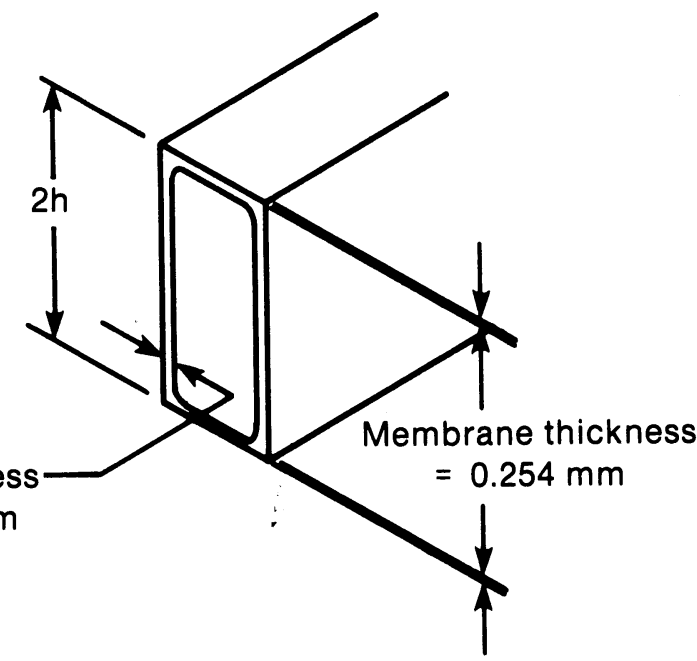

(c) Cross-section view for steel frame/membrane combination considered in analysis discussion

Figure 1-1. Typical Stretched-Membrane Module Geometry Including Single-/ Double-Membrane Frame Cross Sections Considered in Numerous Examples Discussed in this Report 
complete understanding of the deformation process and can also lead to the development of simple design tools.

Note that the idealized frame and membrane geometry as illustrated in Figure $1-1$ is used as the basis for all the analyses and associated discussions in this report, except where noted otherwise. Further, unless stated otherwise, both the frame and membrane are assumed to be of steel construction. Finally, when we describe the membraneinduced load on the frame we will always give a value corresponding to the total load on the frame whether discussing single- or double-membrane designs. In the case of doublemembrane designs, we will also assume that the initial tension on each membrane is the same (e.g., if we give a total initial membrane tension on the frame, for a doublemembrane design, of $T_{0}=17,500 \mathrm{~N} / \mathrm{m}$ this will imply that each membrane has an initial tension of $8,750 \mathrm{~N} / \mathrm{m}$ ). 


\subsection{RESPONSE TRENDS FOR SINGLE- AND DOUBLE-MEMBRANE MODULES}

\subsection{Some General Load Deformation Response Characteristics}

In this section we describe the performance of double-membrane and single-membrane concepts and point out differences and similarities in the predicted response of the two design approaches. Since Murphy and Sallis (1984) discussed the details of the deformation response of single-membrane modules extensively, we do not repeat these details here. Proceeding from the predicted load deformation response for specific cases, we discuss the various deformation mechanisms and the anticipated effect on the design of these reflective modules.

We analyzed the load deformation response using an incremental large deformation, small strain analysis method described in detail in Murphy (1985) and also using the NASTRAN finite element code. The NASTRAN (Schaeffer 1979) analysis is much more general and not constrained by many of Murphy's (1985) assumptions and thus helps to verify the simpler model for specific cases corresponding to a range of parameters.

Consider the single- and dauble-membrane reflector modules described in Figure 2-1. Let the total average tension level on the frame before lateral pressure loading be $T_{0^{*}}$.

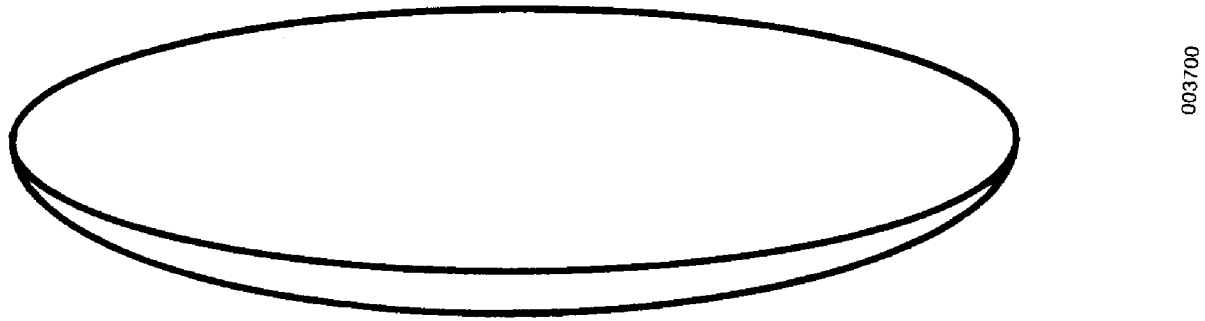

a) $w_{1}$ axisymmetric portion of membrane deformation

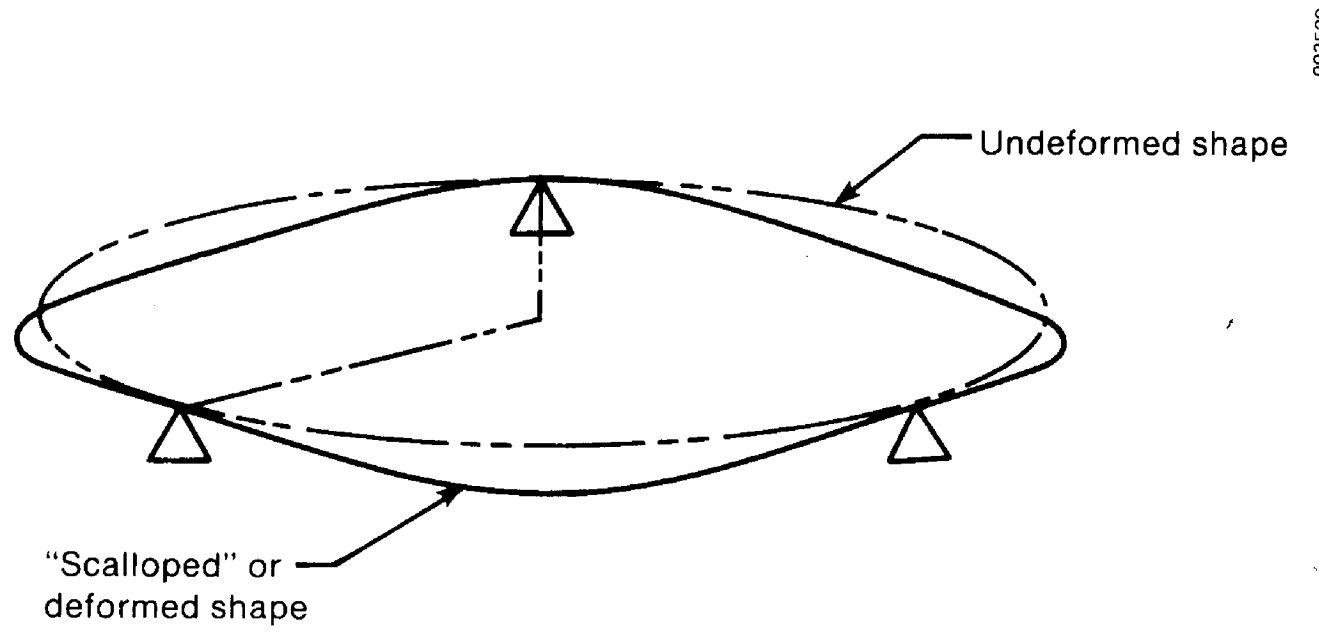

b) $w_{2}$ nonsymmetric, "scalloped," membrane shape caused by support constraints

Figure 2-1. Axisymmetric and Asymmetric Deformation Modes for a Typical Membrane 
For the double-membrane modules, each of the membranes contributes exactly one-half of the radially inward-directed load on the frame. The loading on the membrane and frame because of wind and weight is uniform and perpendicular to the plane of the membrane, and the frame is supported at three points spaced evenly around the circumference. The supports are rigid and offer restraint only in the direction that is perpendicular to the plane. Most of the examples in the main body of this report assume three supports and a nominal 5-m-radius frame.

The deformation response predictions, described in Figures 2-2 through 2-9 and discussed in this report, explain the various performance features of the double-stretchedmembrane module. It is important to distinguish between the axisymmetric and asymmetric deformation contributions (see Figure 2-1) to the total deformation of the membrane reflector surface. The axisymmetric portion arises from a uniform pressure differential across a membrane with a fixed boundary. The asymmetric portion arises from the nonuniform but periodic reaction forces at the supports (see Murphy and Sallis 1984). In all the discussions related to the double-membrane concept, we describe only the optical surface errors resulting from the asymmetric deformation because we assume that a pressure vacuum control has eliminated the error contribution from the axisymmetric membrane deformation. Note, however, that we considered both modes of deformation in the deformation analysis and that it is quite simple to add the axisymmetric deformation portion, if required, to arrive at the total deformation for the membrane.

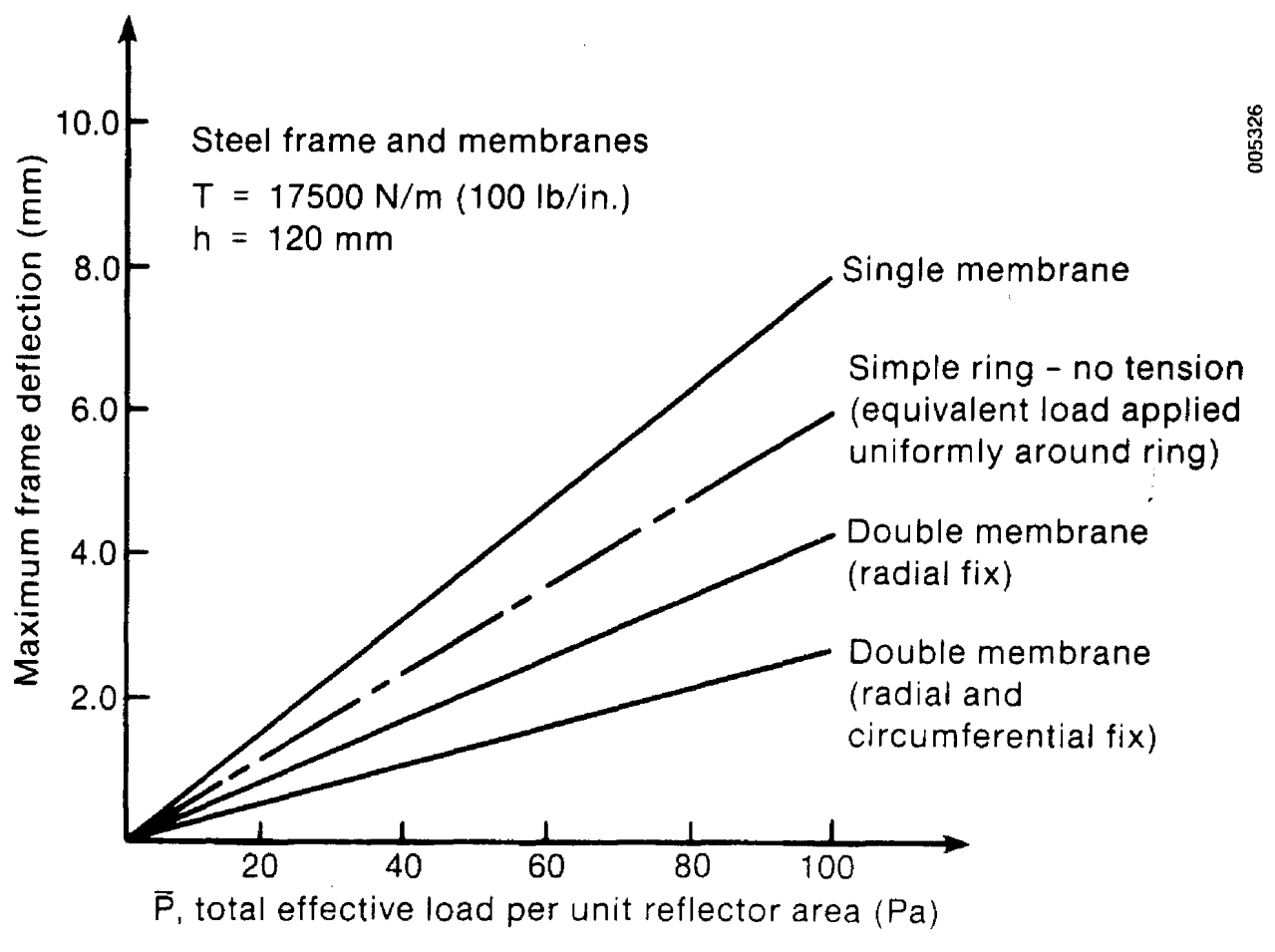

Figure 2-2. Maximum Frame Displacement Midway between the Supports as a Function of the Total Effective Load per Unit Reflector Area 


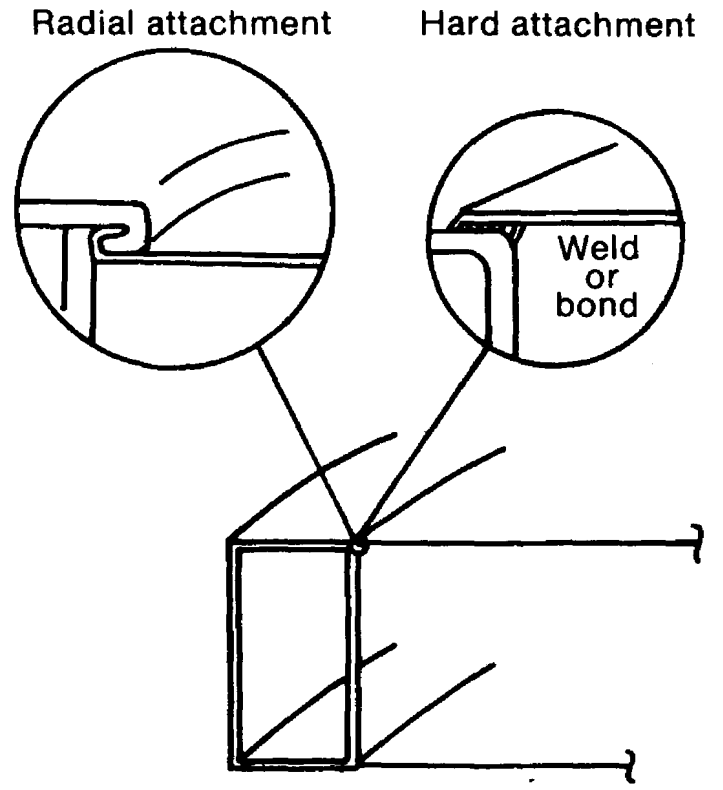

Figure 2-3. Membrane Attachment Details Showing Radial and Hard Attachments
Figure 2-2 shows that the module frame deforms laterally in a linear manner with pressure. Here, the pressure is applied uniformly in a direction perpendicular to the plane of the membrane. Both frame and membranes are made of steel. In Figure 2-2 the maximum lateral deflection of the frame that is measured midway between the three supports is a function of uniform pressure $\bar{P}$ on the membrane for several designs. The curve labeled simple ring corresponds to a uniform line load, which has an integrated total value that is equivalent to a uniform pressure load over the plane of the membrane, and applies to a simple ring without a membrane. Note that in all cases the load on the frame caused by the membranes introduces frame instability response and, for a single-membrane module, the load/deformation response is greater than that of the ring alone. The single-membrane configuration is less stiff to lateral deflections than a simple ring with no membrane tension because of the frame instability response. Double-membrane modules,

however, can overcome the frame instability response and be significantly stiffer than the ring alone, as we can see from the bottom two curves, because of two effects that couple the membrane stiffness with the frame in a manner not possible with the singlemembrane concept. The curve corresponding to the highest overall stiffness and the lowest deflection represents a double-membrane concept where the attachment does not allow the membrane to move independently in either the radial or circumferential direction (i.e., a hard attachment) from the attachment point on the frame (see Figure 2-3). Thus, the membrane not only inhibits the rolling of the frame but the membrane also must strain the same amount as the frame at its attachment point. This bending strain compatibility effect at the attachment is analogous to the membrane acting as an additional flange attached to the frame; we refer to this effect later as the flange effect. The curve above the curve we have been discussing, but below the simple ring curve, represents a double membrane where only a radial fix at the attachment point is used. In this situation, the membrane moves freely in the circumferential direction at the attachment point but not radially (see Figure 2-3). Such a situation may occur if there is a clamp or a periodic attachment at the frame; in this case, only the roll resistance of the frame is enhanced since, as the frame bends, the membrane is free to move in its circumferential direction and is not forced to strain in that direction with the frame.

When the membrane offers bending, roll resistance, or both to the frame at the attachment point, the amount of membrane material that participates in this effect and the level of this effect depends on the stiffness and thickness of the membrane material. This is not the case with single-membrane designs where, in most cases, only the membrane tension influences the frame deflections. The one exception corresponds to situations where the membrane is offset (vertically above the plane passing through the frame center of torsion) and has a hard attachment that adds to the frame bending stiffness as a flange. 
When considering the roll-resistance enhancement that the double membrane with a radial attachment offers to the frame, note that one membrane experiences a local tension increase at a given circumferential attachment position, while the opposite membrane at the same circumferential location experiences a localized tension decrease. The directions of the local tension increments always resist the rotation of the frame associated with nonuniform out-of-plane deformations. Thus, the double membranes add considerable rotational stiffness to the system, and the out-of-plane deformations are correspondingly retarded as well since the out-of-plane bending and frame rotation are strongly coupled. Further, the double membranes interact with the frame under loading normal to the membrane plane even at low tensions. In addition, since the upper and lower membranes have tension increments of equal magnitude but of opposite sign, local radial deformation cannot compensate to make the tension uniform in the upper and lower membranes. Thus, the rotational restraint offered by the membranes leads to in-plane tension increments in the membrane that do not occur in most singlemembrane designs.

For most single-membrane designs where the radial stiffness of the frame is low compared to that of the membrane, the frame adapts radially to keep the membrane tension constant and thus offers little roll restraint. For the offset, hard-attachment, singlemembrane case, however, variations in the membrane tension occur near the frame because of the flange effect; these effects generally dissipate rapidly, moving radially inward on the membrane surface.

Figure 2-4 shows the variation of effective out-of-plane stiffness (defined as the lateral loading per unit area divided by maximum out-of-plane deformation midway between the

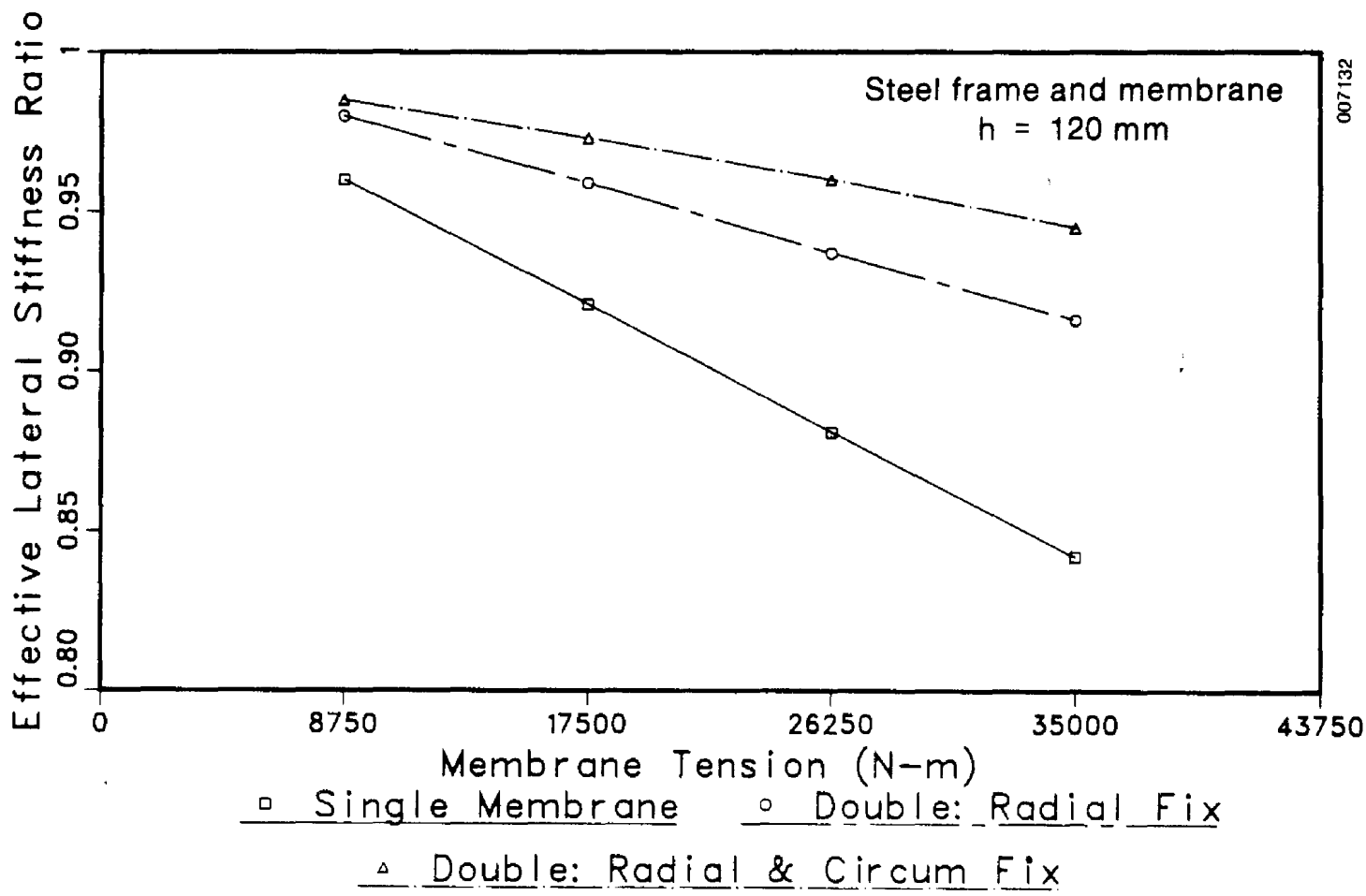

Figure 2-4. Effective Lateral Stiffness Ratio versus Total Membrane Tension on the Frame for Three Module Designs. The frame and membrane are steel; $\mathrm{h}=120 \mathrm{~mm}(4.72 \mathrm{in}$.$) .$ 
supports) caused by lateral loading of the membrane/frame combination as a function of membrane tension. Each curve is also normalized by the stiffness of the corresponding membrane/frame combination at zero initial tension. Several points are noteworthy here: First, since the stiffness changes as a function of tension, the deformation process is nonlinear with changes in tension. Second, all cases experience the decrease in stiffness with the increase in tension. (This decrease in stiffness is caused primarily by the tendency of the ring compressive load in the frame to amplify the deformations caused by the loads that are normal to the plane of the frame, as is discussed more fully in the section describing buckling phenomena.) Third, the decrease in stiffness is less pronounced for a double-membrane concept than it is for a single-membrane concept. Finally, the more effectively the membrane is attached to the frame, the less impact tension increases have on stiffness.

\subsection{Frame Bending Stiffness Effects}

Next, we consider the effect of varying the frame stiffness properties, in particular the cross-section height, assuming a steel frame of constant width and wall thickness. Figure 2-5 shows the flexural and torsional rigidity for the assumed frame (inset), as a function of height. The assumed external loading that induces the deformations illustrated in Figures 2-7 through $2-10$ is composed of two parts, as fllustrated in Figure 2-6. First, we assume that a normal pressure of $10 \mathrm{~Pa}\left(0.21 \mathrm{lb} / \mathrm{ft}^{2}\right)$ acts on the membrane. This corresponds to a mean dynamic wind-loading pressure of about $4.0 \mathrm{~m} / \mathrm{s}$ $(9.0 \mathrm{mph})$ if the heliostat were facing directly into a uniform velocity wind. For the orientation of the collector, this pressure (facing upward with normal being $30^{\circ}$ from vertical) corresponds more closely to the effect experienced by the collector from a $8.0-\mathrm{m} / \mathrm{s}(18-\mathrm{mph})$ uniform wind stream that is parallel to the ground. The second load component is the weight of the structure, and this component is the largest lateral loading component element for the cases considered here. To put this in perspective, at a frame half-height of $150 \mathrm{~mm}$ the normalized weight loading of the frame and membranes, acting normal to the membrane (for the double-membrane concept), is approximately $94 \mathrm{~Pa}\left(2.0 \mathrm{lb} / \mathrm{ft}^{2}\right)$. We have not considered the in-plane component of the weight vector in either the NASTRAN or the simplified analysis because of the relatively high in-plane stiffness when compared to the out-of-plane stiffness for most module designs. Both the frame and the membranes are assumed to be steel, and the membranes are assumed to be $0.254 \mathrm{~mm}(0.010 \mathrm{in}$.) thick; a total tension of $17,500 \mathrm{~N} / \mathrm{m}(100 \mathrm{lb} / \mathrm{in}$.) on the frame is also assumed. Figures 2-7 through 2-9 show the corresponding effect of increasing the height of the frame cross section on the maximum out-of-plane deflection* and both the peak and rms surface slope errors corresponding to the asymmetric component of surface deformation for the assumed loading. The highest curve (largest deflection) in each of these figures corresponds to a single-membrane design that is mounted in the center of the frame as seen in Figure 1-1; the second highest curve corresponds to a double-membrane design with a radial attachment; and the lowest (best performance) curve corresponds to the double-membrane design with a hard attachment. The effect of increasing the height of the frame is quite dramatic.

The relative benefit of double-membrane designs increases with frame height because the membrane offers more effective roll restraint as the membranes spread farther apart and the effective moment arms increase in length. Further, the benefit of hard versus radial attachment decreases as frame height increases because the bending resistance of

* The peak displacement occurs midway between the supports. 


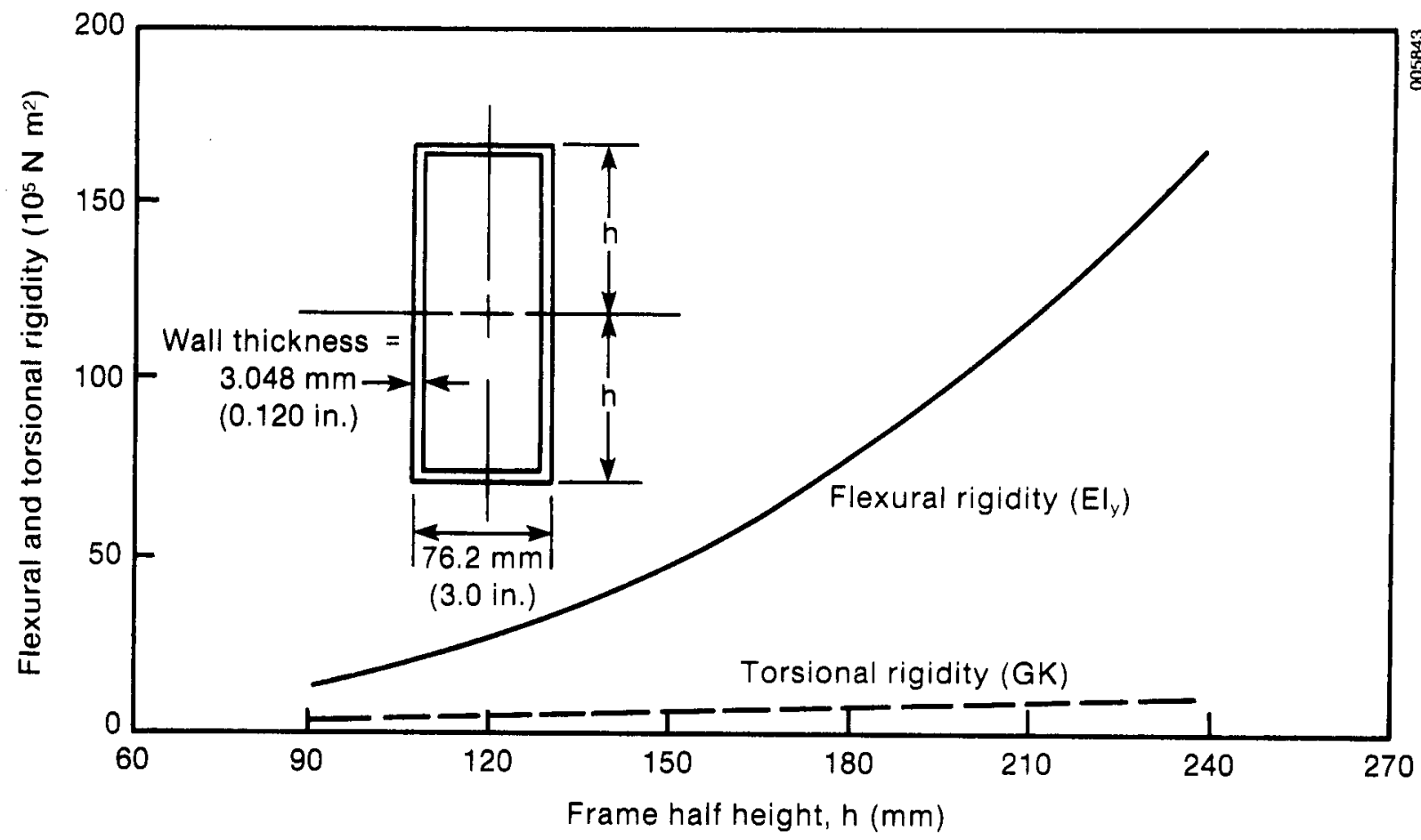

Figure 2-5. Flexural and Torsional Rigidity of Frame as a Function of Frame HalfHeight (for the Cross Section Shown in Inset)

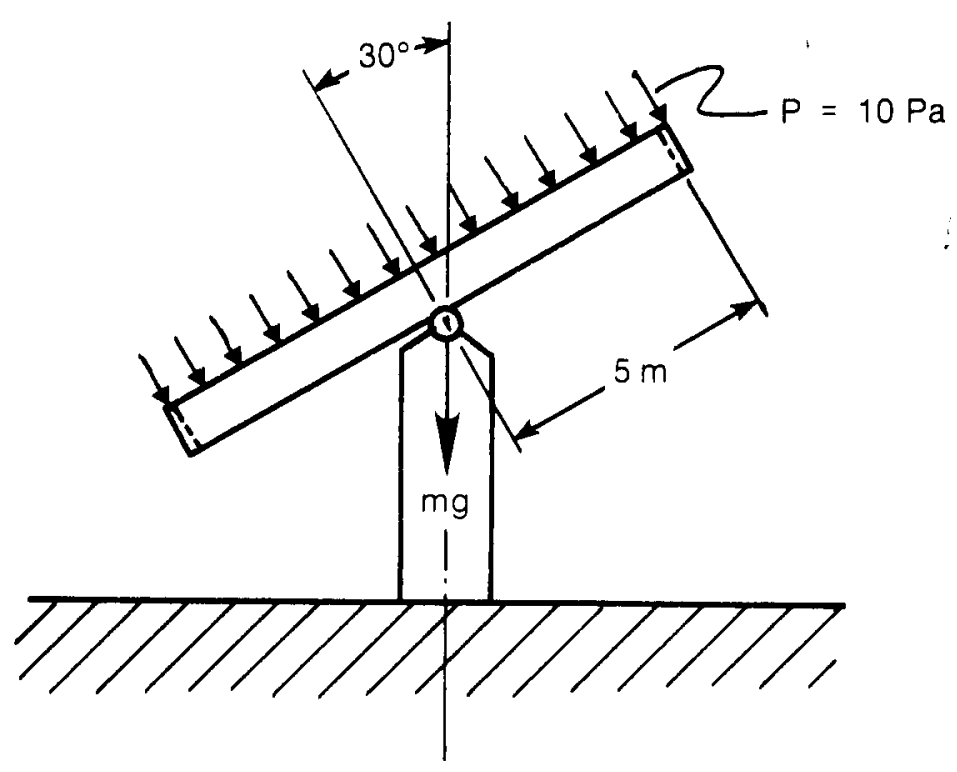

$\stackrel{\infty}{\stackrel{\varpi}{\tilde{g}}}$

Figure 2-6. Gravity and Wind-Induced Loading Assumed for the Modules Considered in Figures 2-7 through 2-10 


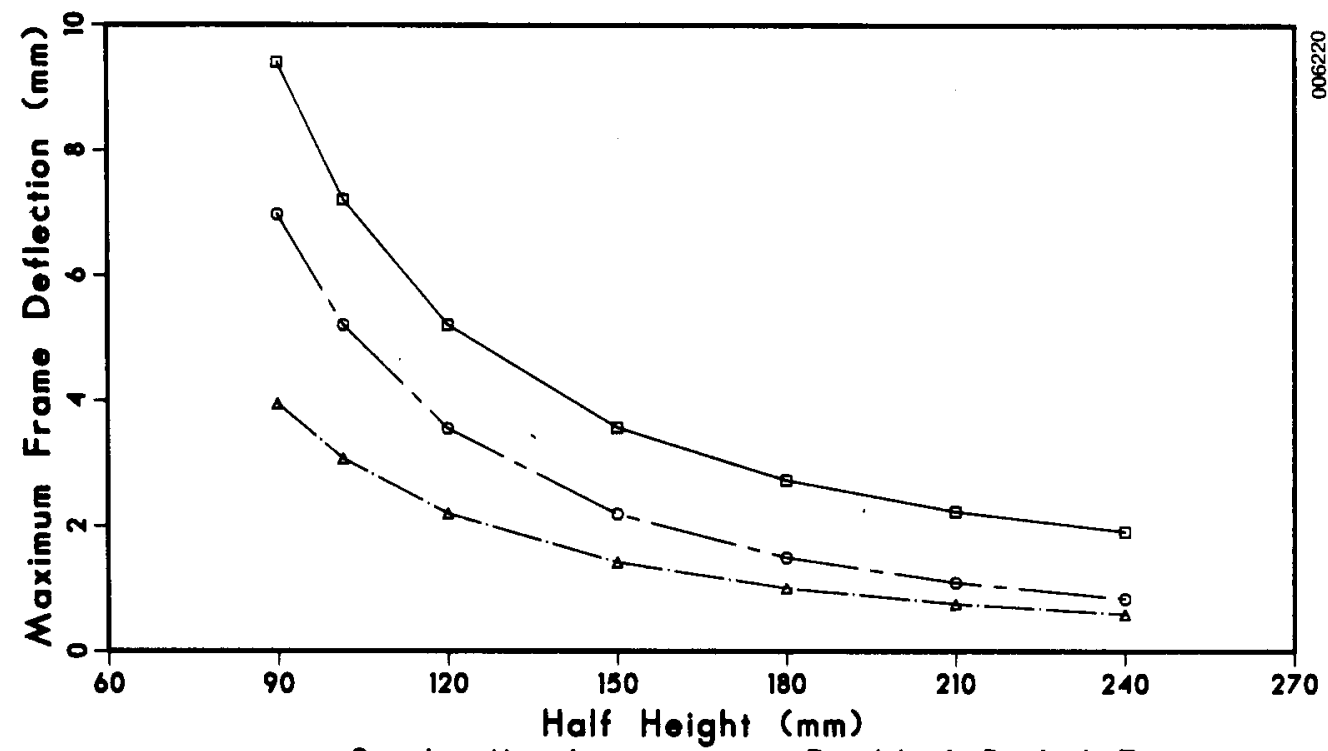

- Single Membrane Double/ Radial Fix

$\triangle$ Double/ Radial \& Circum. Fix

Figure 2-7. Peak Lateral Frame Deformation (Midway between the Supports) as a Function of Frame Half-Height for Single-Membrane, Double-Membrane (with Radial Attachment), and Double-Membrane (with Hard Attachments) Module Designs ( $T_{0}=17,500 \mathrm{~N} / \mathrm{m}[100 \mathrm{lb} /$ in. $]$ )

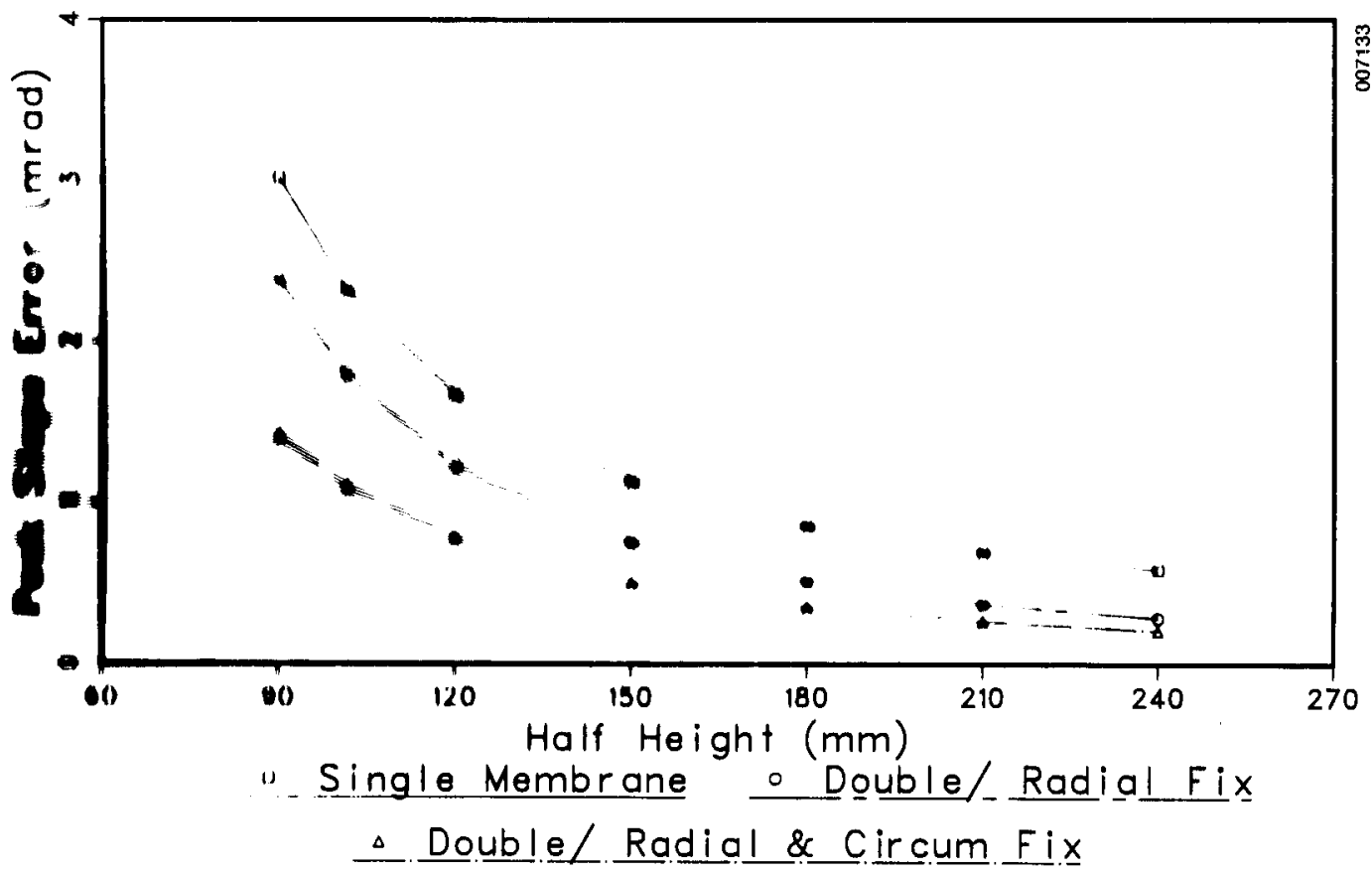

Figure 2-8. Peak Membrane Slope Error Corresponding to the Asymmetric Deformations as a Function of Frame Half-Height for Three Module Designs Considered in Figure 2-7 


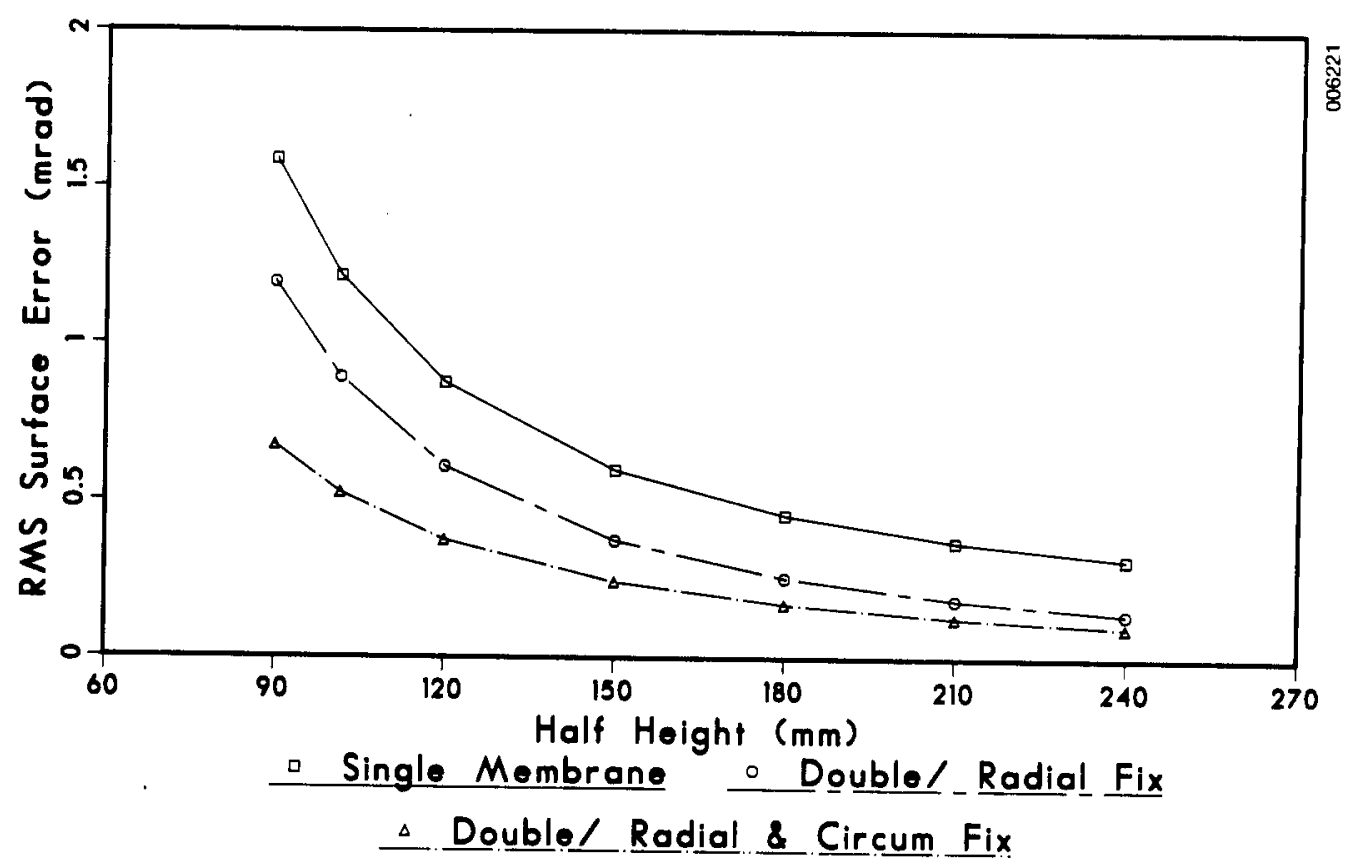

Figure 2-9. Membrane rms Surface Error (Asymmetric Portion) as a Function of Frame Half-Height for the Three Module Designs Considered in Figures 2-7 and 2-8

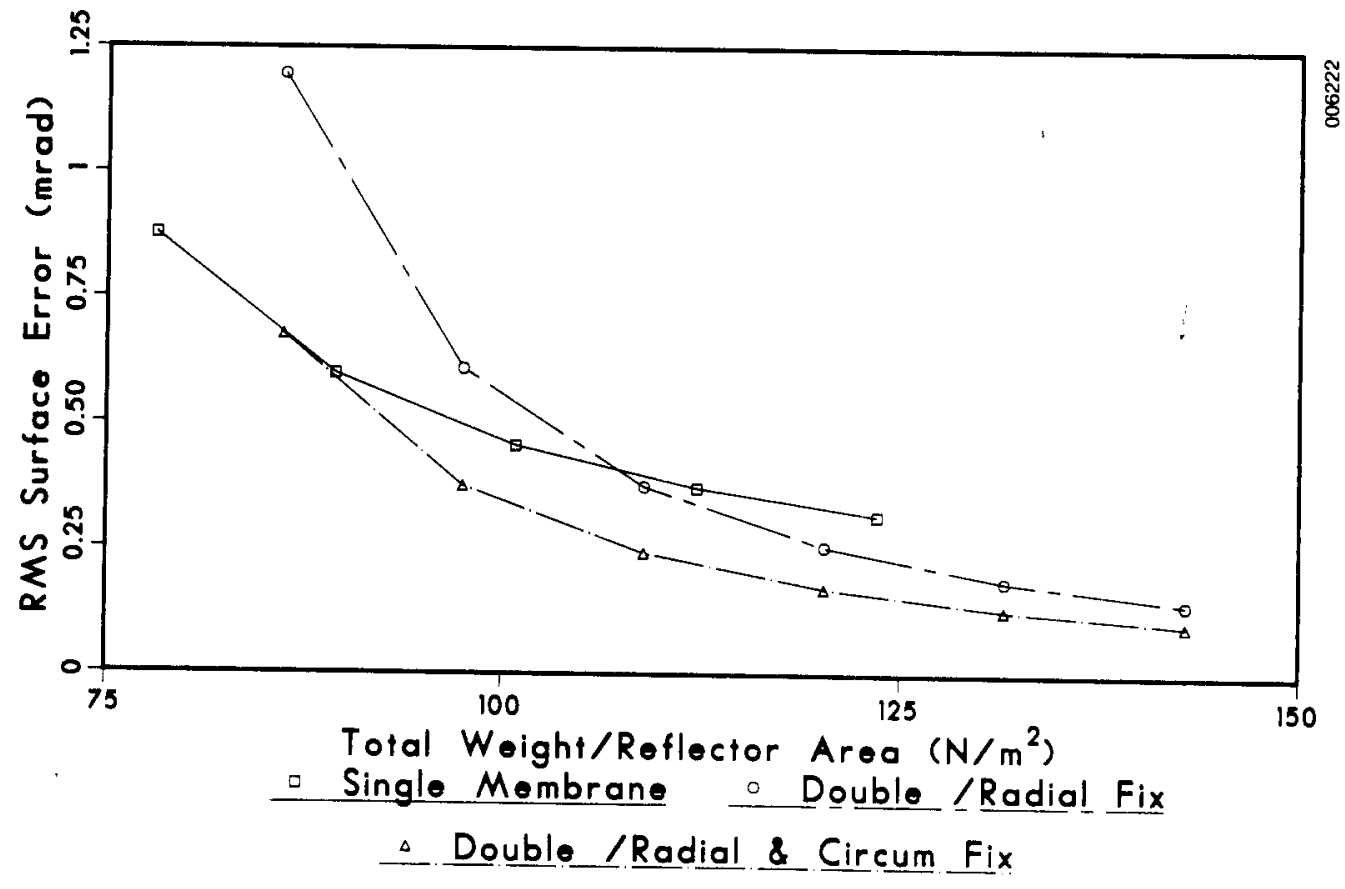

Figure 2-10. Membrane rms Surface Error (Asymmetric Portion) as a Function of Weight per Unit Reflector Area for the Three Module Designs Considered in Figures 2-7 through 2-9 
the frame increases relative to the membrane-induced flange effect discussed previously. Thus, for the cases considered here, the roll-resistance effect is more dominant than the flange-induced effect.

Comparing Figure 2-9 with Figure 2-7 shows that, for all practical purposes, the rms surface error is almost linearly proportional to the peak deformations calculated in Figure 2-7.

Figure 2-10, which illustrates another aspect of the design and response analysis presented in Figures 2-7 through 2-9, shows the rms surface error of the module as a function of the approximate total areal weight of the reflector module. The weight of the module is normalized by the total reflective area. Figure 2-10 illustrates the weight benefit of the different design approaches and shows the potential to design an all-steel module that has a total load module of rms slope error of less than 0.5 mrad and that has an areal weight of less than $100 \mathrm{~Pa}\left(2.1 \mathrm{lb} / \mathrm{ft}^{2}\right)$. To understand the trend of the curves, consider that when larger errors are allowed (i.e., thus requiring lower weights) the fixed-thickness double membranes are the dominant weight element and the singlemembrane design is more weight efficient. However, as more stringent error constraints are imposed on the design, the frame becomes a more dominant weight factor. At an areal weight of about $85 \mathrm{~Pa}$, for the cases considered, the required frame weight increase for the single-membrane design exceeds the weight of the second membrane plus the corresponding frame weight for the double-membrane design (with hard attachments). Thus, the double-membrane design becomes more weight efficient. Note that the wall of the frame and the membranes are fairly thick, indicating that the module has not been optimized and that further weight reductions appear possible.

\subsection{Response Trends Caused by Material Selection}

We also investigated the effect of material selection on the performance of the module, where the loading on the module is the same as in Figure 2-6. Figures 2-11 through 2-13 show the effect of selecting either aluminum, steel, or an aluminum/silicon carbide (SiC) material for a double-membrane module design where hard attachments have been assumed. In each case, both the membranes and frame are assumed to be of the same material and have the frame configuration as shown in Figure 1-1. The total tensioninduced load on the frame is assumed to be $17,500 \mathrm{~N} / \mathrm{m}(100 \mathrm{lb} / \mathrm{in}$.). Table $2-1$ defines the material constants used in the above analysis.

Table 2-1. Material Properties Assumed in Figures 2-11 through 2-13

\begin{tabular}{lccc}
\hline \multicolumn{1}{c}{ Material } & Young's Modulus $(\mathrm{Pa})$ & Density $\left(\mathrm{kg} / \mathrm{m}^{3}\right)$ & Poisson Ratio \\
\hline Steel & $2.07 \times 10^{11}$ & 7800 & 0.3 \\
Aluminum & $7.58 \times 10^{10}$ & 2600 & 0.3 \\
Aluminum SiC & $1.10 \times 10^{11}$ & 2720 & 0.3 \\
\hline
\end{tabular}


Figures $2-11$ and $2-12$, which are analogous to Figures $2-7$ and $2-9$, respectively, show the maximum deflection of the frame between the supports and the rms surface error, each as a function of frame half-height. Figure 2-13, which is analogous to Figure 2-10, shows the rms surface error as a function of area normalized weight. Note that the frame wall and membrane for all cases have the same thickness as the respective steel components and further that none of the modules has been optimized. A potential performance advantage exists when using either aluminum or aluminum/SiC material because the load-induced deformations and surface errors are controlled mainly by the weight of the structure. In such situations, the required mass in the structure is most strongly dependent on the density of the material used and much more weakly on the strength-toweight ratio of the material. Thus, for the cases studied here, the performance is more responsive to changes in weight or mass than to corresponding changes in material stiffness. Figure $2-13$ shows that either aluminum or aluminum/SiC performs similarly from a weight perspective, but each offers a significant weight performance improve- ment relative to the steel design for the loading case investigated. Although first observations indicate that a cost of aluminum (per unit mass) that is three times the cost of steel is justified, we need to consider numerous other issues. Weldability, minimum acceptable wall and membrane thickness, ease of handling and shipping, and impacts on fabrication must be considered in future trade-offs to assess the potential benefits of using different materials.

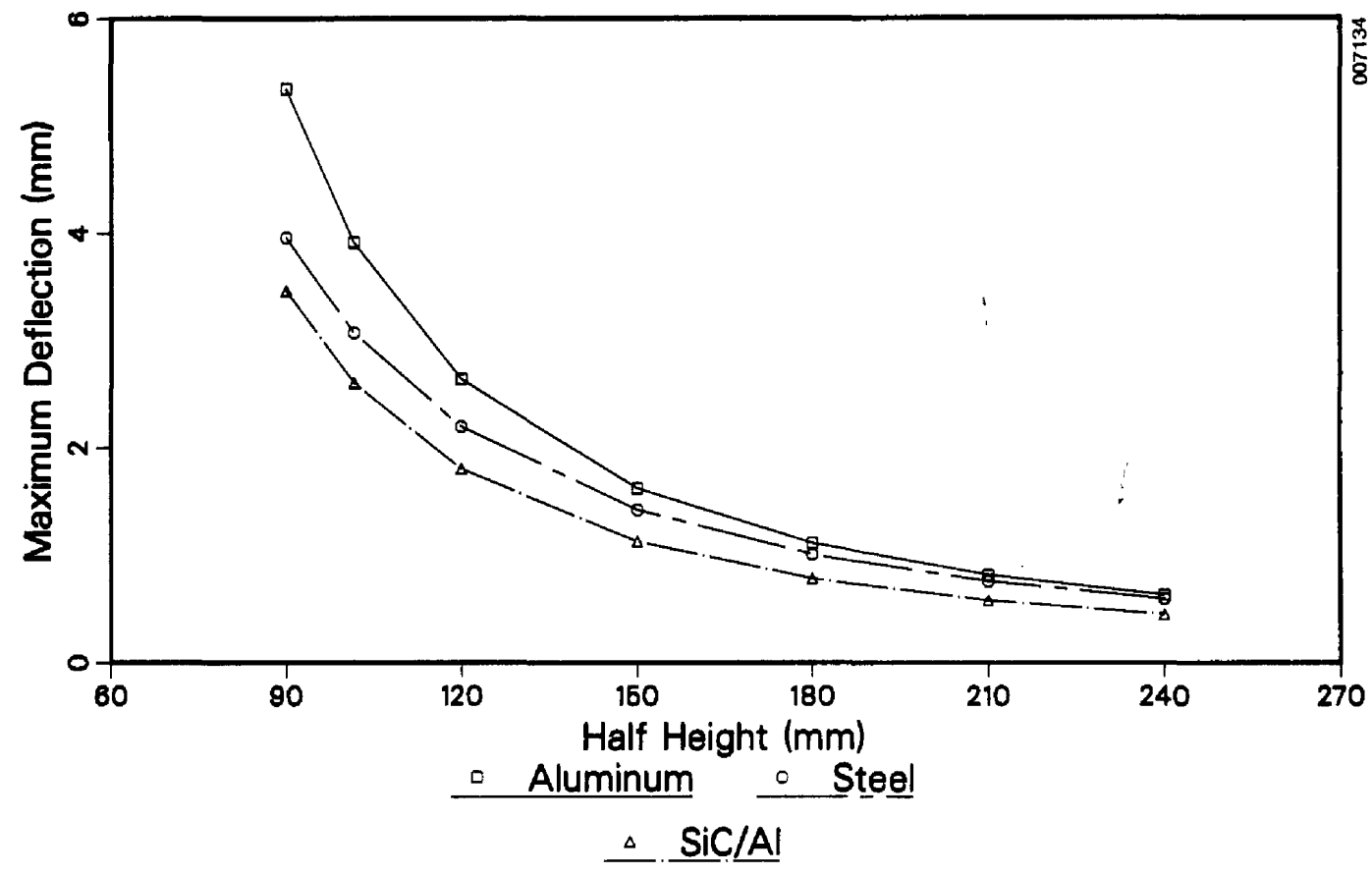

Figure 2-11. Peak Lateral Frame Deformation (Midway between the Supports) as a Function of Frame Half-Height for Si-C/Al, Aluminum, and Steel Double-Membrane Designs (Hard Attachments Are Assumed) $\left(\mathrm{T}_{0}=17,500 \mathrm{~N} / \mathrm{m}[100 \mathrm{lb} / \mathrm{in}].\right)$ 


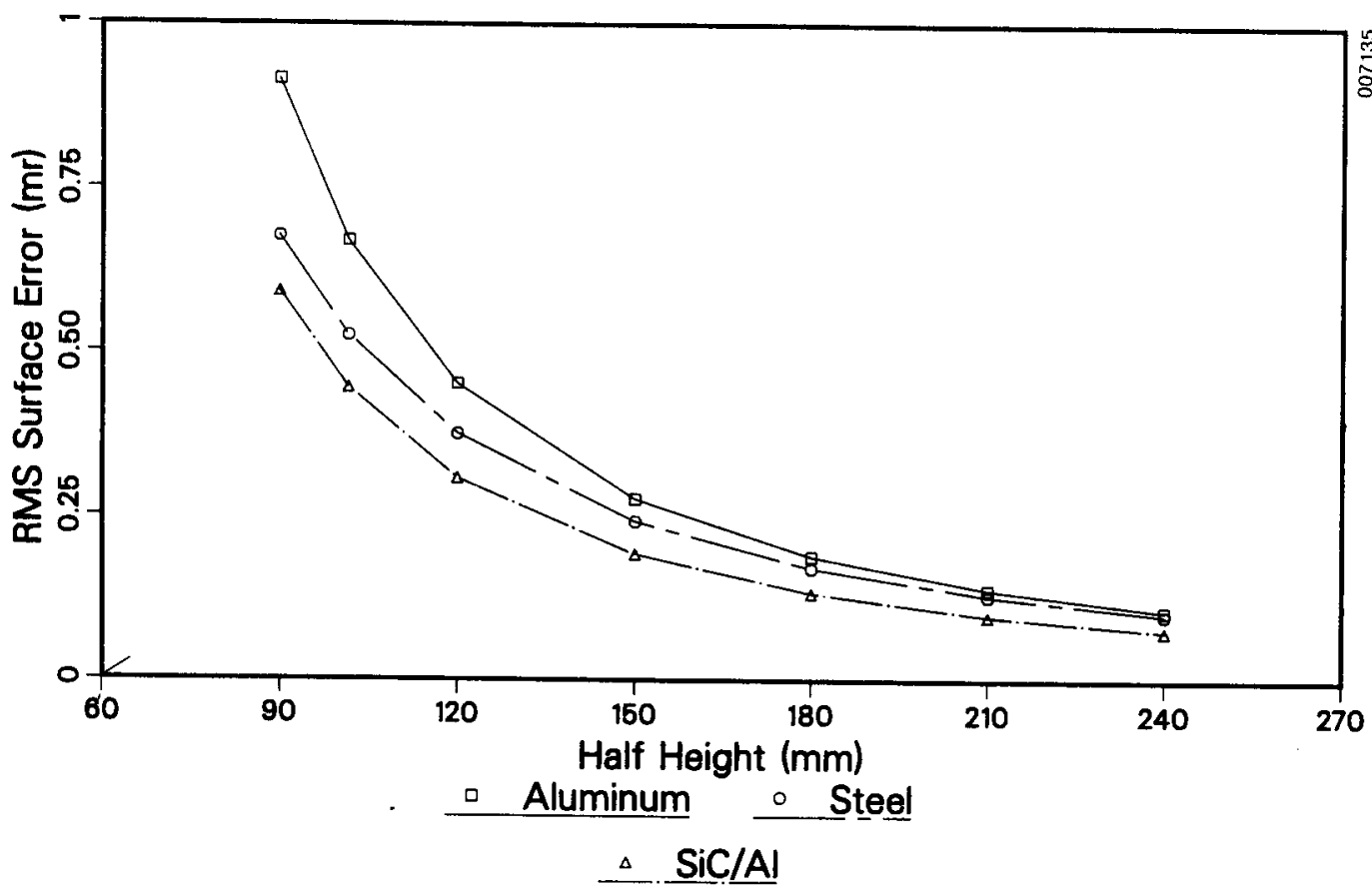

Figure 2-12. Membrane rms Surface Error (Asymmetric Portion) as a Function of Frame Half-Height for SiC/Al, Aluminum, and Steel Double-Membrane Design (Hard Attachments Are Assumed)

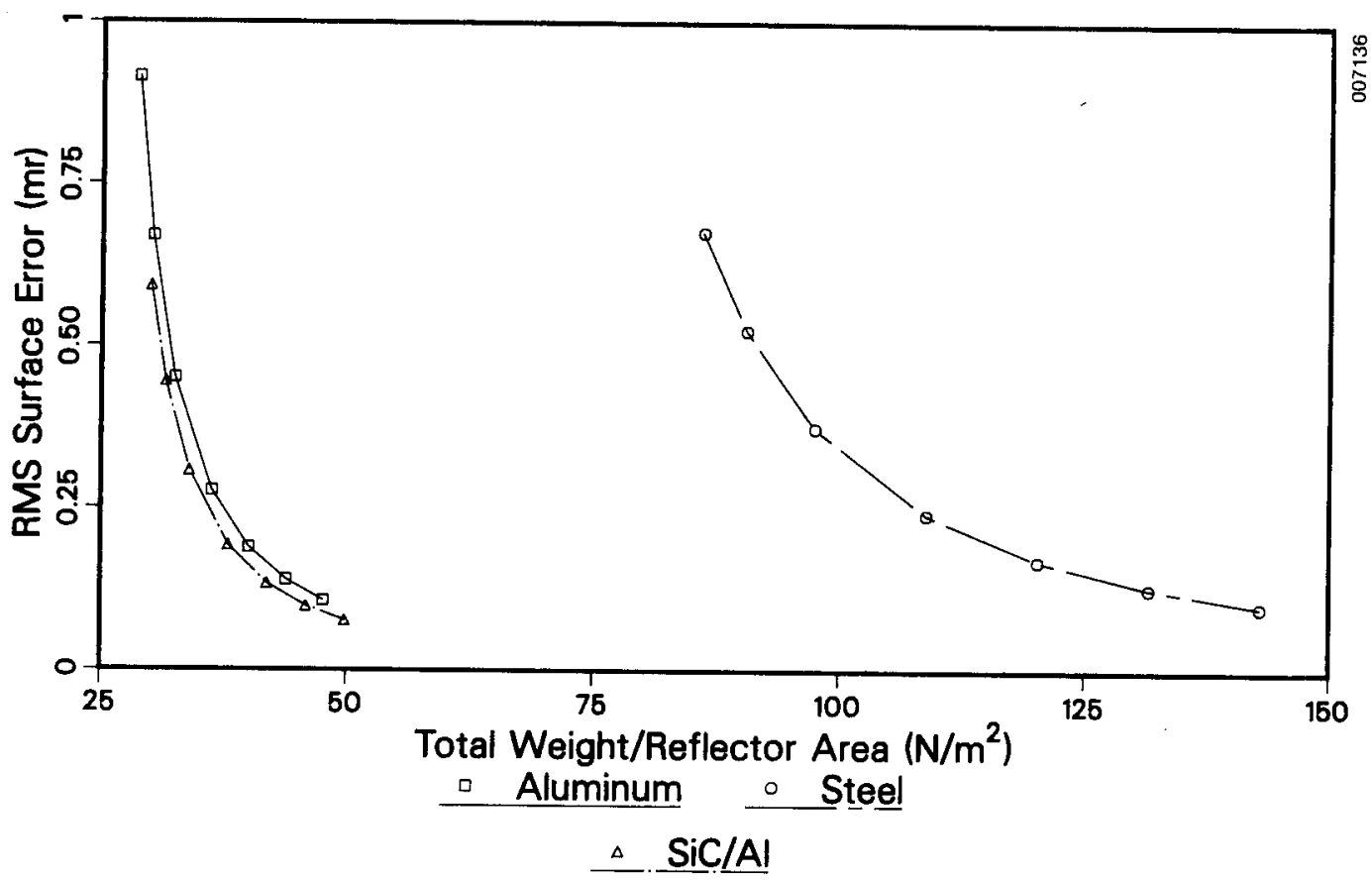

Figure 2-13. Membrane rms Surface Error (Asymmetric Portion) as a Function of Total Weight per Unit Reflector Area for SiC/A1, Aluminum, and Steel Double-Membrane Design (Hard Attachments Are Assumed) 


\subsection{Frame Torsional Stiffness Effects}

Figures 2-14 and 2-15 show the effect of holding either the flexural rigidity constant and varying the torsional rigidity, or conversely holding the torsional rigidity constant and varying the flexural rigidity. Two design cases (i.e., for a single-membrane design and for a double-membrane design with a simple radial attachment) are plotted, assuming a 90-Pa load on the membrane (no weight loading is considered here). $\mathrm{h}$ is assumed to be $101.6 \mathrm{~mm}(4 \mathrm{in})$. In Figure $2-14$ the flexural rigidity is constant (i.e., EI = $\left.1.77 \times 10^{6} \mathrm{~N} \cdot \mathrm{m}^{2}\right)$, and the torsional rigidity varies. For the double-membrane concept (bottom curve), the torsional rigidity has little effect on the maximum out-of-plane deflection of the frame. However, for the single-membrane concept (upper curve), the results indicate a significant effect on the response. In Figure $2-15$, where flexural rigidity varies but torsional rigidity is held constant (i.e., GK $=0.38 \times 10^{6} \mathrm{~N} \cdot \mathrm{m}^{2}$ ), a significant impact occurs on the module deformation response for both the single- and double-membrane response concepts. Thus, from a design perspective, torsional rigidity is not an important feature for the double-membrane concept (at least for the relatively stiff membranes and attachments investigated to date); but for single-membrane concepts or in situations where the membrane attachment and possibly membrane stiffness are quite low, the torsional rigidity is of significant importance.

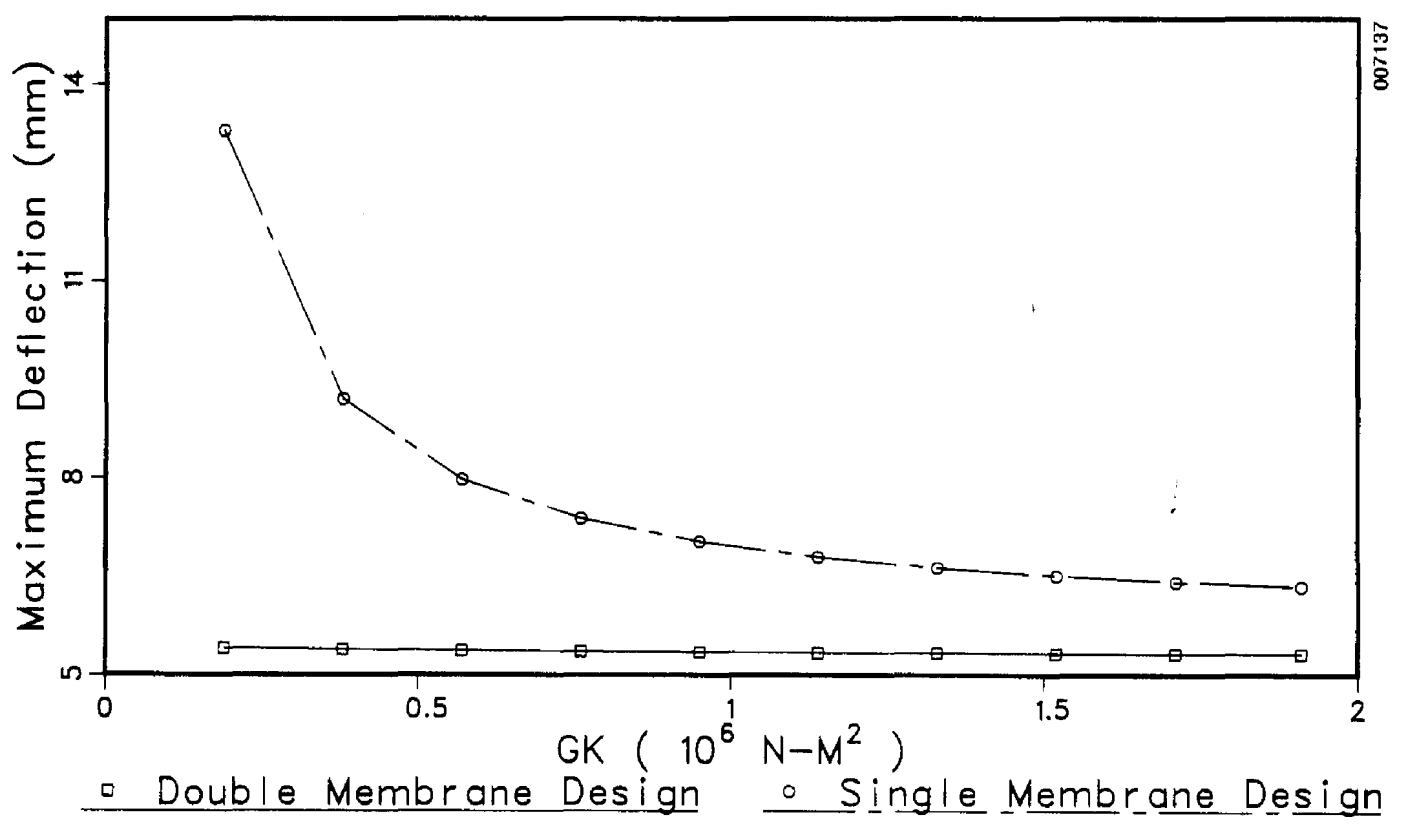

Figure 2-14. Maximum Vertical Displacement of Frame (Midway between Supports) as a Function of Out-of-Plane Frame Bending Rigidity (EI) for Single- and Double-Membrane (with Radial Attachments) Designs at a Constant Value of Torsional Rigidity $\left(\mathrm{GK}=0.38 \times 10^{6} \mathrm{~N} \cdot \mathrm{m}^{2}\right)$; pressure load only $(\mathrm{P}=90 \mathrm{~Pa})$. 


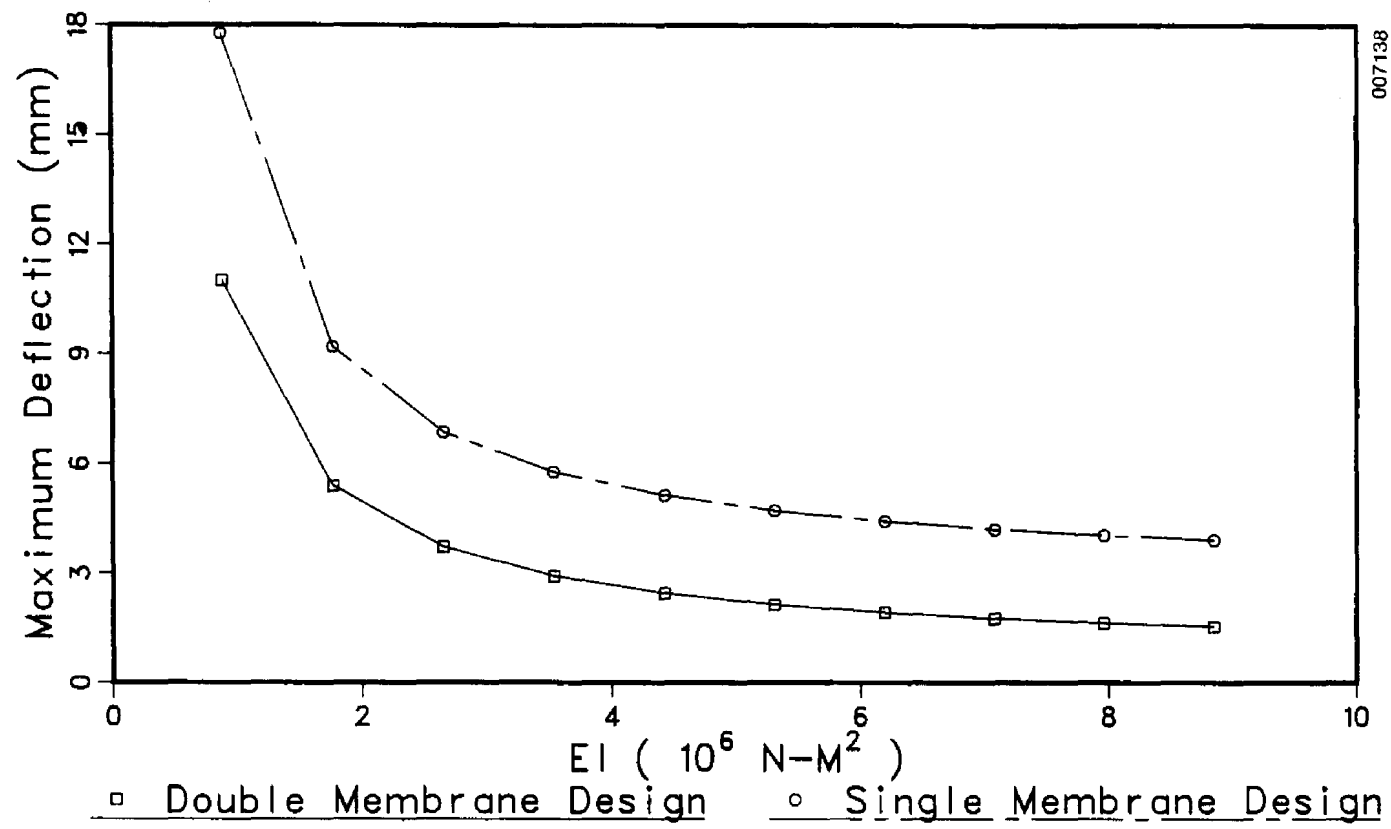

Figure 2-15. Maximum Vertical Displacement of Frame (Midway between Supports) as a Function of Frame Torsional Rigidity (GK), for Single- and DoubleMembrane (with Radial Attachments) Designs at a Constant Value of Frame Bending Rigidity $\left(E I=1.77 \times 10^{6} \mathrm{~N} \cdot \mathrm{m}^{2}\right)$; pressure load only $(\mathrm{P}=90 \mathrm{~Pa})$.

\subsection{Membrane Stiffness Effects}

Figure 2-16 illustrates the effect of decreased membrane stiffness on the lateral response of the frame/membrane combination to pressure loading only (i.e., no weight effects). Note that the frame is still steel and the total membrane load on the frame is $17,500 \mathrm{~N} / \mathrm{m}(100 \mathrm{lb} / \mathrm{in}$.). Here the maximum frame deflection between the supports is a function of frame half-height for one single-and two double-membrane designs. The two double-membrane designs have lowered moduli as indicated in the figure. One could also infer that the trend shown in Figure 2-16 for decreased membrane stiffness is similar to the trend expected for a decrease in attachment stiffness. Even with reduced moduli the double-membrane concept provides benefits but, as the membrane stiffness becomes very low, the double-membrane response approaches the response of a single-membrane concept.

Figure 2-17 illustrates the effects of membrane modulus on the effective lateral stiffness of the module, where a steel frame (as shown in Figure 2-5) with a half-height of $150 \mathrm{~mm}$ is used. The tension is assumed to be constant at $17,500 \mathrm{~N} / \mathrm{m}(100 \mathrm{lb} / \mathrm{in}$. $)$. Membrane thickness is constant at $0.254 \mathrm{~mm}(0.010$ in.) as considered before. Figure 2-17 considers both a single-membrane design and a double-membrane design with a hard attachment, and only the modulus of the membrane is allowed to vary. The membrane material modulus greatly affects the performance of the double-membrane design. Thus, higher moduli membranes result in better performing double-membrane designs. 


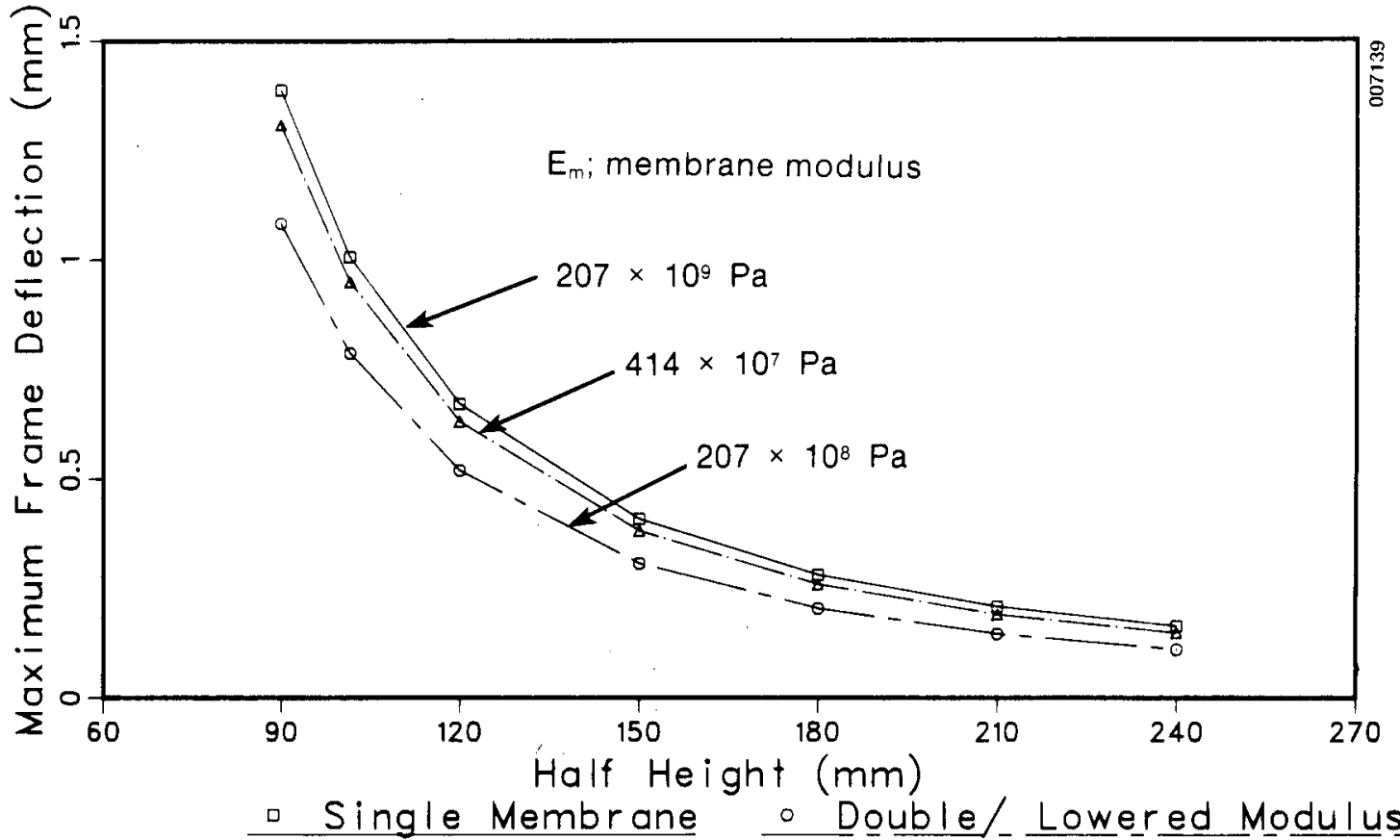

\section{$\triangle$ Doublel Polymer Membrane}

Figure 2-16. Stretched-Membrane Performance as a Function of Frame Half-Height for a Single-Membrane and Two Double-Membrane Designs with Lowered Membrane Moduli; $\mathrm{P}=10.0 \mathrm{~Pa}$; no weight effects.

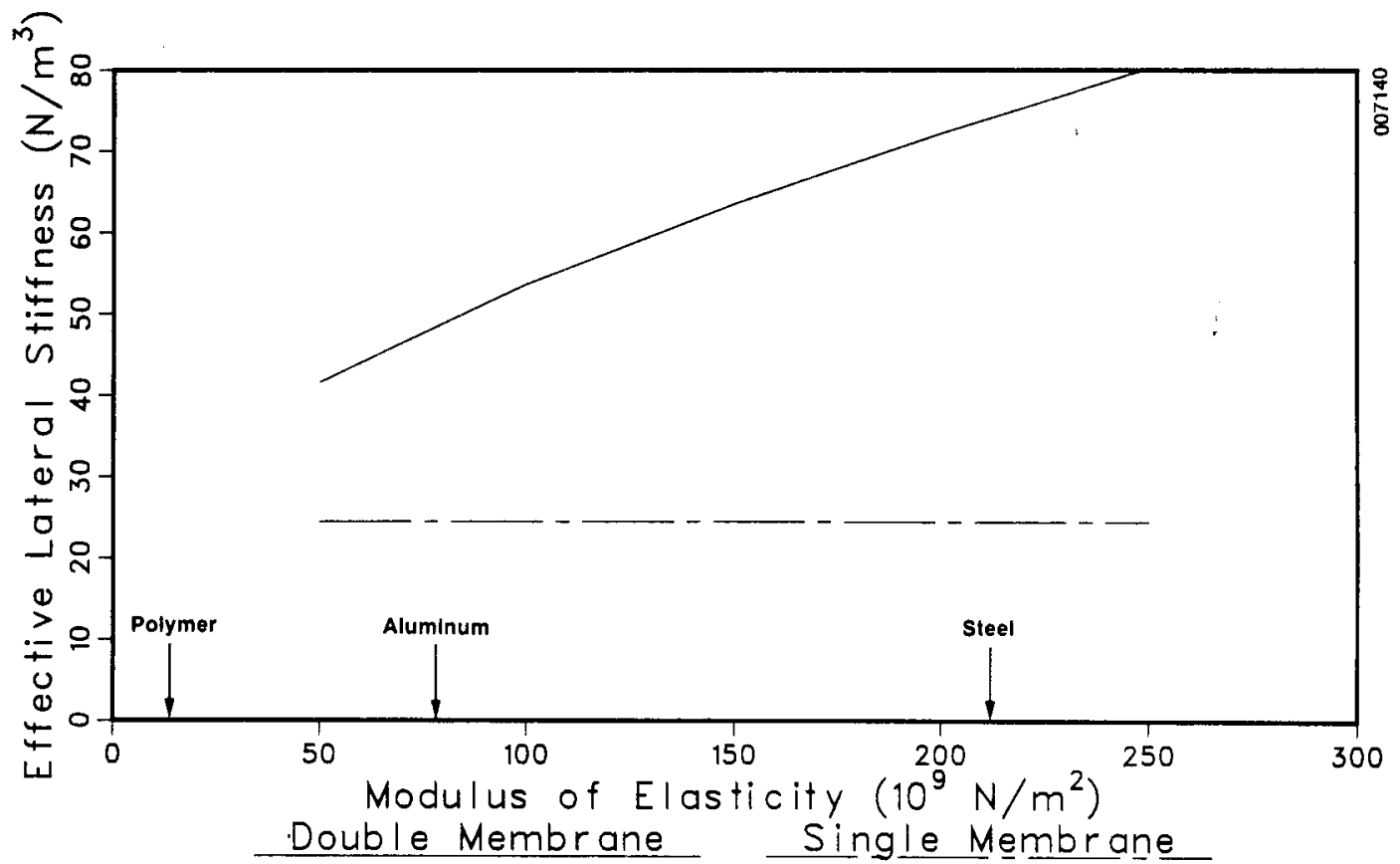

Figure 2-17. Effective Lateral Module Stiffness as a Function of Membrane Modulus for Single- and Double-Membrane (with Hard Attachments) Designs; $\mathrm{h}=150 \mathrm{~mm}$. 
Further, the double-membrane concept approaches the performance level of the singlemembrane design (from a stiffness perspective) as the moduli of the membrane approach zero. For reference, the figure also shows the moduli corresponding to steel, aluminum, and a fairly weak polymer material.

\subsection{Effects Caused by Changes in Frame Radius and Number of Supports}

Up to this point we have considered a nominal constant radius of $5.0 \mathrm{~m}$ and three supports for the membrane module concepts investigated. In the next three figures we look at the effect of changing the radius and the number of supports. For Figures 2-18 through 2-20, we assume that $T_{0}=17,500 \mathrm{~N} / \mathrm{m}$, steel design is as in Figure 1-1, construction is double membrane with hard attachments, and loading is as shown in Figure 2-6. Changing the number of supports can have a dramatic effect as seen in Figure 2-18, where the maximum frame deflection is a function of the number of simple (and infinitely rigid) frame supports for a fixed-frame geometry $(R=5 \mathrm{~m}$ and $\mathrm{h}=101.6 \mathrm{~mm})$ and membrane tension state. Increasing the number of supports dramatically reduces the deformations between the supports. The impact of the performance improvement on the rms surface error will be significant but not quite as pronounced as one might imply from Figure 2-18. This is because the rms surface error is roughly proportional to the peak deformation amplitude times the square root of the number of supports (Murphy and Sallis 1984; Murphy 1985). This effect is shown in Figure 2-19, which plots the weightper-unit area of the reflector module as a function of the number of supports for several fixed values of rms surface errors. The loading and frame design are the same as those discussed in Figure 2-18, but the frame height is adjusted to result in the specified rms surface error. Note also in Figure 2-19 that we consider only the membrane/frame combination and not the weight of the supports. Figures 2-18 through 2-20 indicate

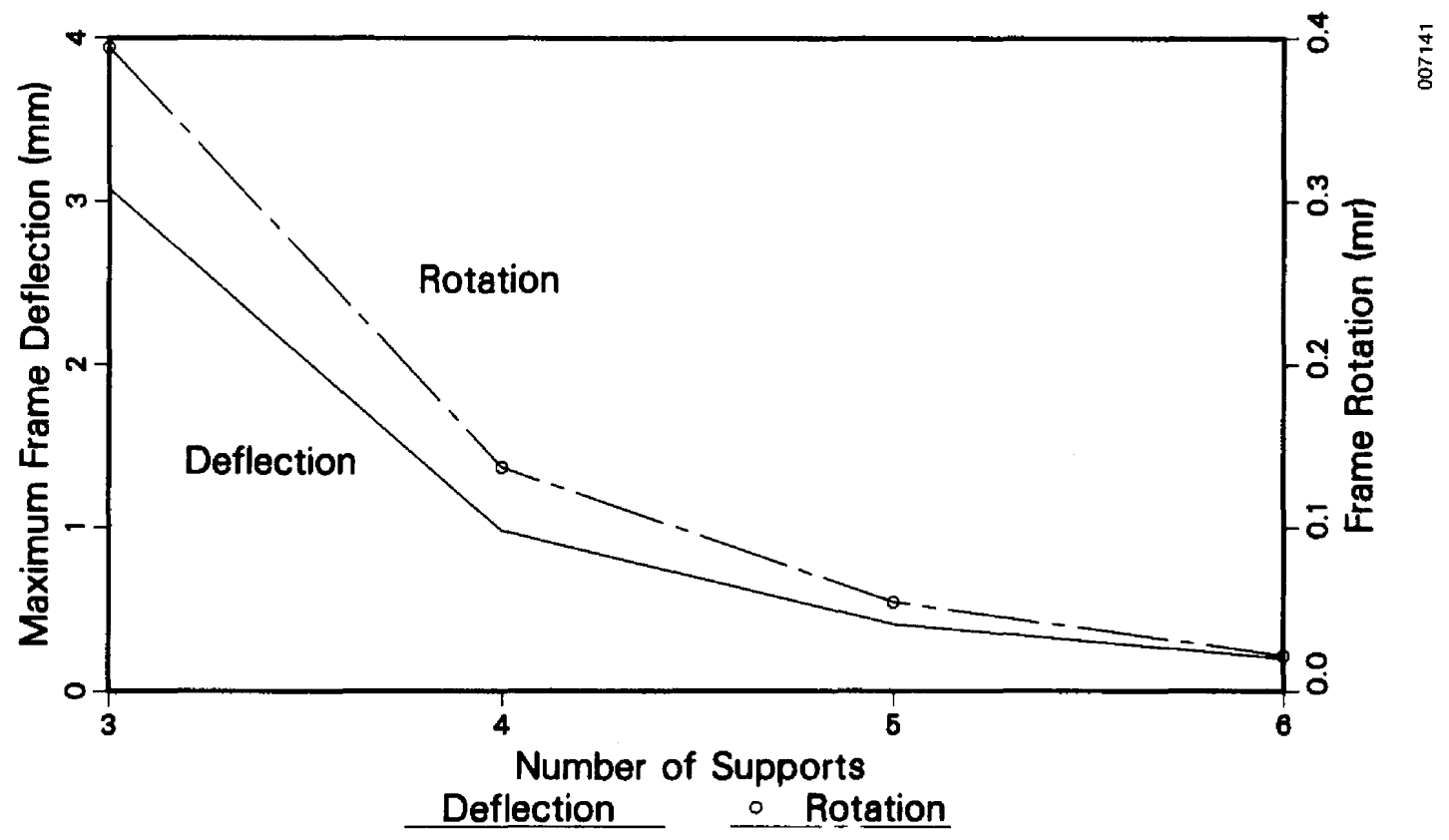

Figure 2-18. Maximum Frame Deflection and Rotation versus the Number of Supports; $\mathrm{R}=5 \mathrm{~m}, \mathrm{~h}=101.6 \mathrm{~mm}, \mathrm{~T}_{\mathrm{O}}=17,500 \mathrm{~N} / \mathrm{m}$; double-membrane design with hard attachments. 


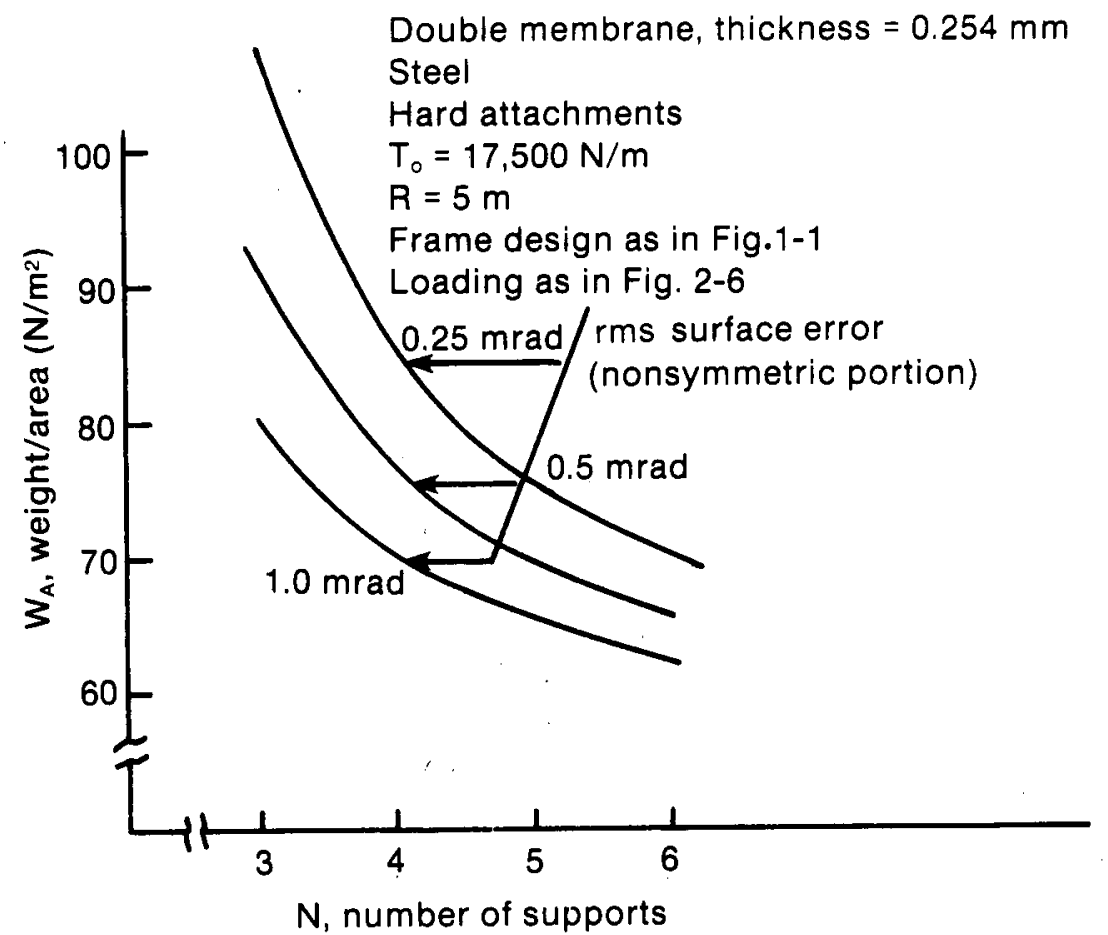

Figure 2-19. Areal Weight versus the Number of Supports for Several Prescribed rms Surface Error Levels; $R=5 \mathrm{~m}, \mathrm{~T}_{0}=17,500 \mathrm{~N} / \mathrm{m}$; double-membrane design with hard attachments.

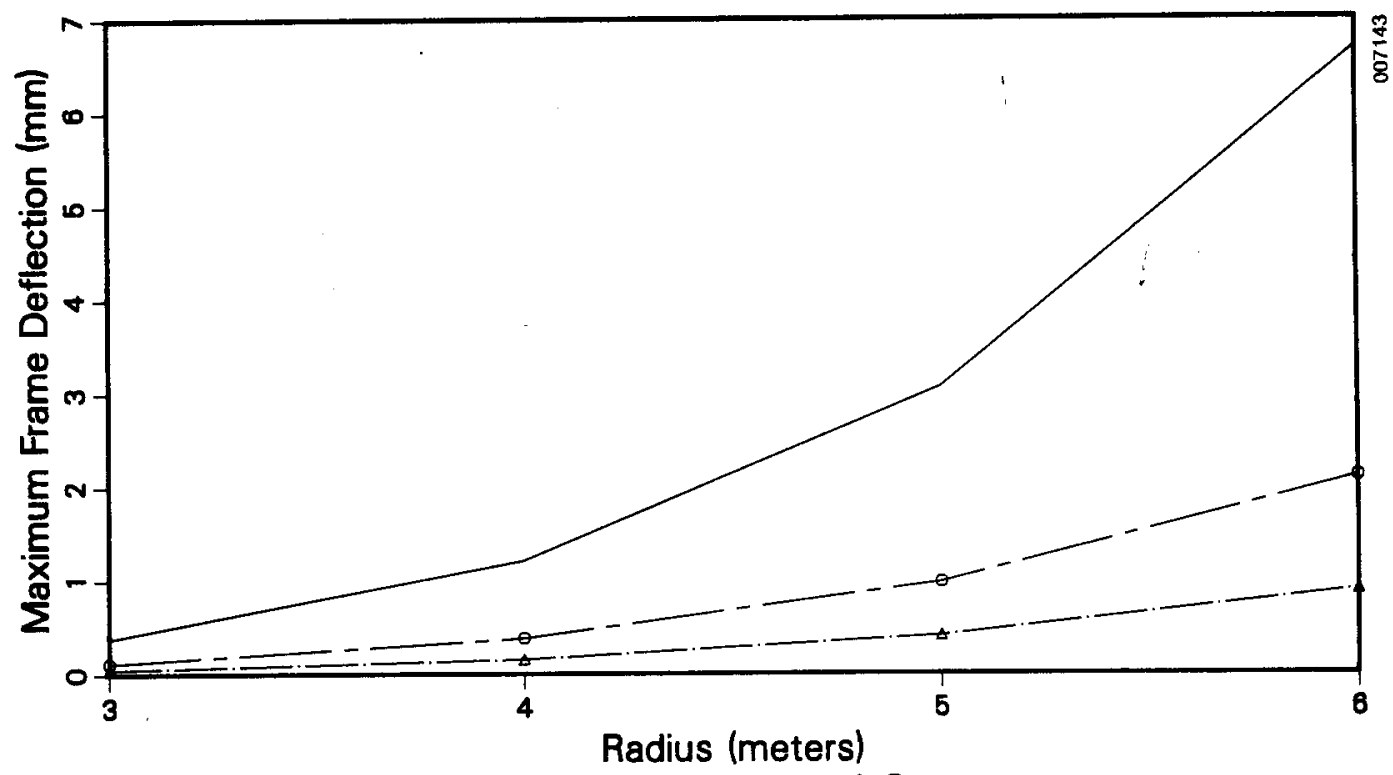

3 Supports 04 Supports

\section{$\triangle 5$ Supports}

Figure 2-20. Maximum Frame Deflection as a Function of Frame Radius for Designs Using Either Three, Four, or Five Evenly Spaced Simple Rigid Supports; $h=101.6 \mathrm{~mm}, \mathrm{~T}_{0}=17,500 \mathrm{~N} / \mathrm{m}$. 
potential advantages to using more than three supports to limit load-induced deformations and the corresponding reductions in optical quality. In addition, one could possibly use more supports to limit or correct for low-frequency, out-of-plane initial frame deformations. These apparent advantages, however, must be weighed against the possible deleterious effects of real supports with finite flexibilities and the additional material requirements, complexity, and costs of more supports.

Finally, Figure 2-20 indicates the impacts associated with radius variations, but with a constant frame cross section $(h=101.6 \mathrm{~mm})$. For the configuration (see inset Figure 2-20) considered, Figure 2-20 shows the maximum frame deflection or a function of frame radius for several different support configurations. Here one sees the strong effect of variations in radius and support number; however, the deflection sensitivity to increased radius decreases with increased number of supports.

\subsection{Some Thoughts on In-Plane Deformations}

Up to this point, the discussions have not addressed the issues of net-in-plane frame deformation. The issue has, however, been implicitly addressed in all of the NASTRAN analyses and was not found to be a significant issue. Furthermore, analyses that have been done (Murphy and Sallis 1984; Murphy 1985) considered the net-in-plane deformation that results from the membrane pretension.

In the single-membrane designs considered in this report, the in-plane deformation increments resulting from the application of a load to the structure that is perpendicular to the membrane plane do not result in a significant structural response.* Further, in the cases considered in this report, the combination of relatively high initial membrane tension and relatively small axisymmetric deformation resulted in very little change in the average membrane stress state, or the net load on the frame. This is because, for the cases considered, the radial stiffness of the frame is quite small compared to that of the membrane. This response, however, could be a concern if the membrane stiffness is quite low, as with a low-strength polymer membrane, or if the radial stiffness of the frame is quite high. For the symmetric double-membrane design, the in-plane frame deformation is of little concern because essentially no effects are present that would cause a significant net-in-plane deformation of the frame once the pretension is applied. There is, however, rotation of the frame that interacts with the membranes that retard the roll motion of the frame, as discussed earlier. Further, in either single- or doublemembrane designs, the bending flange effect of the membrane does affect the membrane stress near the membrane; however, no net radial deformation of the frame will result from this particular deformation mechanism.

In addition to the relatively low stiffness in the radial direction of the frame compared to the membrane, another reason for these results is that in the absence of a membrane, the in-plane frame deformation is significantly decoupled (in the linear approximation) from the out-of-plane deformations and rotations (Murphy and Sallis 1984; Murphy 1982); this situation is quite different from the strong coupling between rotations and out-ofplane deformations of the frame.

* A simple analysis presented in Appendix B illustrates the impact on the radial membrane/frame interaction when large axisymmetric deformations that are perpendicular to the plane of the membrane are considered. In Appendix $B$, the membrane deformations are considered to be large enough for membrane diaphragm stretching. 


\subsection{A Potential Problem with Double-Membrane Modules}

In all cases studied and discussed in this report, the membranes remained in tension under the applied loads. From a design perspective this is a requirement because the membrane cannot sustain compressive loads. If a positive tension is not maintained everywhere in the membrane, the membrane will buckle in the region of zero tension and unacceptable surface distortions will result. Further, the membrane/frame combination will experience considerably different structural responses in the local region of zero membrane tension. We have found from additional analyses, not discussed in great detail here, that localized unloading of the membrane can occur in some situations. This unloading results primarily from frame rotation, which accompanies the out-of-plane frame displacement between the supports under the applied loads. We have observed local unloading of membranes in areas where very low initial tensions and three supports were assumed. This issue should be investigated during the design process and is easily accomplished when using a computer code such as NASTRAN. Also, one can use the simple analytical model described in Murphy (1985) to estimate when a load reversal will occur for a specific module design and loading. 


\subsection{STABILITY CONSIDERATIONS}

\subsection{General Considerations}

The stretched-membrane module is an initially stressed structure with potentially large net compressive loads in the frame. Because of these compressive loads, stability issues become a concern. First, stability is a major design issue to appropriately size the frame and other support elements and to optimize the system configuration to reduce the cost and size of the structural elements required. Also, we will show later that the proximity to the critical tension level determines the level at which initial imperfections and deformations caused by lateral loading are amplified. This issue was partially addressed in Section 2.1 where we showed how initial membrane tension affects the stiffness of the membrane/frame combination subject to loading normal to the plane of the membrane (see Figure 2-4). Moreover, the proximity to the critical tension level affects the degree to which large nonlinear deflections start to dominate the deformation process. Further, an understanding of the load-deformation response, as tension-induced instabilities are approached, leads to a more complete understanding of the overall response process and the potential collapse mechanisms.

Extensive work has been done to determine the critical buckling loads for structures (e.g., the classical work by Timoshenko [1961] and more recent developments discussed by Thompson and Hunt [1973 and 1983]). We will not attempt to summarize the large data base available in the literature. We mention only that both purely analytical and combined analytical and experimental procedures have been developed and are applicable to studying the response of stretched-membrane modules. We will discuss the approaches used in this analysis more fully later in this report.

We use a number of procedures to study the buckling process of the single-membrane module. First, we employ the NASTRAN computer code (Schaeffer 1979) to determine the buckling and postbuckling process for a specific ring frame, and also for a frame/membrane combination. Also, we develop and demonstrate the validity of a simple eigenvalue solution for estimating the buckling load of the frame/membrane combination. Finally, as a check we employ the Southwell (Roorda 1965 and 1967) procedure using the previously calculated prebuckling deformations (from NASTRAN) to also estimate the buckling load.

The inverse Southwell procedure was quite helpful in studying the buckling response and the impact on the amplification of initial imperfections and lateral deformations. The inverse Southwell procedure is based on the pioneering, combined analytical and experimental work of Southwell (Roorda 1965 and 1967), which was developed to allow a structural analyst to estimate the buckling load amplitude for a given structure, load distribution, and load amplitude. In Southwell's procedure the buckling load is based on extrapolations from measured deformations under a specified load distribution but with an amplitude that is considerably below the critical level for the structure. Conversely, the inverse of this procedure can be used to determine the prebuckling deformation in the structure if the buckling load can be determined from some other procedure. This inverse process based on the Southwell procedure, combined with a simple eigenvalue approach to independently determine the critical buckling load amplitude, allows the calculation of deformations for induced load distributions or initial imperfections or both. Appendix A gives the eigenvalue approach to determine the critical bifurcation load for a single-membrane design. A similar approach can be used for double-membrane designs, but a more comprehensive method based on variational procedures is presented in Murphy (1985) for a range of design configurations. 
Discussion and use of the Southwell approach in this report are limited to situations where the deformations are fairly small. This approach will be shown to be adequate for our purposes here as this restriction is consistent with the analysis presented in Murphy and Sallis (1984) and also consistent with well-designed membrane/frame combinations in which the initial imperfections must be small. The limitation is also true for any deformations that occur subsequent to the application of wind-induced lateral pressure or weight, or tension loading of the module, to ensure adequate optical quality of the structural system.

We can state the essence of linear Southwell analysis in the following manner. If we let the ordinate of a curve (see Figure 3-1, where a simple column structure subjected to an axial load $P$, is illustrated) be the characteristic deformation divided by the applied load on the structure and let the abscissa be the characteristic deflection $\delta$ of the structure, which has an initial deflection $\delta_{0}$ when $P=0$. Then the slope of the curve so defined, in the linear analysis, is the inverse of the critical load for the structure. Thus, if one can predict either experimentally or with an accurate numerical structural code the deflection of a particular structure for at least two load levels, then one can establish a linear approximation of the critical bifurcation load for the structure with the assumed loading distribution. Conversely, if the buckling load is known, as from an eigenvalue approach, then the slope of the Southwell curve is determined, and only one load deformation point is needed to define the prebuckling load deformation path. Note that in Figure 3-1 the deformation of the beam that is described is essentially normal to the application direction of the load and not the deformation under the load, which will depend nonlinearly on the lateral deformation.

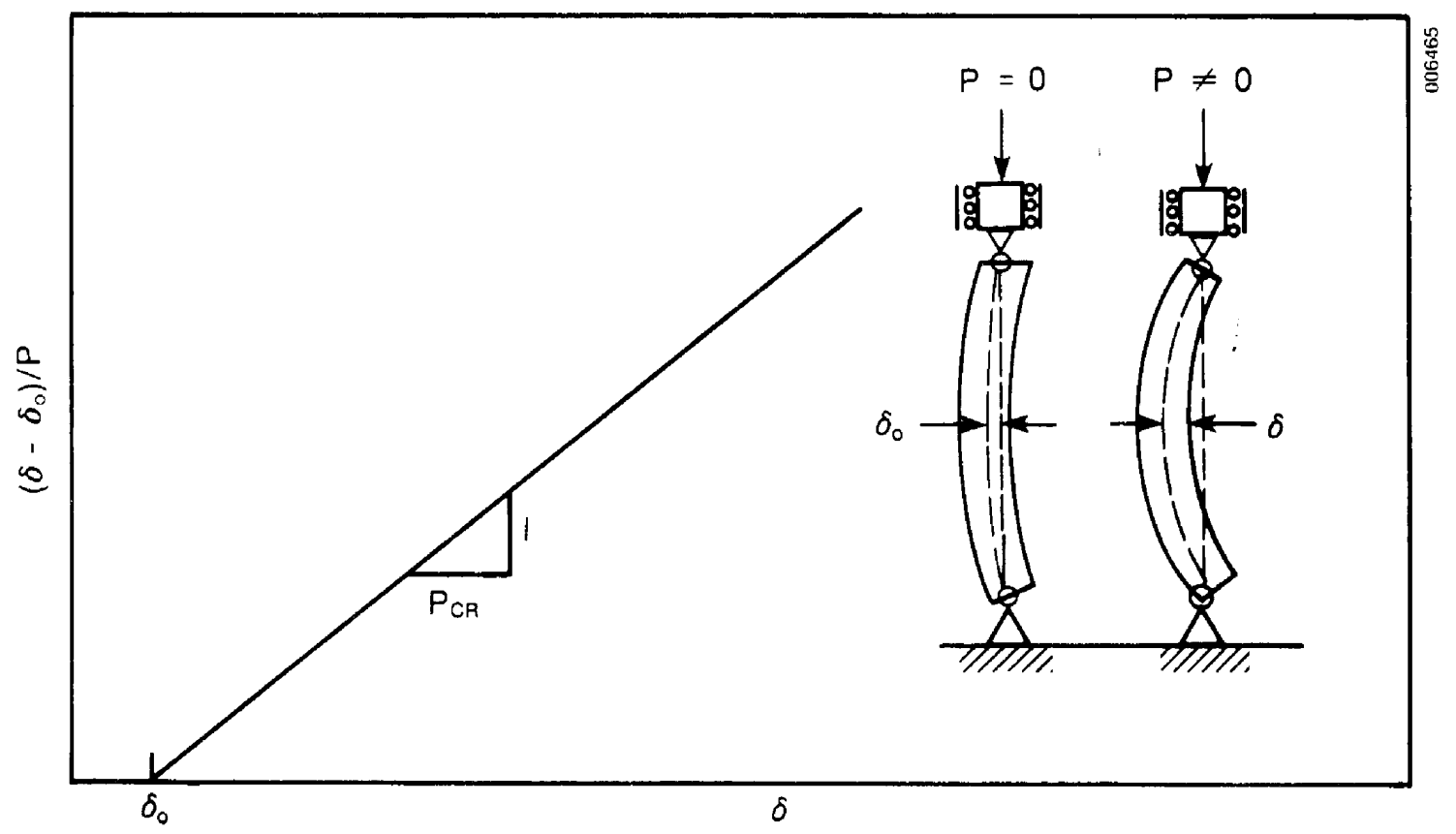

Figure 3-1. Linear Southwell Plot for a Simple Beam Structure 
The bifurcation load mentioned above, for a given structure and loading pattern, is the load at which more than one equilibrium configuration can exist. A state of neutral equilibrium is said to exist at the bifurcation load. If the structure has initial imperfections in a mode shape similar to the buckled shape, then in general a true bifurcation of equilibrium will not be observed but rather the deformation increases and becomes unbounded as the critical bifurcation load, for the corresponding perfect structure, is approached. A similar situation occurs if the prebuckling deformation contributes to the buckled shape.

Note that the linear Southwell approach can give little insight into the postbuckling description of the load deformation process other than the anticipated mode of deformation. In general, the Southwell lines may not be linear as is assumed with moderately small deflection theory, and as implied in Figure 3-1, but may be curved, particularly near the critical bifurcation point. The Southwell analyses described here and later implicitly assume that one can ignore nonlinearities in the Southwell lines at least to a first approximation. This turns out to be a very good assumption for load levels below about $60 \%-70 \%$ of the bifurcation load in the case studied.

\subsection{Stability Considerations for Single-Membrane Concepts}

Consider the buckling of a free-standing circular ring or a circular ring with a single membrane attached to it that is coincident with the plane passing through the frame torsional center, as illustrated in Figure 3-2a. The ring, to be discussed extensively later, is assumed to have a radius of $R=5.0 \mathrm{~m}$, a flexural rigidity of $\mathrm{EI}_{\mathrm{y}}=1.77 \times 10^{6} \mathrm{~N} \cdot \mathrm{m}^{2}$, and a torsional rigidity of $\mathrm{GK}=0.38 \times 10^{6} \mathrm{~N} \cdot \mathrm{m}^{2}$. The membrane is steel $0.254 \mathrm{~mm}$ (0.010 in.) thick, and the frame has an in-plane $E I_{x}=0.384 \times 10^{6} \mathrm{~N} \cdot \mathrm{m}^{2}$. This stretched-membrane frame is identical to the frame discussed and studied extensively in Murphy and Sallis (1984). Appendix A discusses the implications of attaching the membrane on the frame above or below its current assumed position. For the purposes of discussing the NASTRAN numerical results, we will assume that the ring or the ring/membrane combination will deform out-of-plane in the $n=2$ mode (i.e., the lowest fundamental buckling mode), and when an initial imperfection is introduced in the NASTRAN solution it is induced by four concentrated loads of equal magnitude (of amount $\mathrm{P}=222 \mathrm{~N}\left[\begin{array}{ll}50 & \mathrm{lb}\end{array}\right]$ ) but of alternating sign.* The alternating concentrated loads are placed on the ring at $90^{\circ}$ intervals in a direction that is normal to the plane of the ring as shown in Figure $3-2 b$.

In Appendix $\mathrm{C}$, we discuss briefly in-plane buckling and show that the corresponding critical membrane tension is considerably higher than that corresponding to out-of-plane buckling for the frame/membrane combination. Because of this we can in many cases consider the two buckling processes independently. The relatively high in-plane critical loads for a frame/membrane combination result from the higher frequency buckled shape, which is in turn caused by the membrane constraint. Thus, in general, for the membrane/frame combination to buckle in the radial direction the appropriate deformation mode number will be considerably higher than the $n=2$ fundamental mode for out-of-plane buckling. We have not investigated coupling between in-plane and outof-plane buckling modes, although we have seen no tendency for this to be a problem based on the NASTRAN analyses to date, where only fixed (infinite stiffness) supports are considered.

* This approach is computationally much more simple to implement in NASTRAN than introducing displacement imperfections. 


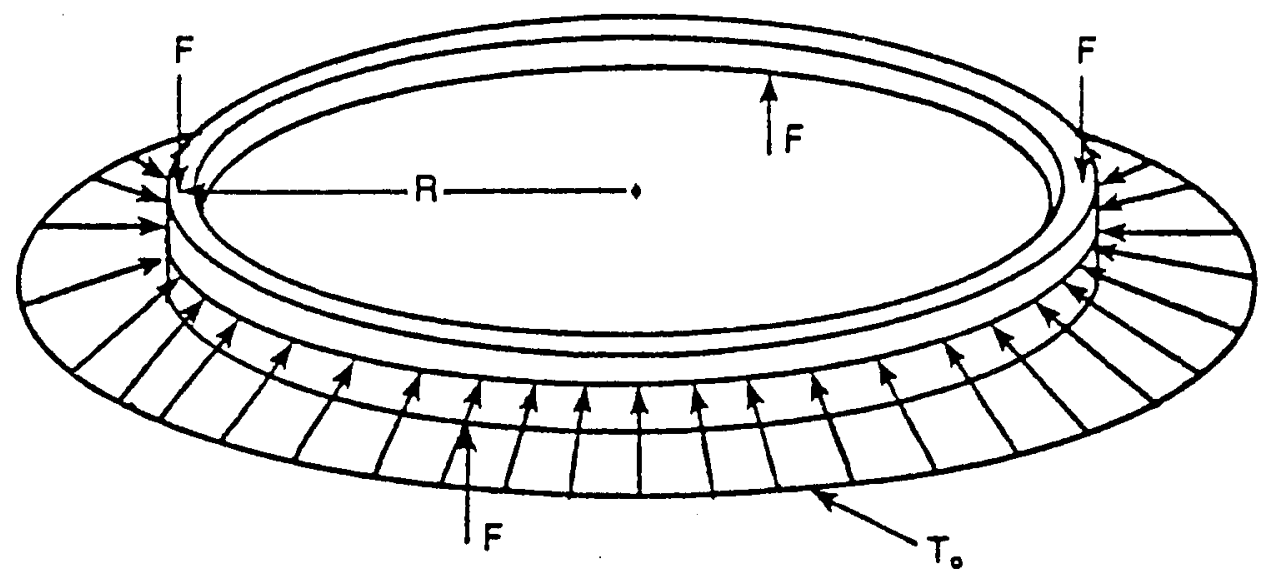

$\overline{\overline{\$}}$

a) Ring Irame only with radlally Inward llve load

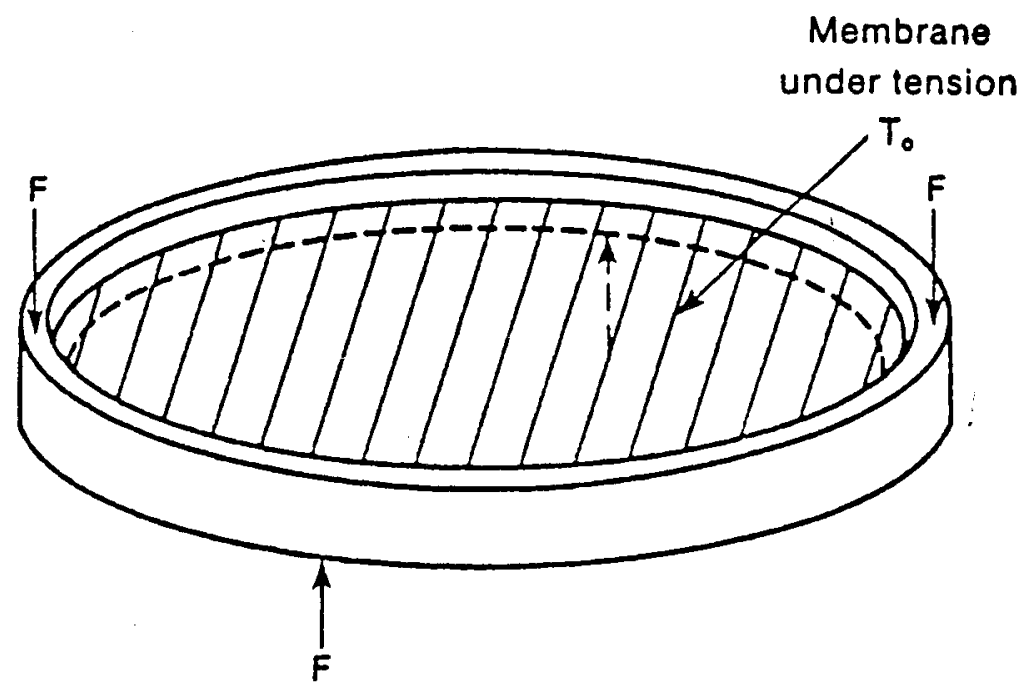

b) Ring frame with membrane allached and under lension $T_{0}$

Figure 3-2. Geometry and Loading on the Ring Frame with and without the Membrane Attached 
Predicted results for both a radial loaded ring without a membrane and for a ring/ membrane combination are presented in Figure 3-3. The peak lateral (out-of-plane) deflection, $v_{\max }$, of the frame, which occurs midway between the supports, is a function of total radially inward (or compressive) frame load illustrated in Figure 3-2. The $n=2$ mode of out-of-plane deformation will result, as can be inferred by the loading described in Figure 3-2. When a membrane is present, the compressive frame load corresponds to the load that the membrane induces in the ring. When no membrane is present, we assume that the compressive load on the ring is induced by a uniform circumferential line load (dead load) in the radially inward direction that is parallel to the original membrane plane. The data in Figure 3-3 illustrate a number of stability measures. The first vertical line at $14,700 \mathrm{~N} / \mathrm{m}$ corresponds to the bifurcation buckling condition for the frame unconstrained by the membrane as predicted by both NASTRAN (Schaeffer 1979) and the classical solution developed by Timoshenko (1961), respectively. As noted above, the critical bifurcation load is the load at which more than one equilibrium configuration can exist for an initially perfect ring. This vertical line indicates a bifurcation of equilibrium at a constant tension load and implies a catastrophic collapse of the ring. Moving now to the first convex upward curve at the left in Figure 3-3, the out-of-plane ring deformation path for an initially imperfect ring subject to increasing tension loads is illustrated. The crosses indicate specific NASTRAN-predicted solutions for peak lateral deflection versus tension, and the dashed curve represents the prediction from the Southwell procedure based on extrapolation from the first two discrete load/deformation predictions obtained using NASTRAN. As noted above, the Southwell solution extrapolates information based on an initial imperfection of the structure and the initial slope of the load-deformation curve and uses this information to describe the load/deformation trajectory and the critical load; this extrapolation also agrees extremely well (within $0.5 \%$ ) with the next two NASTRAN predictions. Further, an ultimate collapse load for the frame results, which is quite close to either the solution for the initially perfect ring as predicted by Timoshenko (1961) or NASTRAN.

The remainder of the discussion relating to Figure 3-3 corresponds to the $\mathrm{ring} / \mathrm{membrane}$ combination. A solid curve passes through the triangles, which represent discrete NASTRAN-predicted maximum out-of-plane deformations for the initially imperfect ring/membrane combination. The critical bifurcation load, calculated with NASTRAN and linear eigenvalue theory, is $29,416 \mathrm{~N} / \mathrm{m}$. The dashed curve above the solid curve represents the predicted deformation path using the Southwell procedure and the first two NASTRAN predictions for the assumed loading condition. This Southwell path follows the NASTRAN solution quite closely until a load of about $22,000 \mathrm{~N} / \mathrm{m}(72 \%$ of the bifurcation load) is reached.

The prediction for the bifurcation load, using classical linear eigenvalue theory and where initial imperfections are assumed to be zero, is derived from the governing membrane/frame deformation equations (see Appendix A). For the $n=2$ mode $\mathrm{ring} / \mathrm{membrane}$ combination, the classical eigenvalue bifurcation load at $T_{0}=29,416 \mathrm{~N} / \mathrm{m}$ is exactly two times the classical eigenvalue bifurcation (Timoshenko 1961) load of $14,708 \mathrm{~N} / \mathrm{m}$, which corresponds to the ring only. In fact, Appendix A shows that for any mode shape the critical bifurcation load for the frame ring/membrane combination $\left[T(n)_{C ; R / M}\right]$ is related to the critical bifurcation load of the frame ring only $\left[T(n){ }_{C ; R}\right]$ by the expression

$$
T(n)_{C ; R / M}=T(n)_{C ; R} /(1-1 / n)
$$




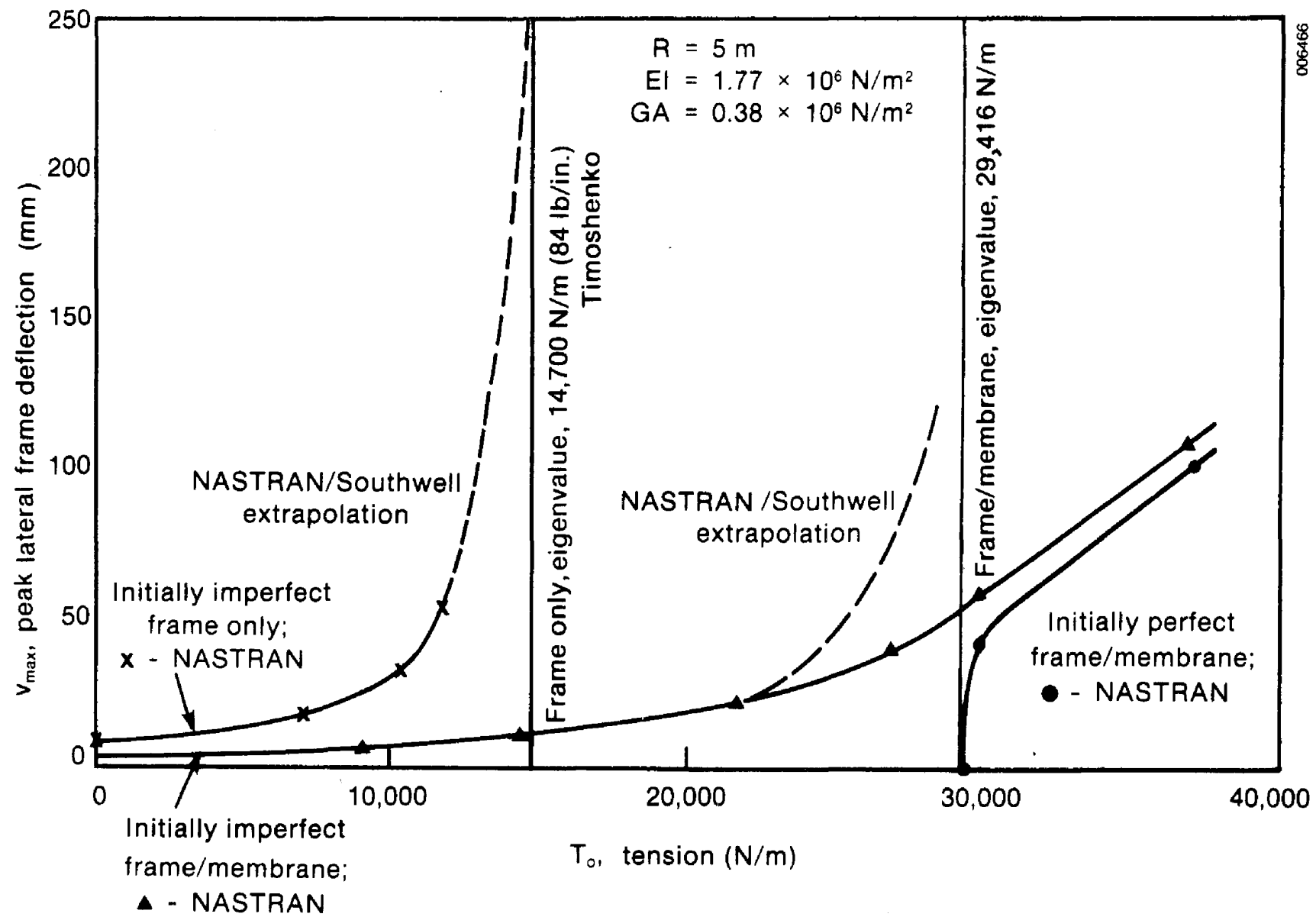

Figure 3-3. Peak Out-of-Plane Frame Deflection versus Radial Tension Load for a Number of Frame/Single-Membrane Combinations

where $n$ corresponds to the buckling mode number corresponding to the number of waves around the circumference of the ring.

Although the eigenvalue and linear Southwell approaches are helpful in providing information about the critical bifurcation load and the prebuckling deformation, the postbuckling behavior is not described. Figure 3-3 shows that the NASTRAN solution does not predict a catastrophic collapse of the membrane frame assembly as is implied by the linear analysis but rather predicts increasingly large and nonlinear deformations as the membrane tension increases, up to and beyond the critical bifurcation load. The membrane also offers restraint (i.e., increased system stiffness) to collapse and, therefore, in a sense protects the assembly from a catastrophic failure. This restraint occurs because the membrane provides a self-limiting feature whereby large out-of-plane deformations are inhibited by restoring forces from the membrane.

The concave downward curve to the far right in Figure 3-3 shows the NASTRAN predictions for an initially perfect ring/membrane combination. In this combination, there are no out-of-plane loads applied to cause initial imperfections. We calculated points on this concave curve in the following manner. First, we placed a set of out-of-plane distortion loads (as in Figure 3-2) on the ring and applied the tension. Then we reduced the out-of- 
plane loads to zero and allowed the ring/membrane combination to seek its preferred equilibrium shape. Hence the results show that for loads below $29,400 \mathrm{~N} / \mathrm{m}$, the $\mathrm{ring} / \mathrm{membrane}$ returns to its initial undeformed configuration when one removes the outof-plane loads. When we applied a tension load above $29,400 \mathrm{~N} / \mathrm{m}$ and removed the outof-plane loads, the compressive tension loads, whose peak amplitude as a function of tension is defined by the concave curve, maintained a distorted configuration in the $n=$ 2 mode. As tensions increase beyond the bifurcation load, the NASTRAN solution for the imperfect ring frame and the perfect ring frame combination, respectively, are asymptotically close at high tensions and differ only by a few millimeters of deflection for the membrane/frame combination studied and for any given membrane tension level. Another feature of these two curves is that if we look at the point at which a predicted bifurcation of equilibrium will occur for the initially perfect system (at about $29,400 \mathrm{~N} / \mathrm{m}$ ), the resulting critical load is quite close to the eigenvalue solution $(29,416 \mathrm{~N} / \mathrm{m})$ for the ring/membrane combination (Eq. 3-1). Thus NASTRAN and the simple eigenvalue approach from Appendix A and Eq.3-1 give essentially the same answer for the critical bifurcation load.

We should emphasize that the predicted load deformation trajectories illustrated in the preceding curve correspond to the $n=2$ mode. This $n=2$ configuration, where the concentrated loads induce a simulated initial imperfection, simulates a situation in which the membrane/frame combination will deform before one installs the frame/membrane combination on the supports. Hence this $n=2$ mode will superimpose on other deformation modes that are caused by external pressure, weight loads, and constraints. The $n=2$ mode, which in addition to being the fundamental (most likely to occur because of the smallest required load) buckling mode, is the mode that would most probably correspond to the largest imperfection and hence leads to the largest amplification of an initial imperfection. A similar set of corresponding curves with different amplitudes and at corresponding higher tension loads would result if the deformation were constrained to follow the $n=3$ mode (i.e., as in a situation where the module is uniformly pressure loaded and where three supports are present like those described in Murphy and Sallis [1984]).

Figure 3-4 illustrates another stability analysis. In this analysis, a membrane under a tension load, which is larger than the buckling load of the ring alone $(14,700 \mathrm{~N} / \mathrm{m}$ without the membrane) but smaller than the bifurcation load $(29,400 \mathrm{~N} / \mathrm{m})$ corresponding to the ring/membrane combination, is applied to the frame. Again, the $n=2$ mode of deformation is investigated in this figure, and the membrane deflection is plotted as a function of radial coordinate along lines of constant circumferential coordinate $(\theta)$. Note that the deformations described in Figure 3-4 are no longer small, and the assumptions relating to linearity and linear Southwell analysis will no longer hold.

Figure 3-4 illustrates three lateral loading cases for the ring/membrane combination that has the same geometric properties as the combinations described in Figure 3-3. The lowest set of solid curves (A) corresponds to the case where an initial imperfection is introduced with four concentrated loads of $222 \mathrm{~N}(50 \mathrm{lb})$ each, as described in Figure 3-2b. The upper set of solid curves (B) corresponds to a uniform pressure loading (90 $\mathrm{Pa}$ ) on the membrane. Finally the upper set of dashed curves $(\mathrm{C})$ corresponds to the combined loading that is assumed for cases $A$ and $B$ (i.e., concentrated frame loads and a pressure load).

Figure 3-4 illustrates two major points. First, the solutions for the two loading cases (A and $C$ ) are not linearly additive. This is verified by noting, for any given radius and circumferential coordinate (e.g., $\theta=0$ and $r=5.0$ ), the difference between the top dashed 


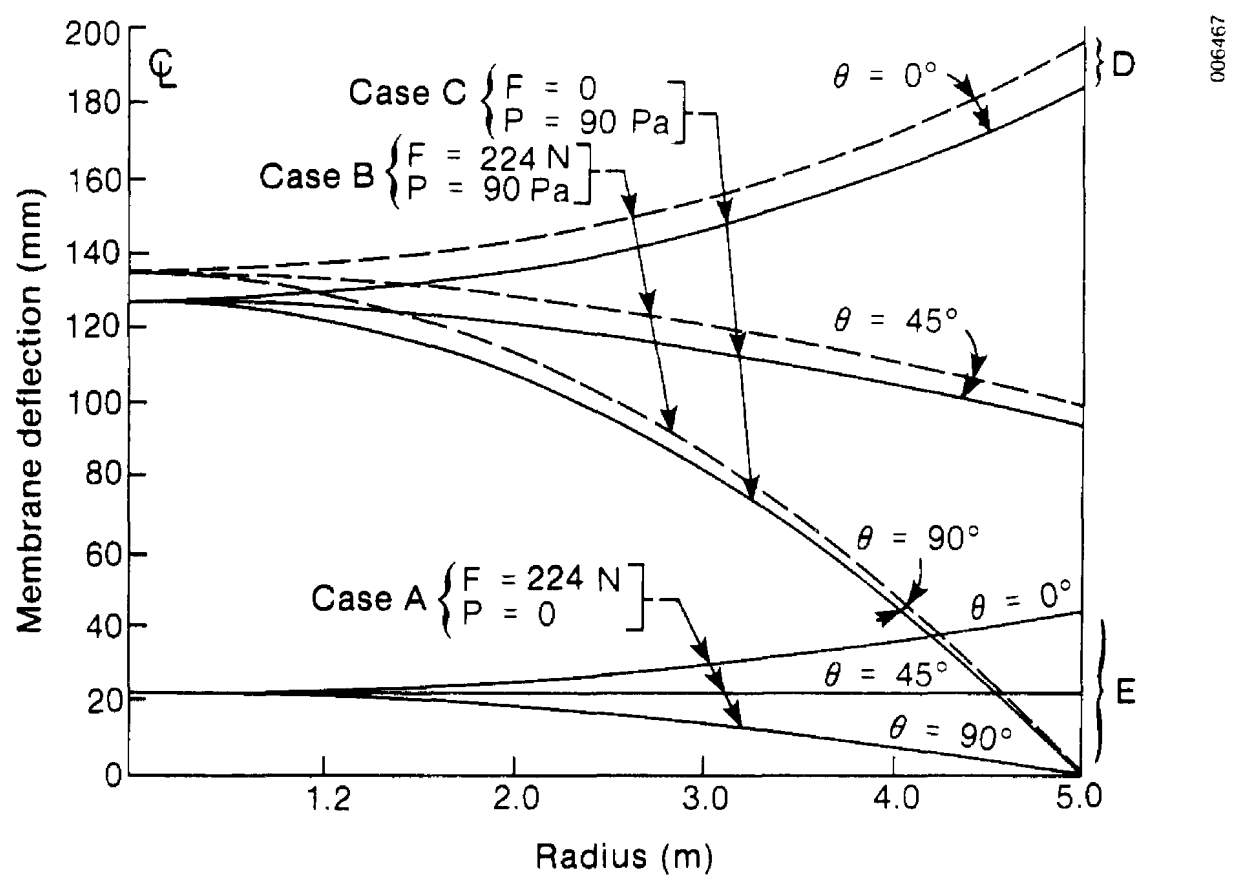

Figure 3-4. Single-Membrane Deformation versus Radius at Several Circumferential Sections Corresponding to the $\mathrm{n}=2$ Frame Deformation Mode

and top solid curve (marked D) and comparing it with the difference between the solid curve for case $A$ and the abscissa (marked E). If linear superposition were applicable, then the solution for pressure loading only could be linearly added to the solution for the concentrated load only and these two relative differences would be equal.

The second major point illustrated is that when the tension load on the ring is greater than the buckling load corresponding to the ring only, the deformation of the frame will exceed the membrane deformation. That is, the membrane deformation must remain within the two extremes of the frame deformation when the ring-only buckling load is exceeded. This is because the frame no longer is able to carry the lateral load by itself, and the membrane must assist the frame in carrying the load; to do so the frame must deflect out away from the membrane. If the membrane did deflect outside the frame cross section at any location, the frame must support the membrane, which it cannot do. Thus, when the tension on the ring is greater than the ring-only buckling load, the frame must distort significantly to allow the frame and membrane to work effectively together. Conversely, when the membrane tension load is below the frame's buckling load, the frame tends to support the membrane by itself. Thus, for a wind-induced pressure or weight load on the membrane, the frame deflects less (more) than the membrane for tensions below (above) the frame-only critical load. Hence, the design tension for the single-membrane concept should be kept below the frame-only critical load level. This is especially true when three frame supports are used and it is pussible that the $n=2$ mode of deformation will not be restrained. Such an approach, however, is not required for the double-membrane design since the in-place stiffness of the membrane is coupled into the frame directly. 


\subsection{Stability Considerations for Double-Membrane Concepts}

All the double-membrane examples that were analyzed and are discussed later used the same frame as the single-membrane example discussed previously. The double membranes were also assumed to have radial attachments (see Figure 2-3). These analyses again considered the $n=2$ mode shape with four 224-N (50-lb), evenly spaced out-of-plane loads to set the initial deflections (see Figure 3-2). Figure 3-5 shows the out-of-plane deflection results and some of the results for the single-membrane cases previously shown in Figure 3-3. The first two sets of curves on the left correspond to the frame-only and the single membrane/frame combination cases. The curve with the highest tension levels (farthest to the right) has two $0.254-\mathrm{mm}(0.010-\mathrm{in}$.) steel membranes. The curve second from the right represents a case in which the thickness of the two membranes was reduced to $0.0587 \mathrm{~mm}(0.00231$ in.) to demonstrate the effect of reducing the stiffness of the membranes.* Thus, for these two examples, the addition of the second membrane increases the buckling load significantly above that of a single membrane. For the membranes, $0.254 \mathrm{~mm}$ (0.010 in.) thick, this relative increase is 2.83 times the single-membrane critical buckling load, and for the reduced membrane stiffness example the corresponding factor is 1.79. Note that the relative out-of-plane stiffness as indicated by the deflections at zero tension are essentially proportional to the buckling loads for the various cases. That is, the ratios of the zero tension deflections indicate that the 0.01 double-membrane system is 2.78 times stiffer than the single-membrane system and, correspondingly, the double membrane $0.0587 \mathrm{~mm}$ $(0.00231$ in.) thick is 1.78 times stiffer.** Figure $3-5$ also shows that the level of deformations and the tension range over which these deformations remain small are greatly extended beyond the region associated with the single-membrane concept and frame-only concept.

The two vertical lines at about 52,000 and $82,000 \mathrm{~N} / \mathrm{m}$ tension levels, respectively, in Figure 3-5 indicate the predicted critical buckling load for the double-membrane concepts. In Figure 3-5, a circled asterisk on the abscissa indicates for each of the cases the critical bifurcation tension load for initially perfect double-membrane structures, calculated by simple eigenvalue theory. Note that the NASTRAN-predicted critical tension loads and the simple eigenvalue bifurcation tension loads are in extremely good agreement. For the case shown, the NASTRAN predictions for the critical buckling load are based on deformations and tension levels that are quite close to the actual respective critical tension level. Hence, the extrapolation used by NASTRAN procedure should be quite accurate.

Figure 3-5 shows several interesting features not previously anticipated. Specifically, considering the load deformation trajectories for the double-membrane concepts, the transition region going from small to large deformations is more abrupt than the corresponding region for the single-membrane design. In fact, for the double-membrane case, the load deformation trajectory is similar to the trajectory corresponding to the frame-

* From a practical perspective the operational tensions will result in unacceptably high stresses in the membrane. However, this artifice illustrates the effect of low-membrane stiffness that occurs when polymer membranes are considered.

* Note that for double-membrane modules unacceptable load reversals in the membrane can occur at very low initial tension levels as is discussed in Section 2.8 . Hence the zerotension points, described in Figure 3-5 and later in Figure 3-6, are actually determined by extrapolation from low-tension data points where no load reversal was experienced. 


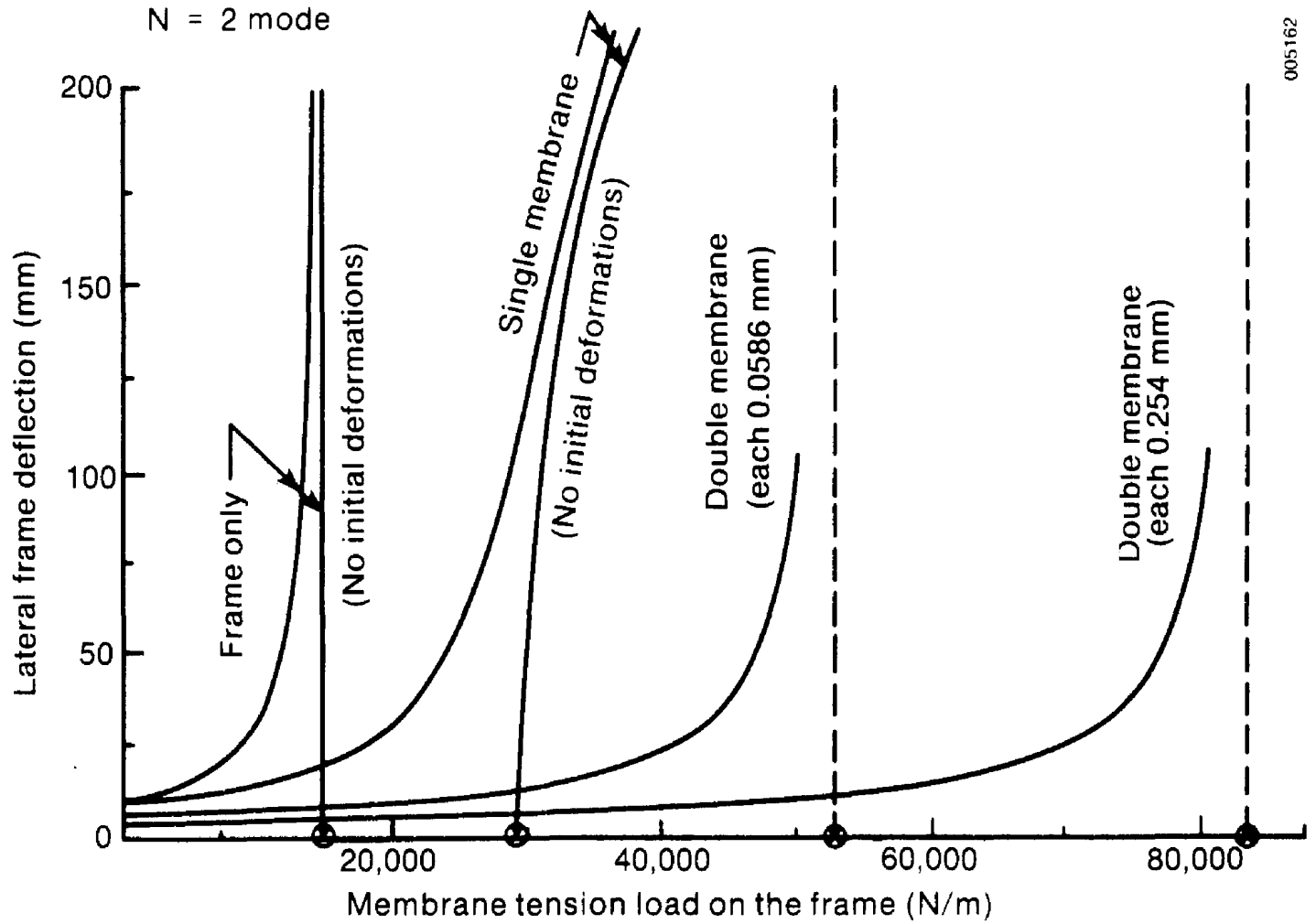

Figure 3-5. Peak Out-of-Plane Deflection as a Function of Tension for Several Single-and Double-Membrane Designs Corresponding to the $n=2$ Frame Deformation Mode

only case where a catastrophic collapse occurs at the critical bifurcation tension load. We do not know at this time whether the abrupt critical portion of the deformation trajectory for the double-membrane cases represents a true singularity in the stiffness matrix, such as might occur when the membrane locally unloads and attempts to sustain a compressive load, or if in fact the deformation trajectories would bend over (concave with respect to the abscissa) and avoid a catastrophic collapse as occurs with the singlemembrane design. Further study is needed in this area if postbuckling behavior is of interest. However, the simple eigenvalue approach and the extrapolation based on the NASTRAN analysis for the critical bifurcation level are in good agreement. Hence, the prebuckling deformations and the regions prior to the point where large deformations become dominant appear to be accurately predicted.

\subsection{Stability and the Impact on Initial Imperfections}

The curves in Figure 3-5 also indicate the relative out-of-plane deflections for various designs in which a given out-of-plane load causes the initial deflection. Note that the out-of-plane deflection for the single-membrane case is the same as the initial deflection corresponding to the frame-only case at low-tension levels, whereas for the doublemembrane cases the deflections at low tensions are less and depend on the membrane 
stiffness. This is because the out-of-plane buckling load (made by the addition of a single membrane) improves as a result of the direction changes of the tension load; therefore the membrane does not modify the out-of-plane stiffness of the single-membrane design at low tensions. For the double-membrane case, the additional improvement is the result of an increase in the out-of-plane stiffness caused by the torsional coupling as discussed previously in conjunction with Figures 2-7 through 2-9. This results in a more significant improvement (reduction) in the out-of-plane deflections at low tensions for the doublemembrane than for the single-membrane case when the initial deflections (i.e., at zero tension) are caused by a wind-induced pressure or weight load. However, when considering the effect of manufacturing errors, this trend is not as significant since the initial deflections are fixed. To demonstrate this point, we derived and present in Figure 3-6 a set of curves for the two double-membrane designs starting with the initial deflection of the single-membrane design, which is described in Figure 3-5. Now note that all the designs start out at the same out-of-plane deflection and improve less dramatically in the tension range where an actual design may operate. For example, consider the various design cases at $17,500 \mathrm{~N} / \mathrm{m}$ tension. The benefit in decreasing the expected amplification of initial imperfections by selecting a double-membrane design rather than a single-membrane design is about one-half as great as the corresponding benefit in supporting an applied load. Selecting stiff membranes rather than flexible membranes yields even less additional improvement. Therefore, we expect that the greatest benefit of the double-membrane design is to reduce the deflections caused by wind-induced pressure and weight loads.

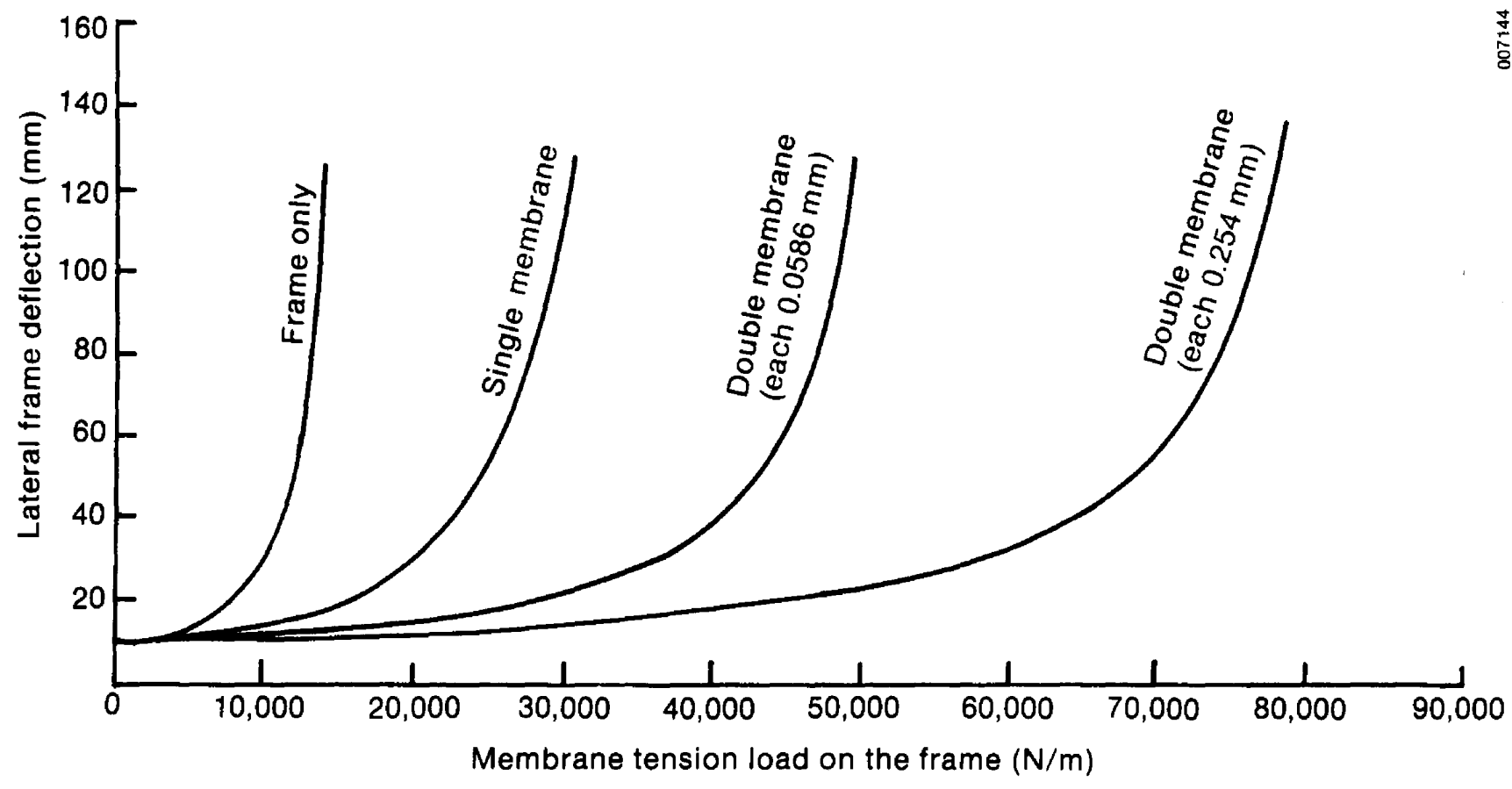

Figure 3-6. Out-of-Plane Deflection Comparison versus Tension for a Manufacturing Type (Fixed), $\mathbf{n}=2$ Mode, Initial Frame Deflection 
In Appendix D, we present a more extensive discussion of initial imperfections and show that the amplification factor is a function of the tension ratio, defined as the operating tension in the membrane divided by the critical bifurcation tension. The use of this amplification factor also leads to reasonable approximate design models to predict the lateral response of stretched-membrane modules. The analysis presented in Appendix D is directed at single-membrane designs but can be extended to double-membrane designs as well. One important feature is that the amplification of initial imperfections depends on the shape of the imperfections as well as the amplitude. Thus the amplification depends on the mode shape of the imperfection and the tension level as well as the nearness of the tension level to the critical tension level corresponding to the mode shape of interest. 
TR -2338

\subsection{DESIGN IMPLICATIONS AND RECOMMENDED AREAS FOR FURTHER INVESTIGATION}

\subsection{Implications for the Design of Stretched-Membrane Modules}

The single most important structural design parameter for the stretched-membrane module is the out-of-plane flexural rigidity of the frame. The frame torsional rigidity is much less important when double membranes, which have reasonably high stiffness, are used and when the attachment of the membrane to the frame is relatively stiff. The torsional rigidity, however, is very important when a single membrane is used, although it is not quite as dominant a factor as the flexural rigidity. Torsional rigidity also becomes more important when low-stiffness membranes or low stiffness attachments or both are used in double-membrane designs. Such a situation may arise with polymer membranes that typically have very low moduli, or when a soft attachment of the membrane to the frame such as with the hydraulic/bladder approach as described in Murphy (1983) is used.

The attachment approach as noted previously can heavily influence the module performance since it represents the structural interface between the frame and the membranes and thus significantly controls* the effective structural system stiffness. This is especially true for the double-membrane designs. Thus, attachments affect the level at which the membrane can enhance the bending stiffness of the frame through roll resistance and through the direct flange bending stiffness effect described previously. Furthermore, the attachment limits the ability of the membrane to add stability margin to the module structure and, thus, indirectly controls the amplification of initial imperfections and load-induced deformations with double-membrane concepts. When using stiff membranes, hard bonds or welded attachments relative to the radial-only constraint attachment can add $25 \%$ or more out-of-plane stiffness for some single-membrane designs and $40 \%$ or more out-of-plane stiffness for some double-membrane designs.

The double-membrane reflector module is generally structurally superior to the singlemembrane concept, since this approach most effectively couples the membrane stiffness to the frame and, therefore, uses the membrane material to pick up part of the additional applied loads. However, care must be exercised to ensure that no localized unloading of the membrane will occur. Most of the structural benefit of the doublemembrane approach accrues by directly enhancing the roll resistance of the frame. For instance, the double-membrane concept can more than double the effective out-of-plane bending stiffness of the module compared to a single-membrane design. Further, from a weight perspective, the double-membrane concept offers a more efficient structural stiffening effect than a corresponding increase in flexural rigidity. Moreover, the double membrane can add significant stability margin to the module stability and thereby inhibit the amplification of initial imperfections as well as the out-of-plane deformations caused by wind-induced pressure and weight loading. Although the single-membrane designs should probably be limited to tension levels that are well below the ring-only buckling load for reasons discussed previously, no such limitations are readily apparent in the double-membrane designs. From a design and fabrication perspective, the doublemembrane concept makes it possible to use an open cross section (e.g., a standard $\mathrm{C}$-section) since low torsional rigidity is permitted. This allows not only some savings in

* See also Appendix $C$ where a simple model describing the coupling between the attachment and the membrane is discussed. 
weight but also potential ease of attachment and handling when affixing the membrane to the frame.

Studying the stability effects of the membrane/frame structure has led to several insights, even though we know that the early designs will have tension levels that are well below the critical stability limits. The previous analysis indicates that the nearness to the critical tension level dictates the amplifications of initial imperfections in the frame and affects the degree to which large nonlinear deformations begin to dominate the deformation process. The analysis also demonstrated that the membrane adds significantly to the overall stability of the membrane/frame configuration so that the critical bifurcation load is significantly raised above that of the frame-only configuration. The single-membrane design does not appear to have a catastrophic collapse mechanism though amplification of lateral deformations and initial imperfections can proceed to undesirable levels if a careful design procedure is not followed. Further, for the singlemembrane system, the critical bifurcation tension level is approximately double that of the frame only. For the double-membrane system the critical bifurcation tension level can be a factor of five or six times that of the frame only depending on the membrane stiffness and the stiffness of the attachment approach used.

Although we have not studied the effect of different material stiffnesses and densities thoroughly, the results to date indicate that the lighter materials may offer a significant cost and performance advantage. This is because the frame deformation is primarily dominated by weight effects. In the cases investigated, an aluminum frame/membrane combination of the double-membrane design concept of fered approximately a three-toone weight advantage for the same performance as a steel design.* Although the cost of aluminum is approximately one-third that of steel, approximately one-third the weight of aluminum material is required for the design. In addition, significant benefits in handling, shipping, and fabrication might accrue with this lower weight.

One major design consideration for stretched-membrane modules is the initial tension in the membranes. A number of factors will control the tension design criteria. Design factors that will favor lower tension levels include limitations on the membrane material thickness and strength, the frame weight, and the tendency of increased tensions to amplify the initial out-of-plane frame deformations. The design limitations caused by the properties of the membrane materials coupled with the available material thickness are a straightforward consideration, though one must exercise care to allow a sufficient safety margin for stress concentrations near attachments and welds.

The lower tensions lead to lower stiffness requirements, and lesser potential gross and local frame-buckling problems while resulting in lower overall frame weight. Frame weight can typically account for more than half of the concentrator weight. Similarly, since the amplification of initial imperfections is closely related to frame stability, as shown above, lower initial tensions can reduce the corresponding amplifications to lower levels for a given frame design, or allow for a lighter frame design for a specified maximum permissible frame deformation.

Higher initial membrane tensions can greatly abate the potential wind-induced flutter problem and the attendant fatigue problem. Also, higher initial membrane tensions can significantly lessen the potential problems associated with nonuniform wind-induced pressure loading and in-plane loading problems, as well as greatly reduce the potential

* Note that we assumed the same membrane thickness in the above analysis. 
problems associated with load reversal in double-membrane concepts. Further higher initial tensions reduce the required axisymmetric optical surface control caused by wind loading and, in the case of double-membrane designs using a dynamic vacuum control, can significantly lessen the required parasitic power since the amplitude of the breathing mode can be significantly lessened.

Another benefit of higher initial tensions is that the effect of nonuniform tension states caused by large axisymmetric deformations and diaphragm stretching can be greatly lessened.* Such nonuniform tension states result in nonuniform membrane surface curvatures and thus optical distortions. With high initial tensions both the absolute tension increases for a given pressure load and the variations in tension relative to the average membrane tension are less than will occur for low initial tensions; thus the potential optical distortions will be decreased significantly. Note that for most of the cases considered in this report, diaphragm stretching is not a significant problem; however, in designs with relatively high-pressure loadings and low tensions, diaphragm stretching can become a significant problem. Finally, if diaphragm stretching is significant additional tension loads on the frame will result. With higher initial tensions, the frame will experience much lower relative tension increases on the frame.

\subsection{Structural Response Issues Requiring Further Investigation}

The preceding analysis and discussion provided many insights into the structural response and design of stretched-membrane reflector modules. We addressed many of the major structural and optical response issues with both simple and more rigorous analysis methods (i.e., NASTRAN). Researchers are currently undertaking prototype testing to confirm many of these analyses and the insights derived, and initial experimental results are documented in Wood (1986a and 1986b). Numerous other issues have not been studied or have not been studied sufficiently and will take on increased significance as the designs become more refined, and as we approach the optimum reflector module configuration. Ultimately, these secondary issues may drive the fine details of the design.

In the first category of issues that we have not studied are the problems of in-plane weight and support effects, which will lead to completely nonsymmetric loading and deformation conditions between the supports, and radial thrust or hard point effects caused by the strut supports that connect the frame to the center column post. The attachment of the frame to the support struts may also lead to concentrated moments at this interface, which we have not studied. In addition, researchers have not assessed the effects of nonuniform pressure loads nor have they considered local frame-stability issues such as wall "crippling." Furthermore, researchers have not considered a sufficient range of radial stiffness effects of the frame in the analysis to date. This last issue may be particularly important when we consider soft membrane and soft attachment concepts because of the potential coupling with the in-plane buckling that we have studied only briefly. Also, the radial stiffness effect will come into play when localized membrane unloading becomes a concern.

Included in the second category of issues that we have only partially addressed are the effects of highly compliant membranes, low stiffness attachments, and the in-plane buckling response of the frame using these concepts. In addition, the amplification of

* See Appendix B for an approximate analytical description of the effect. A much more retailed description of this effert is given in a for thcoming nublication (Murphy). 
initial imperfections corresponding to different modal shapes, which are amplified at different levels according to the tension in the membrane, have not been studied sufficiently. Of particular interest is the manner in which deformation induced by external loads couples with initial imperfections (we have shown in previous discussions that at high tension levels, deformations induced by different loadings cannot be linearly superimposed). Furthermore, we have not sufficiently studied the use of more than three supports to straighten initially imperfect modules and to also prevent troublesome modes of deformation.

Another issue of particular importance, which needs more study, is the potential unloading of membranes as the frame rolls and reduces the tension load in the membrane. This problem emerged in some of the sensitivity studies of double-membrane designs where the tendency to locally unload the membrane was predicted to occur for some designs (especially with low initial tensions and low flexural rigidities). Such a situation is not acceptable in real designs because the membrane will locally buckle or "crinkle" upon load reversal. We believe that insights into the problem can be attained with correct analysis methods. Also, more analysis in the following areas would help us arrive at an optimal design: more extensive trade-offs and variations considering different materials along with their associated stiffnesses and weights; differences in frame radii; and the impact of support strut design, including the associated weight and material requirements.

Last, the problem of the dynamic response of the stretched-membrane module, including the gross structural response of the frame/membrane combination and local dynamic flutter conditions in the membrane, has not received sufficient attention to date. These dynamic issues are the subject of ongoing study. The gross structural dynamic response investigations, which are focusing on the critical vibration frequency corresponding to the membrane/frame combination, are being carried out at the University of Nebraska. Further, SERI currently has a contract with Colorado State University to study the fatigue and attendant flutter conditions that may exist in membranes because of transient wind-loading conditions in the field. The potential flutter problem is manifested by traveling surface waves and the reflection of these transient waves from hard points where the membrane is attached. 


\subsection{CONCLUSIONS}

The work provided in this document addresses a fairly broad range of structural response issues corresponding to stretched-membrane modules under various loading environments. Although the information presented here does not represent a complete picture of the response of the stretched membrane, it does represent a significant step in that direction. We believe that the trends presented here will help to effectively guide the design process and to build adequate but not necessarily optimal designs. Clearly, more work is needed to extend the knowledge base required to build optimally designed stretched-membrane modules.

Based on the above analysis, we have reached the following conclusions:

- Stretched-membrane/frame combinations respond quite differently to external loads than can be inferred by studying the decoupled frame and membrane independently. Thus the coupled membrane/frame problem should be considered to ensure an accurate description of its response.

- Nonlinear analysis methods are required to accurately predict the structural/optical response of stretched-membrane modules to the anticipated loading. The small strain, large deformation assumptions used in the approximate analyses appear to be adequate based on the agreement with the NASTRAN results.

- For the cases considered in this study, out-of-plane deformations of the membrane/ frame combinations were found to vary nearly linearly with load applied normal to the nominal plane of the membrane surface, while significant nonlinear response with membrane tension was found for the out-of-plane deformations.

- Out-of-plane frame flexural rigidity is important for both single- and doublemembrane concepts. Frame torsional rigidity is not as important an issue with doublemembrane concepts, but it becomes increasingly important when single-membrane concepts are used and when low-stiffness membranes or low-stiffness attachments of the membrane to the frame are used.

- The method of attaching the membrane to the frame can affect the performance of the module significantly, especially with double-membrane designs because the double membrane can both enhance the bending stiffness and the roll resistance of the frame.

- The double-membrane systems provide a very efficient structural system. From a weight perspective, the second membrane can, in fact, offer a more efficient structural stiffening effect than a corresponding increase in flexural rigidity with a singlemembrane concept. This occurs since the double-membrane design couples the membrane stiffness into the problem in a more effective way than is possible with singlemembrane designs. In addition, the second membrane adds significant stability margin to the reflector module and thus can inhibit the amplifications of initial imperfections as well as out-of-plane deformations caused by wind and weight loading.

- For the cases considered in this study, weight effects dominated out-of-plane deformations. Hence, the use of lower density frame and membrane materials were found to have the potential for significant performance improvement. Further, for these cases the performance improvement was found to be more responsive to decreases in weight than to a corresponding increase in material stiffness.

- There are significant potential benefits to using more than three frame supports, which must be traded off against the additional cost and complexity of using more than three 
supports. The potential benefits include dramatically reduced weight for the reflector module and the possibility of correcting for eliminating or abating the initial out-ofplane displacement imperfections and their subsequent amplification.

- Stability considerations are important when considering the amplification of both initial imperfections and the amplification of the deformations that result from external loading by wind and weight environments. Compared to the frame alone, the membrane/frame combination, whether of the single- or double-membrane design, will have a greatly enhanced stability margin under radially directed loads. This is true when considering both in-plane and out-of-plane frame deformations. Moreover, double-membrane concepts increase the stability margin significantly above that for a single-membrane design. Further, we demonstrate how the knowledge of the critical buckling tension level, which can be derived from the simple analyses presented herein, can result in some simple design tools. 


\subsection{REFERENCES}

Ariaratnam, S. T., 1961, "The Southwell Method for Predicting Critical Loads of Elastic Structures," Quarterly Journal of Mechanics and Applied Mathematics, Vol. 14, Part 2, p. 137.

Khoshalm, B. H., 1983, "50-kW Solar Membrane Concentrator," presented at the Solar Thermal Collectors Workshop (sponsored jointly by the Saudi Arabian National Center for Science and Technology and the United States Department of Energy), Sheraton Inn Lakewood, Lakewood, CO, 11-14 April 1983.

McGuiness, H., 1961, Solution of a Circular Ring Structural Problem, Tech. Rep. 32-178, Pasadena, CA: California Institute of Technology.

Meck, H. R., 1969, "Three-Dimensional Deformation and Buckling of a Circular Ring of Arbitrary Section," Journal of Engineering for Industry, Transactions of the ASME, pp. 266-272.

Murphy, L. M., 9 Nov. 1982, "A Non-Linear Membrane/Support Frame Load-Deformation Relationship for Design Trade-Offs," TN-253-004, Internal Memorandum, Solar Energy Research Institute, Golden, CO.

Murphy, L. M., May 1983, Technical and Cost Benefits of Lightweight, StretchedMembrane Heliostats, SERI/TR-253-1818, Golden, CO: Solar Energy Research Institute.

Murphy, L. M., Nov. 1985, A Variational Approach for Predicting the Load Deformation Response of a Double Stretched-Membrane Reflector Module, SERI/TR-253-2626, Golden, CO: Solar Energy Research Institute.

Murphy, L. M., forthcoming, "Moderate to Large Axisymmetric Deformation of Optical Membrane Surfaces," submitted for publication to the Journal of Solar Energy Engineering.

Murphy, L. M., and D. V. Sallis, June 1984, Analytical Modeling and Structural Response of a Stretched-Membrane Reflective Module, SERI/TR-253-2101, Golden, CO: Solar Energy Research Institute.

Roorda, T., 1965, "The Buckling Behavior of Imperfect Structural Systems," Journal of the Mechanics and Physics of Solids, Vol. 13, p. 267.

Roorda, J., Dec. 1967, "Some Thoughts on the Southwell Plot," Proc. Am. Soc. Civil Eng., Vol. 93, No. EM6.

Schaeffer, H. G., 1979, MSC/NASTRAN PRIMER - Static and Normal Mode Analysis: A Study of Computerized Technology, Mount Vernon, NH: Schaeffer Analysis, Inc.

Solkolnikoff, I. S., 1956, Mathematical Theory of Elasticity, 2nd edition, New York: McGraw-Hill.

Thompson, J. M. T., and G. W. Hunt, 1973, A General Theory of Elastic Stability, Chapter 2, New York: John Wiley and Sons. 
Thompson, J. M. T., and G. W. Hunt, 1983, Collapse: The Buckling of Structure in Theory and Practice, New York: Cambridge University Press.

Timoshenko, S. P., 1961, Theory of Elastic Stability, 2nd edition, New York: McGrawHill, pp. 313-318.

Timoshenko, S. P., and S. Woinowsky-Krieger, 1959, Theory of Plates and Shells, New York: McGraw-Hill, pp. 396-404.

Wood, R. L., 6 Jan. 1986a, "Completion of Test on a 3-Meter-Diameter Dual-Membrane Module," Internal Memorandum, Solar Energy Research Institute, Golden, CO.

Wood, R. L., Feb. 1986b, Single Stretched-Membrane, Structural Module Experiments, SERI/TR-253-2736, Golden, CO: Solar Energy Research Institute. 


\section{APPENDIX A}

\section{AN EIGENVALUE APPROACH TO DETERMINING THE CRITICAL BIFURCATION TENSION LOAD FOR A SINGLE-MEMBRANE MODULE DESIGN}

The equilibrium equations for the ring/membrane (single-membrane) combination used here predict the membrane tension at which a bifurcation of equilibrium for the assembly will occur. From Murphy and Sallis (1984) the appropriate ring frame equilibrium equations can be expressed as

$$
\frac{E I}{R}\left(\frac{V^{\prime \prime \prime}}{R}-\phi^{\prime \prime}\right)-\frac{G K}{R}\left(\phi^{\prime \prime}+\frac{V^{\prime \prime}}{R}\right)+\operatorname{RaT}_{0}\left(\frac{V^{\prime \prime}}{R}-\alpha\right)=0,
$$

for out-of-plane bending and

$$
\frac{\mathrm{GK}}{\mathrm{R}}\left(\phi^{\prime \prime}+\frac{\mathrm{v}^{\prime \prime}}{\mathrm{R}}\right)+\frac{\mathrm{EI}}{\mathrm{R}}\left(\frac{\mathrm{v}^{\prime \prime}}{\mathrm{R}}-\phi\right)-\mathrm{aT}_{\mathrm{o}} \rho(\phi-\alpha)=0,
$$

for frame twist. The prime (') represents differentiation with respect to the circumferential coordinate $\theta$, and EI, GK, and R represent the frame out-of-plane flexural rigidity, the torsional rigidity, and the mean radius, respectively. $a$ is the membrane radius, and $\rho$ is the distance of the membrane attachment from the frame cross-section torsional center (see Figure A-1). $T_{0}$ is the membrane operating tension, $v$

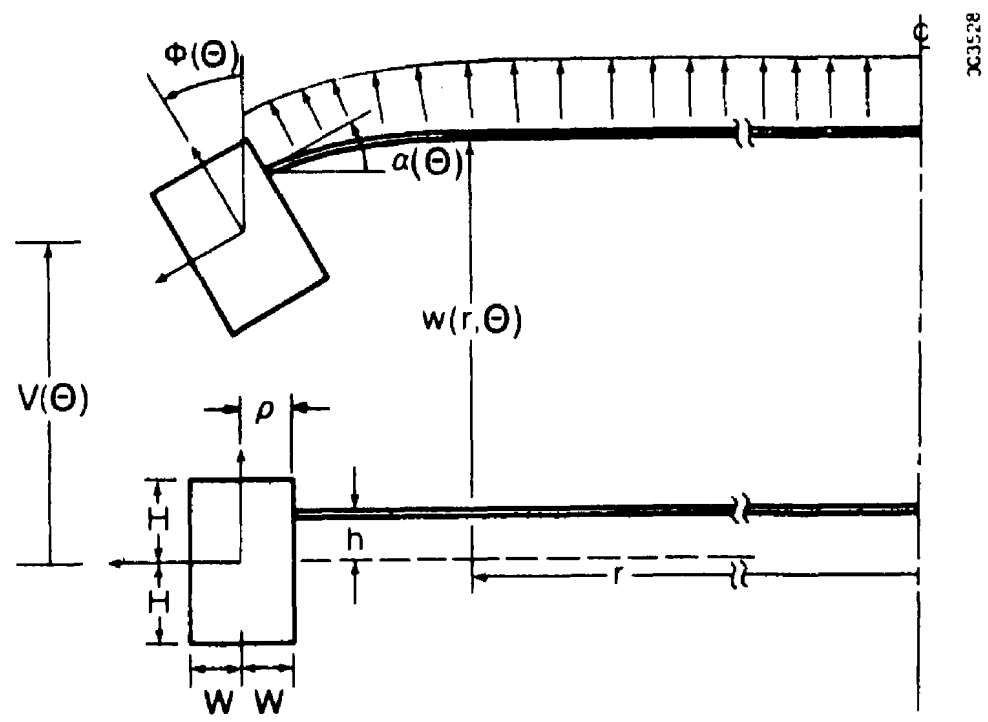

Figure A-1. Frame and Cross-Section Detail Showing Displacements and the Corresponding Directions and Applied Loading 
is the frame out-of-plane displacement increment,* $\phi$ is the frame rotation about its torsional center, and $\alpha$ is the angle the membrane makes with the horizontal at its point of attachment. $\alpha$ brings the coupling effect of the membrane into the frame Eqs. A-1 and $A-2$, and when it is set to zero Eqs. $A-1$ and A-2 degenerate to the classical ring-only equations.

If we now assume that in the buckled configuration the ring/membrane combination deforms in a harmonic displacement pattern, and that at the critical membrane tension the system is in a state of neutral equilibrium, then we can define displacement increments by

$$
v=c_{1} \cos (n \theta)
$$

and

$$
\phi=c_{2} \cos (n \theta) \text {, }
$$

where symmetry conditions determine the relative form of $\mathrm{v}$ and $\phi$ for the nth buckling mode. $C_{1}$ and $C_{2}$ are constant coefficients, and $n$ corresponds to the modal buckling shape.

The expression for a (the slope of the membrane at the frame attachment) can be determined from Eq. 17 in Murphy and Sallis (1984), which describes the shape of the membrane for the nonhomogeneous boundary condition. Thus, for a single, uniform harmonic frame displacement field defined by Eqs. A-3 and A-4, $\alpha$ is described by

$$
\alpha=\left.\frac{-d w}{d r}\right|_{r=a}=\frac{-d}{d r} \frac{1}{\pi}\left(\frac{r}{a}\right)^{n} \cos (n \theta) \int_{0}^{2 \pi}(v+\rho \phi) \cos n \psi d \psi,
$$

where $\mathrm{w}$ is the membrane deformation, normal to the surface, in the vertical direction, and $r$ is the radial coordinate. This equation reduces to

$$
\alpha=-\Delta\left(C_{1}+\rho C_{2}\right) \frac{n}{a} \cos (n \theta),
$$

where $\Delta$ is defined by

$$
\Delta=\left\{\begin{array}{l}
0, \text { if no membrane is present } \\
1, \text { if a membrane is present }
\end{array}\right.
$$

Substituting Eqs. A-3 through A-6 into Eqs. A-1 and A-2 will yield

$$
\left[\lambda n^{2}+1-T_{0} A \frac{a}{R}\left(1-\frac{\Delta}{n} \frac{R}{a}\right)\right] \frac{C_{1}}{R}+\left(\lambda+1+T_{0} A \frac{\rho}{R} \frac{\Delta}{n}\right) C_{2}=0,
$$

and

$$
\left[(\lambda+1) n^{2}+T_{0} B n \frac{R}{a} \Delta\right] \frac{C}{R}+\left[n^{2}+\lambda+T_{0} B\left(1+\frac{n}{a} \circ \Delta\right)\right] C_{2}=0,
$$

\footnotetext{
* Note that here, as in Murphy and Sallis (1984) and Murphy (1985), we are considering out-of-plane and rotation deformation increments relative to the initially flat compressed frame/membrane combination.
} 
where $\lambda, A$, and $B$ are defined by

$$
\begin{gathered}
A=\frac{R^{3}}{G K}, \\
B=\frac{R a \rho}{G K}=A\left(\frac{a}{R}\right)\left(\frac{\rho}{R}\right),
\end{gathered}
$$

and

$$
\lambda=\frac{\mathrm{EI}}{\mathrm{GK}} \text {. }
$$

Now the condition of neutral equilibrium at which a bifurcation of the equilibrium state can occur is given by the value of the tension ( $T_{0}$ ), which results in the determinant of the coefficients of $C_{1} / R$ and $C_{2}$ in Eqs. A-8 and A-9 being zero. Setting the determinant of the above two equations to zero results in a quadratic expression for $T_{0}=T(n) c ; R / M$ (i.e., the critical bifurcation tension for a ring with or without a membrane) given by $\mathrm{C} / \mathrm{M}$

$$
\begin{aligned}
& \left\{\left[(\lambda+1) n^{2}+T_{0} A \frac{\rho}{R} n \Delta\right]\left[(\lambda+1)+T_{0} A \frac{\rho}{R} \frac{\Delta}{n}\right]\right. \\
& \left.-\left[\lambda n^{2}+1-T_{0} A \frac{a}{R}\left(1-\frac{\Delta}{n} \frac{R}{a}\right)\right]\left[n^{2}+\lambda+T_{0} A \frac{a}{R} \frac{\rho}{R}\left(1+n \Delta \frac{\rho}{a}\right)\right]\right\}=0 .
\end{aligned}
$$

Equation $A-13$ can be cast in the following quadratic form:

$$
D T_{0}^{2}+E T_{0}-F=0 \text {, }
$$

where the critical bifurcation tension for a ring with or without a membrane $T(n, \rho){ }_{C ; R} / M$ is given by $T_{0}$, which can be expressed by

$$
T(n, 0)_{c ; R / M}=T_{0}=\frac{E}{2 D}\left[\left(1+\frac{4 F D}{E^{2}}\right)^{1 / 2}-1\right] \text {, }
$$

and where

$$
\begin{aligned}
& D=\left(\frac{R^{3}}{G K}\right)^{2}\left[\left(1-\frac{\Delta}{n} \frac{R}{a}\right)\left(\frac{a}{R}\right)^{2}\left(\frac{\rho}{R}\right)\left(1+\Delta n \frac{\rho}{a}\right)+\left(\frac{\rho}{R}\right)^{2} \Delta^{2}\right], \\
& E=\frac{R^{3}}{G K}\left[\left(n^{2}+\lambda\right) \frac{a}{R}\left(1-\frac{\Delta}{n} \frac{R}{a}\right)+(\lambda+1) 2 n \Delta \frac{\rho}{R}-\left(\lambda n^{2}+1\right) \frac{a}{R} \frac{\rho}{R}\left(1+n \frac{\rho}{a} \Delta\right)\right], \\
& E=\lambda\left(n^{4}-2 n^{2}+1\right) .
\end{aligned}
$$

The NASTRAN results discussed in the main body of the report were run with the tension load applied to the frame torsional center (i.e., $\rho=0$ ). We will discuss the corresponding case first since the resulting solution will approximate the more general solution that is valid as long as $\rho$ is small. For $\rho=0, E q . A-15$ will result in

$$
T_{0}=T(n, 0)_{c ; R / M}=T(n)_{c ; R / M}=\frac{E I}{R^{3}}\left[\frac{n^{4}-2 n^{2}+1}{\left(n^{2}+\lambda\right)\left(1-\frac{\Delta}{n}\right)}\right]=\frac{T(n)_{c ; R}}{\left(1-\frac{\Delta}{n}\right)},
$$

where $T(n)_{C ; R}$ is the critical compressive load on an identical frame loaded at its centroid to which no membrane is attached. $T(n)_{c ; R}$ as defined by Eq. A-17 can be written as 


$$
T(n)_{c ; R}=\frac{E I}{R^{3}}\left(\frac{n^{4}-2 n^{2}+1}{n^{2}+\lambda}\right) .
$$

Note that Eq. A-18 is identical to Timoshenko's (1961) corresponding (ring-only) solution given for the $n=2$ case.

For the $\rho=0$ case, the membrane provides a stiffening effect on the ring/membrane assembly that results in a critical buckling load, which is greater than the ring-only buckling load by a factor of 2.0 for the $n=2$ case and a factor of 1.5 for the $n=3$ case. Furthermore, for small values of $\rho / R$, the attachment offset causes an additional small stiffening effect that one can see by expanding Eqs. A-15 and A-16 in terms of $(\rho / R)$. Such an expansion when linearized in $(\rho / R)$ results in

$$
T(n, p)_{C ; R / M} \approx T(n)_{C ; R / M}\left(1-\frac{p}{R} \Omega\right) \text {, }
$$

where

$$
\Omega=\left[(\lambda+1) \Delta n-\left(n^{2}+\lambda\right)-\left(\lambda n^{2}+1\right)(1+\Delta n)\right] /\left[\left(n^{2}+\lambda\right)\left(1-\frac{\Delta}{n}\right)\right] .
$$

Comparative buckling ratios (i.e., corresponding to the buckling tension for the frame with a membrane, divided by the buckling tension for the ring/membrane combination when $\rho$ is zero) are presented in Table A-1 using the accurate form Eq. A-15 and the more limited approximation Eq. A-19, respectively. The effect of the offset is seen to be quite small, and the approximation (Eq. A-19) is quite adequate for the case considered.

\begin{tabular}{|c|c|c|}
\hline $\mathrm{n}$ & 2 & 3 \\
\hline$T(n)_{C ; R}(N / m)$ & 14,720 & 66,353 \\
\hline $\begin{array}{l}T(n){ }_{c} ; R / M^{(N / m)} \\
{[T(n, \rho)} \\
c ; R / M^{] /[T(n}\end{array}$ & 29,439 & 99,529 \\
\hline
\end{tabular}

Table A-1. Critical Membrane Tension Loads for Several Geometric Configurations ${ }^{\mathrm{a}}$

Frame/membrane combination

Equations $A-15$ and $A-17$

Equations $A-19$ and $A-17$

Ring-only case, $\Delta=0$

Equations $A-15$ and $A-17, \Delta=0$

1.0130

Equations $A-19$ and $A-17, \Delta=0$

1.0130

1.0118

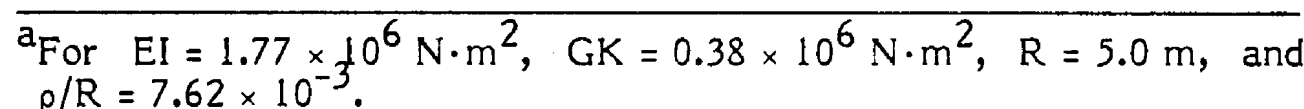




\section{APPENDIX B \\ A NONLINEAR AXISYMMETRIC MEMBRANE/SUPPORT FRAME LOAD DEFORMATION RELATIONSHIP}

We can use an approximate but simple geometric/strength-of-materials approach to determine a nonlinear load deformation relationship for a uniformly loaded prestretched circular membrane with a compliant support frame. The current approximate analysis applies to a single-membrane frame/membrane combination. Uniform strain in the membrane is assumed for axisymmetric deformation. The result, which predicts lateral deformation response for the membrane, is in reasonably good agreement with classical nonlinear elasticity solutions (see Murphy, forthcoming) and can be used for design and system trade-off studies, as well as to predict the overall effect on membrane tension. Results also show that a relatively large amount of axisymmetric deformation is required before the uniform tension state of the membrane is significantly altered. The model predicts a differential pressure, center deformation relationship given by

$$
\Delta P=\frac{4 \mathrm{~T}_{\mathrm{o}}}{\mathrm{a}}\left(\frac{\mathrm{w}_{\mathrm{o}}}{\mathrm{a}}\right)+\frac{8}{3}\left(\frac{\mathrm{K}_{\mathrm{m}}}{1+\mathrm{K}_{\mathrm{m}} / \mathrm{K}_{\mathrm{R}}}\right)\left(\frac{\mathrm{w}_{\mathrm{o}}}{\mathrm{a}}\right)^{3},
$$

and an incremental tension $\Delta \mathrm{T}_{0}$, deformation relationship given by

where

$$
\Delta \mathrm{T}_{\mathrm{o}}=\frac{\mathrm{a}\left(\mathrm{K}_{\mathrm{m}}\right) \frac{2}{3}\left(\frac{\mathrm{w}_{\mathrm{O}}}{\mathrm{a}}\right)^{2}}{\left(1+\mathrm{K}_{\mathrm{m}} / \mathrm{K}_{\mathrm{R}}\right)},
$$

$$
\begin{aligned}
\Delta \mathrm{P} & =\text { uniform pressure increment loading } \\
\mathrm{a} & =\text { radius of membrane } \\
\mathrm{w}_{\mathrm{O}} & =\text { center deflection of membrane } \\
\mathrm{K}_{\mathrm{m}} & =\text { radial stiffness of membrane } \\
\mathrm{K}_{\mathrm{R}} & =\text { radial stiffness of support ring } \\
\mathrm{T}_{\mathrm{O}} & =\text { pretension in the membrane } \\
\Delta \mathrm{T}_{\mathrm{O}} & =\text { increase in membrane tension caused by the application of } \Delta \mathrm{P} .
\end{aligned}
$$

We can interpret the terms in Eq. B-l in the following manner. The first term corresponds to the membrane lateral stiffness $\left(4 \mathrm{~T} / \mathrm{a}^{2}\right)$ times the deflection $\mathrm{w}_{0}$. The second term is the so-called nonlinear diaphragm stiffness

$$
\left[\frac{8}{3 a}\left(\frac{K_{m}}{1+K_{m} / K_{R}}\right)\left(\frac{w_{0}}{a}\right)^{2}\right],
$$

times the membrane center deflection $w_{0}$. This second stiffness term arises from the in-plane strains induced by the out-of-plane deformation. The two stiffnesses act in parallel; the lateral membrane stiffness results from a change in the membrane's shape that is not accompanied by a change in strain in this approximation (only a change in shape), and the diaphragm stiffness results from stretching of the membrane/frame combination in its plane, which in turn results from large lateral deformations. The membrane stiffness dominates until the distortion becomes quite large, at which point the diaphragm stiffness becomes significant, adding appreciable rigidity to the system. 
The second term for the diaphragm stiffness agrees with the classical solution for an initially untensioned membrane as developed by Timoshenko (1961) for a rigid frame (i.e., when $\left.\mathrm{K}_{\mathrm{R}}+\infty\right)$. This term is also easily derived using the principle of minimum potential energy assuming a uniform increment in the strain state over the entire membrane as in Roorda (1965). When arriving at the expression for the diaphragm stiffness, we assumed that a displacement function for the membrane had the form of a shallow spherical surface. Its form in terms of the radial coordinate $r$, the membrane radius $a$, and the center deflection $w_{0}$ is

$$
w(r)=w_{0}\left[1-\left(\frac{r}{a}\right)^{2}\right] .
$$

The in-plane membrane stiffness $\mathrm{K}_{\mathrm{m}}$ is given by

$$
\mathrm{K}_{\mathrm{m}}=\frac{E t}{\mathrm{a}(1-v)} \text {, }
$$

where $E$ is Young's modulus, $t$ is the membrane thickness, and $v$ is Poisson's ratio, respectively, corresponding to the membrane.

The ring stiffness enters into the expression for the diaphragm stiffness because it acts in parallel with the diaphragm response. The ring stiffness can be expressed by

$$
\begin{aligned}
K_{R} & =\frac{E_{R} A_{R}}{R a} \\
K_{R} & =\frac{\frac{E_{R} A_{R}}{R a}}{I+\frac{A_{R}}{I_{y}} h^{2}}
\end{aligned}
$$

if the ring is loaded through its center of torsion

if the ring is loaded

eccentrically (see Figure B-1),

where $E_{R}$ is Young's modulus, $A_{R}$ is the cross-section area, $R$ is the mean radius $(\geq a), I_{y}$ is the out-of-plane bending moment of inertia, $H$ is the farthest distance of the crost section to the neutral axis of the $r i n g$, and $h$ is the distance from the ring neutral axis to the membrane attachment (see Figure B-1), respectively, all corresponding to the ring support frame.

We can approximate the membrane tension increment $\Delta T_{0}$, which is determined by evaluating the strain increment (assumed to be uniform over the surface) in the membrane corresponding to only the diaphragm deformation. The resulting strain increment $\Delta \varepsilon$ is given by

$$
\Delta \varepsilon \approx \frac{1}{a} \int_{0}^{a-\delta}\left(1+\frac{1}{2} w_{r}^{2}\right) d r-1 \approx-\frac{\delta}{a}+\frac{2}{3}\left(\frac{w_{0}}{a}\right)^{2},
$$

where $\delta$ is the edge deflection of the membrane, which can be related to the strain through force equilibrium by

$$
-\frac{\delta}{\mathrm{a}}=\frac{\mathrm{K}_{\mathrm{m}}}{\mathrm{K}_{\mathrm{R}}} \Delta \varepsilon .
$$

* Though the current analysis is derived for a single-membrane structure, the fundamental difference for a double-membrane structure will be in the additional frame stiffness caused by the coupling with the second membrane. 


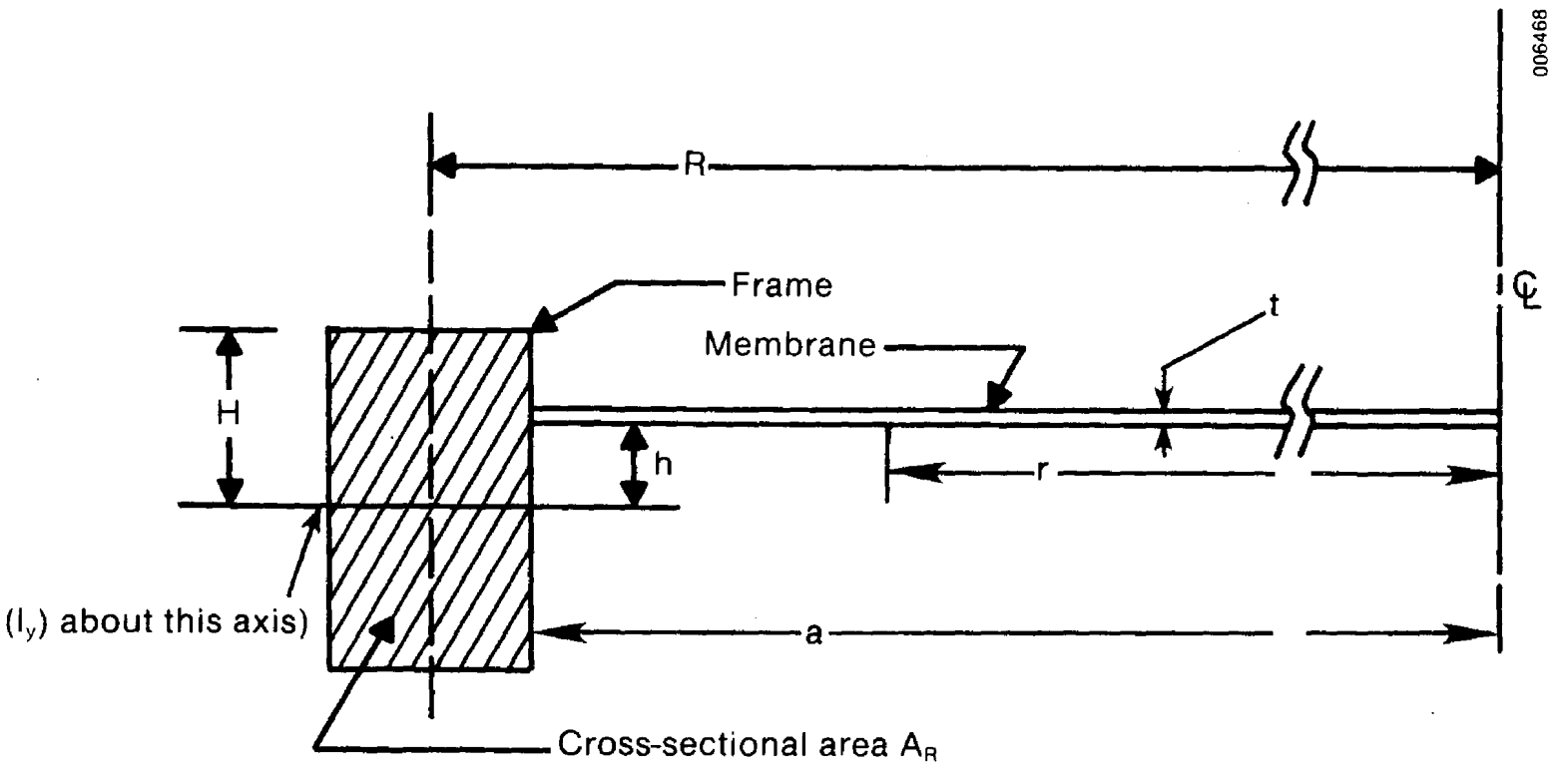

Figure B-1. Frame Membrane Geometry

Then solving Eqs. B-6 and B-7 for $\Delta \varepsilon$ and eliminating $\frac{\delta}{a}$ results in

$$
\Delta \varepsilon=\frac{\frac{2}{3}\left(\frac{\mathrm{w}_{\mathrm{O}}}{\mathrm{a}}\right)^{2}}{1+\mathrm{K}_{\mathrm{m}} / \mathrm{K}_{\mathrm{R}}} .
$$

Figure B-2 illustrates the response of the ring/frame combination described by Eqs. B-1 and B-2. It is seen that for high tension, low pressures, low frame stiffness, or low membrane stiffness the diaphragm stretching of the membrane/frame combination has little effect, and the load deformation response is nearly linear. This occurs because high initial tensions will dominate small tension increments caused by in-plane stretching of the membrane/frame combination. Further, for small membrane-stiffness and low frame-stiffness conditions, rather large out-of-plane deformations are required before any appreciable in-plane strains and tension increments can occur. Thus, it is seen that the load deformation relationship can be nearly linear for a fairly wide range of parameters. 


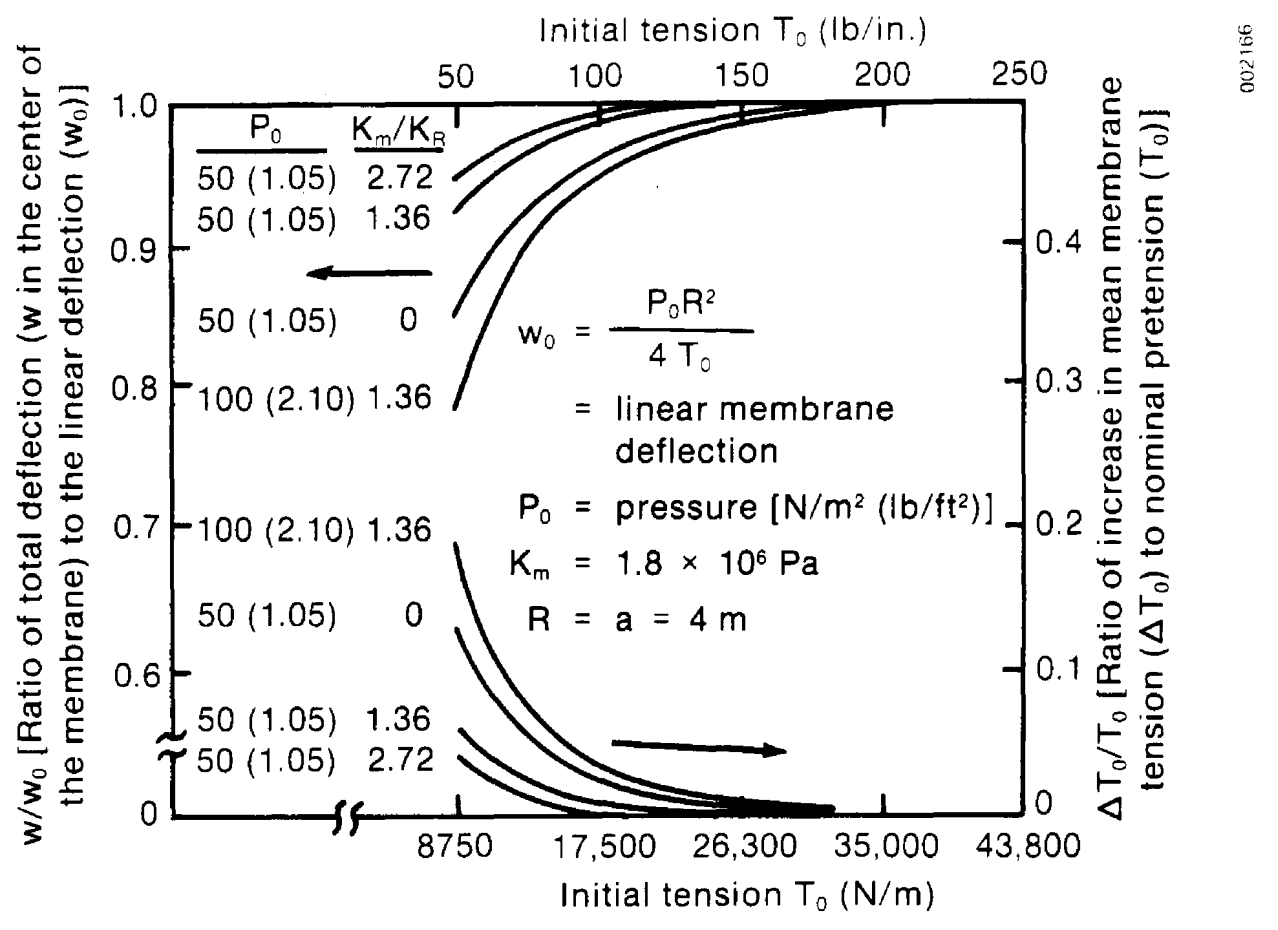

Figure B-2. Membrane Center Deformation Ratio and Fractional Tension Increase as a Function of Initial Membrane Tension for Several Pressure Loadings and Support Ring Stiffnesses [The deformation ratio corresponds to the total nonlinear membrane deformation divided by linear membrane deformation (both measured in the center of the membrane). A pressure loading of $50 \mathrm{~N} / \mathrm{m}^{2}$ corresponds to the approximate maximum loading from a $9-\mathrm{m} / \mathrm{s}$ wind.] 


\section{APPENDIX C}

\section{IN-PLANE BUCKLING OF RING/MEMBRANE COMBINATION}

The critical bifurcation tension of a ring frame onto which a single initially tensioned membrane is mounted can be modeled to a first approximation as a compressed ring on an elastic foundation. Meck (1969) defined the governing buckling equation for a ring subjected to a varying radial load $[p(\theta)]$, but with a mean compressive force $N$, in terms of the radial displacement $(u)$ and ring radius $(R)$ :

$$
\frac{E I}{R^{2}}\left(u^{\prime \prime \prime}+2 u^{\prime \prime}+u\right)^{\prime}+N\left(u^{\prime \prime}+u\right)^{\prime}+N^{\prime}\left(u^{\prime \prime}+u\right)-p^{\prime} R^{2}=0, \quad(C-1)
$$

where as before the (') superscript denotes differentiation with respect to $\theta . E$ and $I_{X}$ are the frame modulus and the in-plane bending moment of inertia, respectively. If the membrane stiffness can be expressed as $\mathrm{K}_{n}$, analogous to an elastic foundation modulus for a given modal shape, then

$$
\frac{E I}{R^{2}}\left(u^{\prime \prime \prime}+2 u^{\prime \prime}+\dot{u}\right)^{\prime}+N\left(u^{\prime \prime}+u\right)^{\prime}+N^{\prime}\left(u^{\prime \prime}+u\right)+K_{n} u^{\prime} R^{2}=0 \text {. }
$$

In addition, if the displacements are small with respect to the radius, then $\mathrm{N}^{\prime}$ will be small compared to $\mathrm{N}$ and the third term can be omitted (Timoshenko and WoinowskyKrieger 1959). The appropriate buckling condition can be written

$$
\frac{E I_{x}}{R^{2}}\left(u^{\prime \prime \prime}+2 u^{\prime \prime}+u\right)^{\prime}+N\left(u^{\prime \prime}+u\right)^{\prime}+K_{n} u^{\prime} R^{2}=0 .
$$

Letting the compressive force $\mathrm{N}$ be denoted by $\left(T_{0} R\right)$, the critical tension load for an assumed modal deformation of the form

$$
u=a \cos n \theta
$$

can be described by

$$
\left(T_{0}\right)_{c r ; i n-p l a n e}=\frac{E I_{x}}{R^{3}}\left(n^{2}-1\right)+\frac{R_{n}}{n^{2}-1} \text {. }
$$

The first term of Eq. C-5 corresponds to the buckling load for the ring only, and the second term results from the stiffening effect of the foundation (or the membrane/ attachment assembly). $K_{n}$ is the radial stiffness of the membrane/attachment combination, which is composed of the membrane radial stiffness $K_{m}(n)$ acting in series with the attachment radial stiffness $K_{a t}$. Hence $K_{n}$ can be written

$$
K_{n}=\frac{K_{a t} K_{m}(n)}{K_{a t}+K_{m}(n)}
$$


where $K_{m}(n)$ denotes the membrane stiffness corresponding to the $n$th radial mode of deformation.

Two limiting cases for $\mathrm{K}_{\mathrm{n}}$, corresponding to a rigid attachment of the membrane to the frame and a low stiffness attachment, are particularly interesting and result in

$$
K_{n}=\left\{\begin{array}{ll}
K_{m}, & \text { for } K_{a t}+\infty \\
k_{a t}, & \text { for } k_{m}+\infty
\end{array},\right.
$$

respectively.

To evaluate Eqs. C-5 and C-6, we must determine $K_{m}(n)$ as a function of modal deformation number $\mathrm{n}$ by considering the plane stress problem for the membrane and determining the stiffness that corresponds to the displacement of the membrane edge

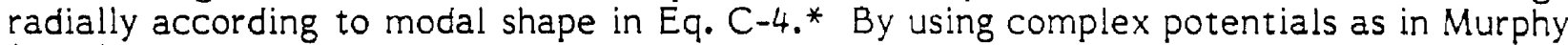
(1985), the solution is obtained in a straightforward, albeit somewhat tedious, manner (see Solkolnikoff 1956). The appropriate stiffness for a radial constraint is

$$
K_{m}(n)=\frac{E_{m}^{t} m}{a} \frac{n^{2}-1}{2 n-1+v} \text {. }
$$

Here $E_{m}, t_{m}, v$, and a represent the modulus, thickness, Poisson ratio, and radius, respectively, all corresponding to the membrane.

Eq. C-5 shows that for reasonably high values of $\mathrm{K}_{\mathrm{m}}$, the minimum value of $\mathrm{T}_{\mathrm{o}}$ will not occur for $n=2$. For example, the ring/membrane combination considered in the discussion of Figure 3-3 results in the critical in-plane buckling loads given in Table C-1 that corresponds to various levels of attachment stiffness, where Eqs. C-6 and C-7 are used by Eq. C-8.

To compare, note that the fundamental out-of-plane buckling mode is characterized by $\mathrm{n}=2$ and a critical tension of $\left(T_{0}\right)_{\mathrm{cr}}$; in-plane $=29,8000 \mathrm{~N} / \mathrm{m}$.

* We assumed that for each $n$, the corresponding displacement field can be linearly superimposed upon the uniform prebuckled deformation. Hence, we are not concerned with out-of-plane membrane deformations or buckling here. 
Table C-1. Critical In-Plane Buckling Parameters for the Ring/Membrane Combination Shown in Figure 2-19

\begin{tabular}{llll}
\hline $\begin{array}{l}\text { Attachment } \\
\text { Stiffness } \\
\mathrm{K}_{\text {at }}(\mathrm{Pa})\end{array}$ & $\left(\mathrm{T}_{\mathrm{o}}\right)_{\mathrm{Cr}} ;$ in-plane & & \\
\hline $1 \mathrm{~N} / \mathrm{m})$ & $\mathrm{n}_{\text {crit }}$ & $\mathrm{K}_{\mathrm{m}}(\mathrm{Pa})$ \\
$1.0 \times 10^{35}$ & $2.45 \times 10^{6}$ & 17 & $9.09 \times 10^{7}$ \\
$1.0 \times 10^{9}$ & $2.32 \times 10^{6}$ & 16 & $8.57 \times 10^{7}$ \\
$1.0 \times 10^{7}$ & $7.21 \times 10^{5}$ & 11 & $5.92 \times 10^{7}$ \\
$1.0 \times 10^{5}$ & $7.87 \times 10^{4}$ & 4 & $2.16 \times 10^{7}$ \\
\hline
\end{tabular}

Notes

$$
\begin{aligned}
\mathrm{t}_{\mathrm{m}} & =0.254 \mathrm{~mm} \\
\mathrm{E} & =E_{\mathrm{m}}=207 \mathrm{G} \mathrm{Pa} \\
v_{\mathrm{m}} & =0.3 \\
\mathrm{R} & =5.0 \mathrm{~m} \\
\mathrm{I}_{\mathrm{x}} & =1.86 \times 10^{-6} \mathrm{~m}^{4} \\
\mathrm{~K}_{\mathrm{m}}(\mathrm{n}) & =1.05 \times 10^{7}\left(\frac{\mathrm{n}^{2}-1}{2 \mathrm{n}-0.7}\right)
\end{aligned}
$$




\section{APPENDIX D}

\section{AMPLIFICATION FACTORS FOR INITIAL IMPERFECTIONS AND LATERAL LOADING CORRESPONDING TO SINGLE-MEMBRANE DESIGNS}

\section{D.1 Amplification of Initial Imperfections}

The stability analysis presented in Section 3.0 of this report provides a context from which we can describe the amplification of initial imperfections that are present in the frame before attaching the tensioned membrane to it. Once we can define the critical tension level corresponding to a bifurcation of equilibrium, we can use the Southwell approach to estimate the amplification of the initial imperfection, whether or not it is generated by an initial out-of-plane displacement in the frame or an out-of-plane external load applied to the frame. We can use the Southwell approach to predict what that amplified displacement is as a function of the ratio of the membrane tension load to the critical bifurcation tension load. Using the Southwell approach, the amplification of the initial $\left[A_{0}(n)\right]$ imperfection, corresponding to the nth mode, is described by the following equation (McGuiness 1961):

$$
\frac{A_{i}(n)}{A_{0}(n)}=\frac{1.0}{1-T_{0} / T(n)_{C} ; R / M},
$$

where $A_{i}(n)$ is the amplified deformation corresponding to the initial frame imperfection $A_{o}(n)$ of the $n$th mode, $T_{O}$ is the working tension in the membrane, and $T(n) c ; R / M$ is the critical membrane tension corresponding to a bifurcation of equilibrlum of the frame/membrane assembly as defined earlier in Eq. A-17 corresponding to the nth mode. Note that Eq. D-1 is valid for a case in which the out-of-plane imperfection is defined by a single mode of deformation.

The accuracy of the approach will be acceptable only up to a point because the amplification Eq. D-1 based on the Southwell procedure is singular at the critical system bifurcation tension (for the corresponding mode), and it becomes increasingly less accurate as the singularity is approached. However, if the membrane tension load is sufficiently below the critical level of interest, then reasonable accuracy should occur as can be inferred from Figure 3-3. In fact, we will show that the approach is useful for tension levels up to about $60 \%-70 \%$ of the critical bifurcation load for the frame/membrane assembly, at least for the case illustrating the $n=2$ mode. Thus, a plot of the predicted value of the amplification factor $\left[A_{i}(n) / A_{o}(n)\right]$ versus the ratio of operating tension $\left(T_{0}\right)$ to critical tension $[T(n) c ; R / M]$ using either the NASTRAN results or the Southwell approach (Eq. D-1), demonstrates good correlation (see Figure D-1). We anticipate that the tension level at which the two curves start to diverge will decrease as the initial imperfections increase.

Imperfections in general cannot be described by a single mode shape but rather are most accurately described by a linear superposition of a number of harmonics, although certain modes will certainly dominate. The Southwell approach can still apply to a system experiencing a more general deformation pattern as described in the work of Ariaratnam (1961) and Roorda (1967). This study takes a linear modal approach in which the total imperfection field $w_{o}(\theta)$ is described by a series of modal contributions $f_{n}(\theta)$ given by the following expression:

$$
w_{0}(\theta)=\sum_{1}^{\infty} A_{0}(n) f_{n}(\theta)
$$




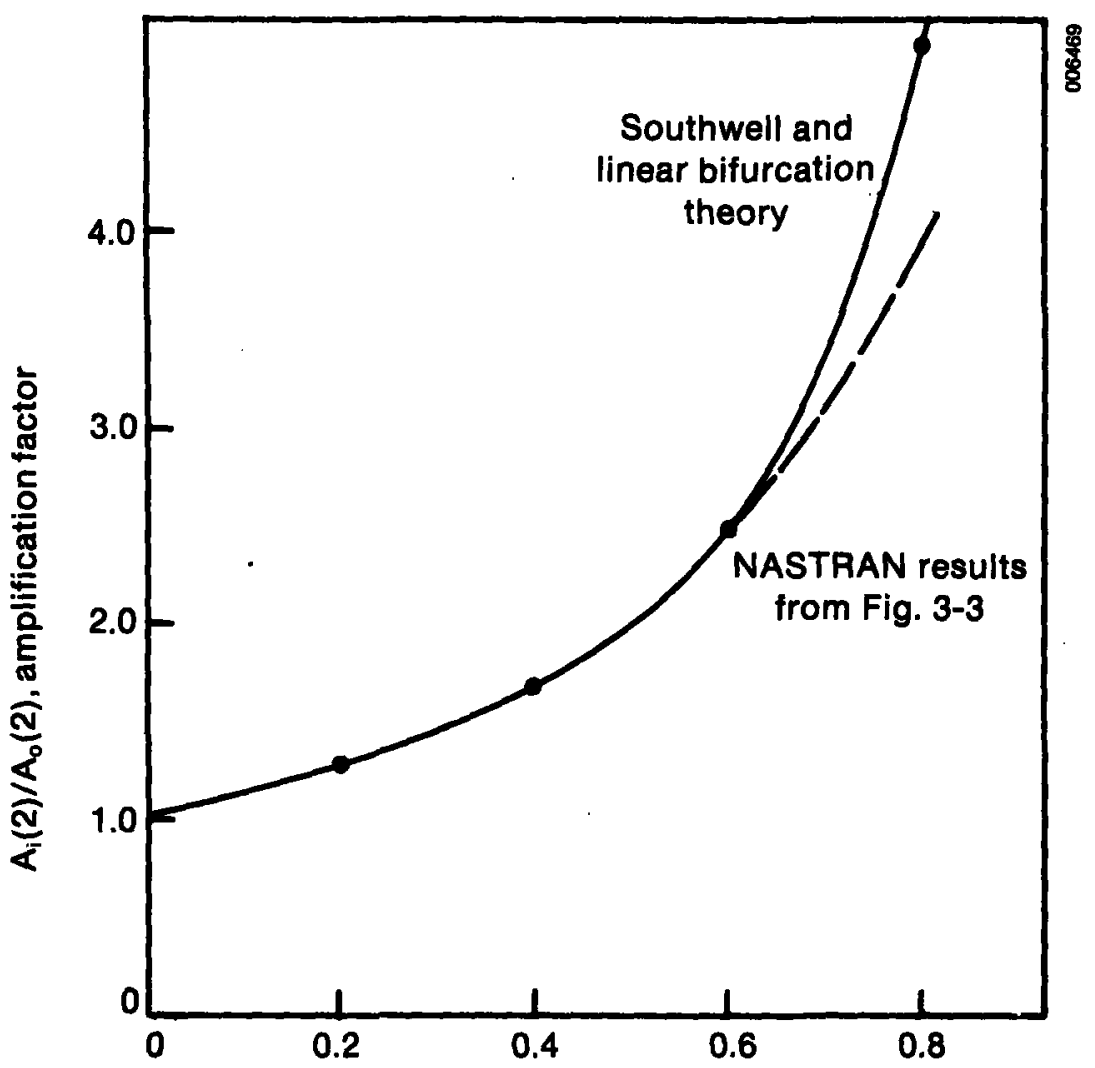

$T_{0} / T_{C A}$, ratio of membrane tension to critical membrane tension

Figure D-1. Ring Lateral Deformation Amplification Factor versus Operating/Critical Tension Ratio $(\mathbf{n}=\mathbf{2})$

where $f_{n}(\theta)$ is the displacement function, which is assumed to be orthogonal to all other $f_{j}(\theta)$ for $j \neq n$. Then the total displacement $w(\theta)$ at some load can be described by

$$
w(\theta)=\sum_{1}^{\infty} \frac{A_{0}(n)}{1-T_{0} / T(n)_{c} ; R / M} f_{n}(\theta) .
$$

Certain terms of $w(\theta)$ will dominate since not only will certain modes of the initial imperfection be predominant but the critical tensioning ratio will have a filtering effect on mode shapes where $T(n)_{c ; R / M}$ greatly exceeds $T_{0}$; and an amplification effect on the modes for which $T(n)_{c ; R / M}$ is close to $T_{0}$ will occur. An illustration of this effect follows. 
Table D-1 illustrates the implication of the Southwell predictions on the design of a ring/membrane combination when considering initial imperfections. This table corresponds to a ring/membrane similar to the one discussed previously that is somewhat more rigid with an increased critical bifurcation load. The ring frame has initial imperfections, characterized by two simultaneous out-of-plane mode shapes corresponding to $n=2$ and $n=3$, respectively. The $5-m$ radius frame operates under a nominal membrane tension of $17,500 \mathrm{~N} / \mathrm{m}$ and has a critical bifurcation load for the frame/membrane combination of $35,000 \mathrm{~N} / \mathrm{m}$ in the $\mathrm{n}=2$ mode and $112,000 \mathrm{~N} / \mathrm{m}$ in the $\mathrm{n}=3$ mode. The maximum initial vertical displacement of the frame for the $\mathrm{n}=2$ mode and the $n=3$ mode are 4 and $2 \mathrm{~mm}$, respectively. At one-half the critical load for the $\mathrm{n}=2$ case, the maximum out-of-plane deformation for the $\mathrm{n}=2$ case would double whereas it would only increase by $19 \%$ for the $n=3$ case because the $n=3$ mode results in a much higher critical bifurcation load than occurs for the $n=2$ mode. Hence, the operating tension is a much smaller percentage of the critical bifurcation level for the $\mathrm{n}=3$ mode than it is for the $\mathrm{n}=2$ mode. The rms surface errors corresponding to the nonaxisymmetric frame and membrane deformation implied with the modal shape and denoted as $\beta_{2}$ (Murphy and Sallis 1984)* are also given. As could be anticipated from the results in Murphy and Sallis, since the displacement doubles, the rms surface error average also doubles. However, for the $n=3$ case, the maximum displacement increase was $19 \%$ and therefore the rms surface error increase will be only $19 \%$.

The implication of the results in Table D- 1 is that the frame should be designed so that the nominal operating load (tension) in the membrane will be less than some factor below the critical bifurcation load for the $n=2$ mode, since the $n=2$ mode is the lowest fundamental buckling load of the ring. Clearly the actual operating tension should be a

Table D-1. Amplification of Mode Shapes

\begin{tabular}{lrr}
\hline $\mathrm{R}=5.0 \mathrm{~m}$ & $\mathrm{n}=2$ & $\mathrm{n}=3$ \\
\hline $\mathrm{T}_{\mathrm{O}}(\mathrm{N} / \mathrm{m})$ & $17,500.00$ & $17,500.00$ \\
$\mathrm{~T}(\mathrm{n}) \mathrm{C} ; \mathrm{R} / \mathrm{M}(\mathrm{N} / \mathrm{m})^{\mathrm{a}}$ & $35,000.00$ & $112,000.00$ \\
$\mathrm{v}_{\max }(\mathrm{initial})(\mathrm{mm})$ & 4.00 & 2.00 \\
$\mathrm{v}_{\max }(\mathrm{mm})$ & 8.00 & 2.38 \\
$\beta_{2}($ initial $)(\mathrm{mrad})$ & 1.13 & 0.69 \\
$\beta_{2}(\mathrm{mrad})$ & 2.26 & 0.82 \\
${ }^{\mathrm{a}}$ The frame was assumed to have an operating-to- \\
critical tension ratio of $\mathrm{T}_{\mathrm{O}} / \mathrm{T}(\mathrm{n}) \mathrm{C}_{\mathrm{C} ; \mathrm{R} / \mathrm{M}}=1 / 2$.
\end{tabular}

* The rms surface error $\beta_{2}$ is calculated by considering the area averaged square of the slope error as measured from the desired configuration. For the purposes of discussion here we can consider the desired configuration as flat. The initial imperfection in the frame introduces an incremental "scalloped" surface contour since the edge of the membrane must follow the contour displacement relative to the flat configuration of the deformed frame. 
function of the accuracy attainable in the frame itself before adding the membrane. If this cannot be determined a priori, so that a surface error can be determined from it and the tension levels adjusted accordingly (so that acceptable error levels result), then we recommend that in general the design should not go above roughly $25 \%$ of the critical tension corresponding to a bifurcation of equilibrium for the membrane/frame assembly. At $25 \%$ of the critical bifurcation load, the tension in the membrane would only magnify the initial imperfections by roughly $33 \%$. In any case, it would seem prudent to always design the frame so that the operating tension is no more than $50 \%$ of the critical bifurcation load (at $n=2$ ) since (ignoring for the moment the optical imperfections) this is a rather standard structural design approach, and because at loads above this level, the $n=2$ initial imperfections will more than double.

\section{D.2 Approximate Amplification Factors for Lateral Loads}

Designers can also use the Southwell approach in certain cases to estimate the lateral frame deformations corresponding to pressure loading within certain limitations.* Consider the pressure loading normal to the frame, which in turn is supported periodically at three equispaced, circumferentially placed vertical supports as defined in Murphy and Sallis (1984). Then, the Southwell approach can be used in conjunction with an analysis that considers only the lateral loading and deformation of the frame such as in Timoshenko (1961) to determine a correction (amplification) factor accounting for the frame compression. Since the total lateral pressure load on the membrane must be transmitted to the frame, the load resultant of the frame can be approximated by an equivalent uniform line load. The line load can then be viewed in turn as an equivalent frame imperfection that the tensioned frame will amplify. To illustrate this approach, consider Figure D-2, which plots the peak lateral frame deformation as a function of tension ratio in the membrane for different pressure levels. The tension ratio is defined as the operating tension in the membrane divided by the critical bifurcation tension of the membrane/frame combination for the particular mode under consideration. In Figure $\mathrm{D}-2$, the mode corresponds to $\mathrm{n}=3$ because the frame is supported in its operating condition at three equidistant points as discussed at length in Murphy and Sallis (1984). Note that $T(3)$ C:R/M $(=99,529 \mathrm{~N} / \mathrm{m})$ is the critical load corresponding to $n=3$, for the frame with radius $R=5.0 \mathrm{~m}$, a flexural rigidity of $E I=1.77 \times 10^{6} \mathrm{~N} \cdot \mathrm{m}^{2}$, and a torsional rigidity of $G K=0.38 \times 10^{6} \mathrm{~N} \cdot \mathrm{m}^{2}$.

The initial lateral displacement corresponds to the no-tension lateral displacement as given by McGuiness ( 1961 ), which is subjected to a uniform live load distribution of magnitude $\mathrm{P}_{\mathrm{L}}\left(=\rho \mathrm{P}_{\mathrm{o}} \mathrm{R}^{2}\right)$ applied laterally to the plane of the ring and distributed around the circumference of a ring that is supported at three equidistant points. The resulting expression (McGuiness 1961) for the $n=3$ case is given in terms of tabulated parameters $\lambda_{1}$ and $\lambda_{2}$ by

$$
\begin{aligned}
\left.v_{\max }\left(T_{0}=0\right)\right|_{n}=3 \frac{P_{L} R^{3}}{n^{4}}\left(\frac{\lambda_{1}}{4 E I}+\frac{\lambda_{2}}{4 G K}\right) & =\frac{\pi P_{0} 5^{5}}{(4) 3^{4}}\left(\frac{3.261}{E I}+\frac{0.35892}{G K}\right) \\
& =8.445 \times 10^{-5} P_{O},
\end{aligned}
$$

* The limitations relate to how closely the tension approaches the critical bifurcation level. 


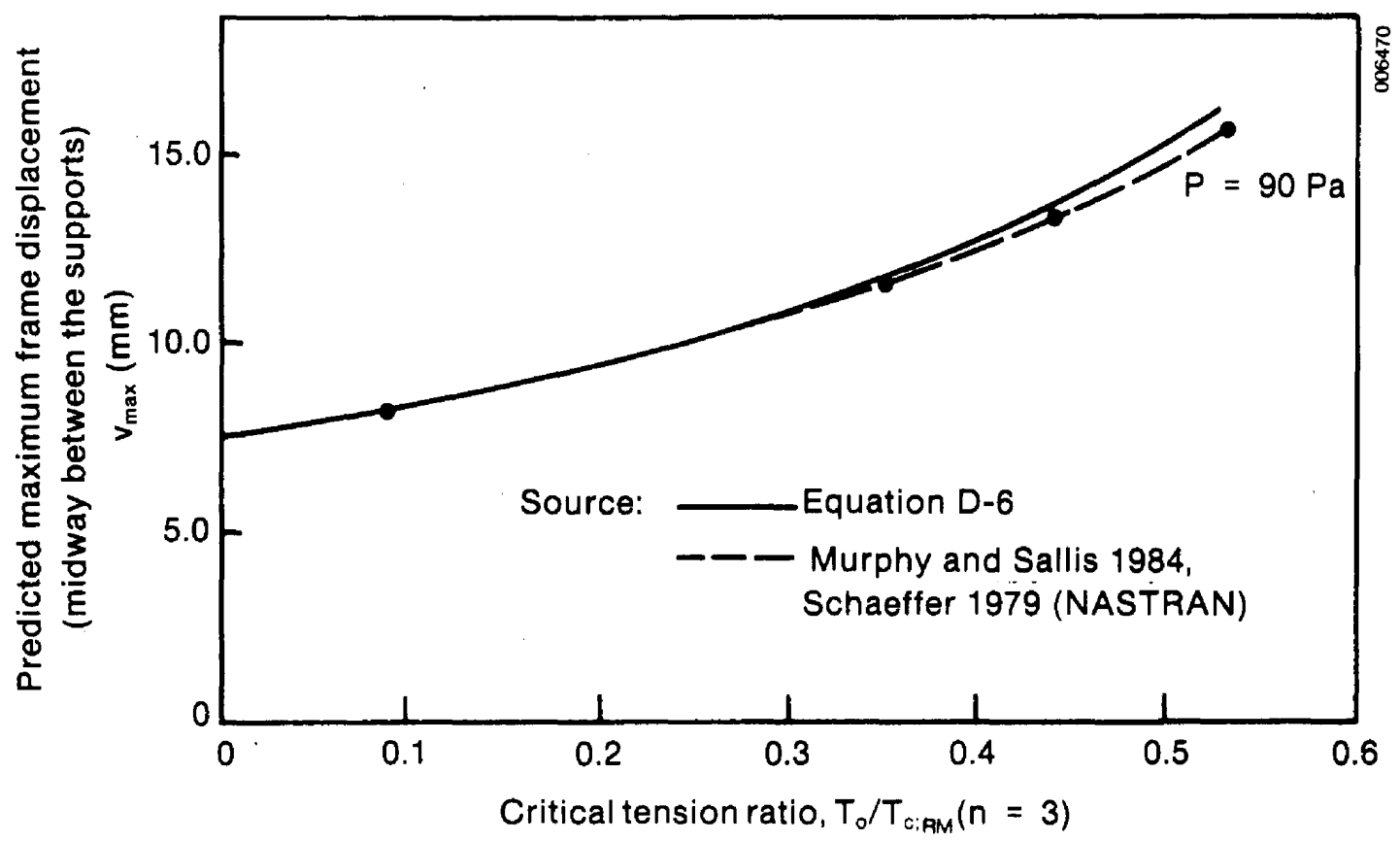

Figure D-2. Predicted Maximum Frame Displacement Midway between the Supports as a Function of Tension Ratio for Two Lateral Pressure Loading Conditions

where $P_{L}$ is related to the applied lateral pressure $P_{0}$ by

$$
P_{L}=\pi P_{O} R^{2} \text {. }
$$

Then the total displacement, accounting for the tension $T_{0}$, is found by combining Eqs. D-1 and D-4 to give

$$
v_{\max }\left(T_{0}\right)=\frac{v_{\max }\left(T_{0}=0\right)}{1-T_{O} / T_{C} ; R / M}=\frac{8.445 \times 10^{-5} P_{0}}{1-T_{0} / 99,529} \text { for } n=3 \text {, }
$$

where $T_{C ; R} / M^{(3)}(=99,529 \mathrm{~N} / \mathrm{m})$ is calculated from Eqs. $A-17$ and $A-18$ for the corresponding ring.

Although the assumption of uniform net load on the ring in the simple approach is not valid (the uniform load assumed is equal to the average of the true load that can have minimum-to-peak variations of more than a factor of 2 as seen in Murphy and Sallis [1984]), quite good agreement exists between Eq. D-4 and both the NASTRAN and the analytical solutions. Thus for the case studied, the lateral deformation of the frame is not sensitive to the distribution of the lateral load induced by the membrane. 


\section{SELECTED DISTRIBUTION LIST}

Acurex Solar Corporation

485 Clyde Ave.

Mt. View, CA 94042

Mr. Don Duffy

Advanco Corporation 40701 Monterey Ave.

Palm Desert, CA 92260

Mr. Byron Washom

Arizona Public Service Company

P.O. Box 21666

Phoenix, AZ 85036

Mr. Eric Weber

Battelle Pacific NW Laboratory

P.O. Box 999

Richland, WA 99352

Dr. Ben Johnson

Mr. Tom A. Williams

Bechtel Corporation

P.O. Box 3965

San Francisco, CA 94119

Mr. Pascal DeLaquil

Black and Veatch Consulting Engineers

1500 Meadow Lake Parkway

Kansas City, MO 64114

Dr. Charles Grosskreutz

Brumleve, Mr. Tom

Consultant

1512 N. Gate Road

Walnut Creek, CA 94598

Dan-Ka Products, Inc.

3862 South Kalamath

Englewood, CO 80110

Mr. Daniel Sallis

Department of Civil Engineering

Colorado State University

Ft. Collins, CO 80523

Mr. John Peterka, Ph.D.

Professor, Civil Engineering
Department of Civil Engineering

University of Nebraska

60 th \& Dodge Street

Omaha, NE 68182-0178

Mr. Chris Tuan, Ph.D.

Department of Energy/ALO

P.O. Box 1500

Albuquerque, NM 87115

Mr. Dean Graves

Mr. Nyles Lackey

Department of Energy/HQ

Forrestal Building

1000 Independence Ave., SW

Washington, DC 20585

Dr. H. Coleman

Mr. S. Gronich

Mr. C. Mangold

Mr. M. Scheve

Mr. Frank Wilkins

Department of Energy/SAN

1333 Broadway

Oakland, CA 94536

Mr. Robert Hughey

Mr. William Lambert

Department of Energy/SAO

1617 Cole Blvd.

Golden, CO 80401

Mr. Jerry Bellows

El Paso Electric

P.O. Box 982

El Paso, TX 79960

Mr. James E. Brown

Electric Power Research Institute

P.O. Box 10412

Palo Alto, CA 94303

Mr. Donald Augenstein

Entech, Incorporated

P.O. Box 612246

DFW Airport, TX 75261

Mr. Walter Hesse 
Georgia Institute of Technology

Atlanta, GA 30332

Mr. Tom Brown, Ph.D.

Jet Propulsion Laboratory

4800 Oak Grove Drive

Pasadena, CA 91109

Mr. William Owen

LaJet Energy Company

P.O. Box 3599

Abilene, TX 79604

Mr. Monte McGlaum

Luz Engineering Corp.

15720 Ventura Blvd.

Suite 504

Encino, CA 91436

Dr. David Kearney

Martin Marietta

P.O. Box 179

Denver, CO 80201

Mr. Tom Tracey

McDonnell Douglas Astronautics

Company

5301 Bolsa Ave.

Huntington Beach, CA 92647

Mr. Jim Rogan

Meridian Corporation

5113 Leesburg Pike

Suite 700

Falls Church, VA 22041

Mr. Dinesh Kumar

Mr. Jim Williamson

3M Corporation

3M Center Building, 207-1 W-08

St. Paul, MN 55101

Mr. David Hill

Mr. Burton A. Benson

NASA Lewis Research Center

21000 Brookpark Road

Cleveland, $\mathrm{OH} 44135$

Dr. Dennis Flood
NASA-Johnson Space Center

NASA Road One - EPS

Houston, TX 77058

Mr. William Simon

Pacific Gas and Electric Company

3400 Crow Canyon Rd.

San Ramon, CA 94583

Mr. Gerry Braun

Rockwell International

Energy Systems Group

8900 DeSoto Ave.

Canoga Park, CA 91304

Mr. Tom H. Springer

Sandia National Laboratories

Solar Department 8453

Livermore, CA 94550

Mr. A Skinrood

Dr. R. A. Rinne

Sandia National Laboratories

Solar Energy Department 6220

P.O. Box 5800

Albuquerque, NM 87185

Mr. John Otts

Mr. James Leonard

Dr. Donald Schuler

Science Applications, Inc.

10401 Rosselle Street

San Diego, CA 92121

Dr. Barry Butler

Solar Energy Industries Association

1717 Massachusetts Ave. NW No. 503

Washington, DC 20036

Mr. Carlo La Porta

Mr. David Goren

Mr. Hal Seilstad

Solar Kinetics, Inc.

P.O. Bux 47045

Dallas, TX 75247

Mr. Gus Hutchison 
Southern California Edison 2244 Walnut Grove Ave.

Rosemead, CA 91770

Mr. Joe Reeves

University of Arizona

Dept. of Mechanical and Aerospace

Engineering

Tucson, AZ 85721

Dr. Kumar Ramohalli
University of Arizona

Dept. of Electrical Engineering

Tucson, AZ 85721

Dr. Roger Jones

Solar Energy Research Institute

Mr. Bim Gupta

Dr. Dave Johnson

Dr. Larry Shannon

Mr. Al Lewandowski

Dr. L. M. Murphy 


\begin{tabular}{|c|c|c|}
\hline $\begin{array}{l}\text { Document Control } \\
\text { Page }\end{array}$ & \begin{tabular}{|l|l|} 
1. SERI Report No. & 2. NTIS Accession No \\
SERI/TR-253-2338 & \\
\end{tabular} & 3 Recipient's Accession No. \\
\hline \multirow{2}{*}{\multicolumn{2}{|c|}{$\begin{array}{l}\text { 4. Title and Subtitle } \\
\text { Stability and Initial Imperfection Considerations } \\
\text { for Stretched-Membrane Reflector Modules }\end{array}$}} & $\begin{array}{l}\text { 5. Publication Date } \\
\text { October } 1986\end{array}$ \\
\hline & & 6. \\
\hline \multicolumn{2}{|c|}{$\begin{array}{l}\text { 7. Author(s) } \\
\text { L. M. Murphy, D. V. Sallis, D. Simms }\end{array}$} & 8. Performing Organization Rept. No \\
\hline \multirow{2}{*}{\multicolumn{2}{|c|}{$\begin{array}{l}\text { 9. Performing Organization Name and Address } \\
\text { Solar Energy Research Institute } \\
\text { A Division of Midwest Research Institute } \\
1617 \text { Cole Boulevard } \\
\text { Golden, Colorado } 80401-3393\end{array}$}} & $\begin{array}{c}\text { 10. Froject/Task/Work Unit No. } \\
5111.31\end{array}$ \\
\hline & & $\begin{array}{l}\text { 11. Contract (C) or Grant (G) No. } \\
\text { (C) } \\
\text { (G) }\end{array}$ \\
\hline \multirow{2}{*}{\multicolumn{2}{|c|}{ 12. Sponsoring Organization Name and Address }} & $\begin{array}{l}\text { 13. Type of Report \& Period Covered } \\
\text { Technical Report }\end{array}$ \\
\hline & & 14. \\
\hline \multicolumn{3}{|l|}{ 15. Supplementary Notes } \\
\hline \multicolumn{3}{|c|}{$\begin{array}{l}\text { This report extends the work of several previous reports that present the background } \\
\text { leading to the development of stretched-membrane modules and analysis methods to study } \\
\text { the structural response of the stretched-membrane module. Specifically, this report presents } \\
\text { and discusses the design implications based on our analysis of single- or double-membrane } \\
\text { concepts, and the amplification of initial imperfections and deflections caused by loading, } \\
\text { which results from stability considerations. } \\
\text { In this document, we present analysis results for both single- and double-membrane } \\
\text { concepts corresponding to a range of design and loading conditions. Further, we show that } \\
\text { stretched-membrane/frame combinations respond quite differently to external loads than can } \\
\text { be inferred by studying the decoupled frame and membrane independently. Thus the coupled } \\
\text { membrane/frame problem should be considered to assure an accurate description of its } \\
\text { response. For idealized configurations and loadings, we discuss the relative merits of various } \\
\text { design features for both of these designs. In addition, we studied the structural stability (i.e., } \\
\text { the tendency of structural deformation to grow with little increase in applied load) of the } \\
\text { tensioned-membrane, compressed-frame combination. Moreover, we demonstrate how } \\
\text { stability considerations are important in determining the amplification of both initial } \\
\text { displacement imperfection and the deformations caused by wind and weight loading on the } \\
\text { structure. }\end{array}$} \\
\hline \multicolumn{3}{|c|}{$\begin{array}{l}\text { 17. Document Analysis } \\
\text { a. Descriptors Deformation; Heliostats; Mechanical Structures; Membranes }\end{array}$} \\
\hline \multicolumn{3}{|c|}{ c. UC Categories $62,62 \mathrm{c}$} \\
\hline \multirow{2}{*}{\multicolumn{2}{|c|}{$\begin{array}{l}\text { 18. Avaitability Statement } \\
\text { National Technical Information Service } \\
\text { U.S. Department of Commerce } \\
5285 \text { Port Royal Road } \\
\text { Springfield, Virginia } 22161\end{array}$}} & $\begin{array}{c}19 \text { No. of Pages } \\
70\end{array}$ \\
\hline & & $\begin{array}{r}\text { 20. Price } \\
\mathrm{AO4}\end{array}$ \\
\hline
\end{tabular}

